\title{
Highly Diastereoselective Thioglycosylation of Functionalized Peracetylated Glycosides Catalyzed by $\mathrm{MoO}_{2} \mathrm{Cl}_{2}$
}

\author{
Shiue-Shien Weng, Yow-Dzer Lin, and Chien-Tien Chen* \\ Department of Chemistry, National Taiwan Normal University, Taipei, Taiwan \\ \#88, Sec. 4, Ding-jou Road, Taipei, Taiwan 11650 \\ e-mail: $\underline{\text { chefv043@ntnu.edu.tw }}$
}

Representative experimental procedures, spectral data (51 pages)

\section{SUPPORTING INFORMATION}

\section{Table of contents}

General experimental methods

Materials and preparation of known starting materials by literature methods $-\mathrm{S} 2-\mathrm{S} 3$

Representative procedure for the thioglycosylation $-\mathrm{S} 3$

Effects of oxometallic species on catalytic thioglycosylation of penta- $O$-acetyl- $\beta$-D-glucose with thiophenolS4

Analytical data for $S$-substituted-1-thio- $\beta$-D-glucopyranoside $\mathbf{2 a - 2 n}$ $-\mathrm{S} 5-\mathrm{S} 10$

Analytical data for phenyl-1-thio- $\beta$-glycosides 3-11 $-\mathrm{S} 10-\mathrm{S} 13$

Synthesis and analytical data for $p$-tolyl-1-thio- $\beta$-D-glucopyranoside-12' -S14

Synthesis and analytical data for 4-methylphenyl-4,6- $O$-benzylidene-1-thio- $\beta$-D-glucopyrano -side-12 $-\mathrm{S} 15$

Preparation of thioglycosidic disaccharide-15 S15-18

References -S18-S19

${ }^{1} \mathrm{H}$ and ${ }^{13} \mathrm{C}$ NMR spectra for $S$-substituted-1-thio- $\beta$-D-glucopyranosides $\mathbf{2 a - 2 n}$ - S20-S33

${ }^{1} \mathrm{H}$ and ${ }^{13} \mathrm{C}$ NMR spectra for phenyl-1-thio- $\beta$-glycosides 3-14-

${ }^{1} \mathrm{H},{ }^{13} \mathrm{C}, \mathrm{COSY}$, DEPT-90, DEPT-135, HMQC, and HMBC NMR spectra for thioglycosidic disaccharide-15 $-S 47-S 51$ 
General experimental methods. ${ }^{1} \mathrm{H}-\mathrm{NMR}$ and ${ }^{13} \mathrm{C}-\mathrm{NMR}$ were recorded on Varian Gemini-2000

(400 MHz ${ }^{1} \mathrm{H}, 100 \mathrm{MHz}{ }^{13} \mathrm{C}$ ) spectrometers in deuterochloroform with tetramethylsilane (TMS) or chloroform as an internal reference unless otherwise stated. Chemical shifts are reported in ppm ( $\delta)$, coupling constants, $J$, are reported in Hz. Mass spectra were recorded on a Finnigan TCQ-700 spectrometer with an ionization voltage of $70 \mathrm{eV}$ unless otherwise stated. Electrospray ionization (ESI) mass spectra were recorded on a Finnigan MAT-95S spectrometer. Data are reported in the form $\mathrm{m} / \mathrm{e}$ (intensity relative to base $=100 \%$ ). Elemental analysis data were obtained on a Heraeus CHN-OS Rapid analyzer. Optical rotations were recorded on a JASCO DIP-1000 digital polarimeter and are reported as follows: $(c=g / 100 \mathrm{ml}$, solvent). Analytical TLC was performed on Merck silica gel plates with QF-254 indicator. Visualization was accomplished with UV light or with phosphomolybdic acid (PMA) and $\mathrm{KMnO}_{4}$ staining agents. Column (flash) chromatography was performed by using 32-63 $\mu \mathrm{m}$ silica gel. Solvents for extraction and chromatography were reagent grade. Optical rotations were recorded on a JASCO DIP-1000 digital polarimeter and are reported as follows: $[\alpha]_{\mathrm{D}}^{\mathrm{T}}(\mathrm{c}=\mathrm{g} / 100 \mathrm{~mL}$, solvent $) . \mathrm{CH}_{2} \mathrm{Cl}_{2}, \mathrm{Cl}\left(\mathrm{CH}_{2}\right)_{2} \mathrm{Cl}, \mathrm{CHCl}_{3}, \mathrm{CCl}_{4}$, and $N, N$-dimethylformamide (DMF) were dried over $\mathrm{CaH}_{2}$ under nitrogen atmosphere and distilled before use. THF, $\mathrm{Et}_{2} \mathrm{O}, 1$,4-dioxane, and toluene were dried over benzophenone-ketyl intermediate under nitrogen atmosphere and distilled before use.. $\mathrm{CH}_{3} \mathrm{CN}$ was dried over $\mathrm{Mg}$ turnings under argon atmosphere and distilled before use. All reactions were performed with oven-dried $\left(120{ }^{\circ} \mathrm{C}\right)$ or flame-dried glassware under an atmosphere of dry argon.

\section{Materials and preparation of known starting materials by literature methods}

D-Glucose, D-galactose, D-mannose, D-xylose, D-lactose, D-maltose, L-arabinose, and 2,3,5tri- $O$-acetyl- $\beta$-D-ribofuranoside were purchased from ACROS. Methyltrioxorhenium(VII) $\left(\mathrm{CH}_{3} \mathrm{ReO}_{3}\right)$, chromyl(VI) chloride $\left(\mathrm{CrO}_{2} \mathrm{Cl}_{2}\right)$, bismuth(III) oxychloride (BiOCl), molybdenum(VI) dioxodichlororide $\left(\mathrm{MoO}_{2} \mathrm{Cl}_{2}\right)$, molybdenum acetylacetonate $\left(\mathrm{MoO}_{2}(\mathrm{acac})_{2}\right)$, oxomolybdenum (VI) tetrachloride $\left(\mathrm{MoOCl}_{4}\right)$, molybdenum(V) chloride $\left(\mathrm{MoCl}_{5}\right)$, hafnium(IV) oxychloride $\left(\mathrm{HfOCl}_{2}\right)$, and 
vanadyl(IV) acetylacetonate $\left(\mathrm{VO}(\mathrm{acac})_{2}\right)$ were purchased from Aldrich. The $\mathrm{VOX}_{2}$ series of compounds (brand name as Clip-all@ series, US patent \# 6,541,659 B1, 2003) and $\mathrm{TiOX}_{2}$ are now available directly from the institution (e-mail: chefv043@ntnu.edu.tw). Thiophenol, 4-methylthiophenol, 4-methoxythiopenol, 2-methoxythiophenol, 1,3-propanedithiol, ethanethiol, cyclohexyl mercaptan, tert-butylmercaptan, benzylmercaptan, and 2-mercaptoethanol were purchased from ACROS. Ther are freshly distilled from $\mathrm{CaSO}_{4}$ before use. 11-mercaptoundecanoic acid (re-crystallized from ether), 4-methyl-thiophenol, 2-naphthalenthiol, and 4-chlorothiophenol (re-crystallized from benzene) were purchased from ACROS. Penta- $O$-acetyl- $\beta$-D-glucose, ${ }^{1}$ penta- $O$-acetyl- $\beta$-D-galactose, ${ }^{2}$ penta- $O$-acetyl- $\beta$-D-mannose, ${ }^{3}$ tetra- $O$-acetyl- $\beta$-D-xylose,${ }^{4}$ tetra- $O$ acetyl- $\alpha$-L-arabinose, ${ }^{5}$ hepta- $O$-acetyl- $\beta$-D-lactose, hepta- $O$-acetyl- $\beta$-D-maltose, ${ }^{6}$ 2-(tert-butyldimethylsiloxy)ethylmercaptan, ${ }^{7}$ and methyl 11-mercaptoundecanoate ${ }^{8}$ were prepared by following literature methods.

\section{Representative Procedure for the catalytic thioglycosylation}

In a 25-mL, two-necked, round-bottomed flask equipped with an addition funnel was placed $\mathrm{MoO}_{2} \mathrm{Cl}_{2}$ (5.9 mg, $\left.0.03 \mathrm{mmol}, 3 \mathrm{~mol} \%\right)$ followed by addition of anhydrous $\mathrm{CH}_{2} \mathrm{Cl}_{2}(1 \mathrm{~mL})$. To this solution was slowly added a solution of a per-acetylated sugar $(1 \mathrm{mmol})$ in $\mathrm{CH}_{2} \mathrm{Cl}_{2}(3 \mathrm{~mL})$ at ambient temperature via the addition funnel. After $10 \mathrm{~min}$, a solution of thiol (1.3-1.5 equiv or 3.0 equiv for $t$-butanethiol) in $\mathrm{CH}_{2} \mathrm{Cl}_{2}(1 \mathrm{~mL})$ was slowly added to the reaction mixture. The reaction solution immediately turned deep blue or green and gradually changed to yellowish brown in 10 min. After completion of the reaction as evidenced by the solution color (deep brown) and by TLC analysis, the reaction mixture was quenched with cold saturated aqueous $\mathrm{NaHCO}_{3}(5 \mathrm{~mL})$. The organic layer was separated and washed with brine, dried $\left(\mathrm{MgSO}_{4}\right)$, filtered, and evaporated. The crude residue was purified by column chromatography on silica gel. The product was characterized by routine spectroscopic methods. 
Effect of oxometallic species on catalytic thioglycosylation of penta- $O$-acetyl- $\beta$-D-glucose with

thiophenol.

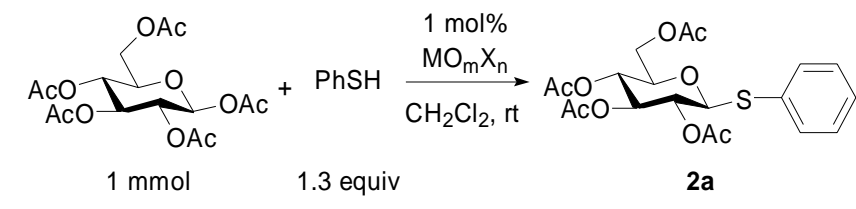

\begin{tabular}{cccc}
\hline entry $^{a}$ & $\mathrm{MO}_{\mathrm{m}} \mathrm{X}_{\mathrm{n}}$ & time (h) & yield, $^{a} \%$ \\
\hline 1 & $\mathrm{VO}(\mathrm{OTf})_{2}$ & 24 & $32\left(67^{c}\right)$
\end{tabular}

$\begin{array}{llll}2 & \mathrm{VO}(\mathrm{OTs})_{2}{ }^{b} & 24 & 16\end{array}$

\begin{tabular}{cccc}
3 & $\mathrm{VOCl}_{2}$ & 24 & 6 \\
4 & $\mathrm{VO}(\mathrm{acac})_{2}$ & 24 & 7 \\
5 & $\mathrm{BiOCl}$ & 24 & none \\
\hline 6 & $\mathrm{CrO}_{2} \mathrm{Cl}_{2}$ & 24 & none
\end{tabular}

7

8

9

10

11

12

13

$\mathrm{ZrO}(\mathrm{OTf})_{2}$

24

none

$\mathrm{CH}_{3} \mathrm{ReO}_{3}$

24

7

$\mathrm{MoO}_{2}(\mathrm{acac})_{2}$

24

trace

$\mathrm{MoO}_{2} \mathrm{Cl}_{2}$

14

$88\left(93^{c}\right)$

14

$\mathrm{HfOCl}_{2}$

14

$58 \%$

16

$\mathrm{MoCl}_{5}$

24

none

17

$$
\mathrm{Cu}(\mathrm{OTf})_{2}
$$

24

none

18

$$
(\mathrm{n}-\mathrm{Bu})_{2} \mathrm{SnO}
$$

24

none

none

none

none

\footnotetext{
${ }^{a}$ Isolated yields and no $\alpha$-anomer was observed. ${ }^{b}$ Vanadyl $p$-toluenesulfonate. ${ }^{c}$ Three mol \% of catalyst was used.
} 


\section{Analytical data for $S$-substituted -1-thio- $\beta$-D-glucopyranosides}

Phenyl-2,3,4,6-tetra- $O$-acetyl-1-thio- $\beta$-D-glucopyranoside $2 a^{9}$

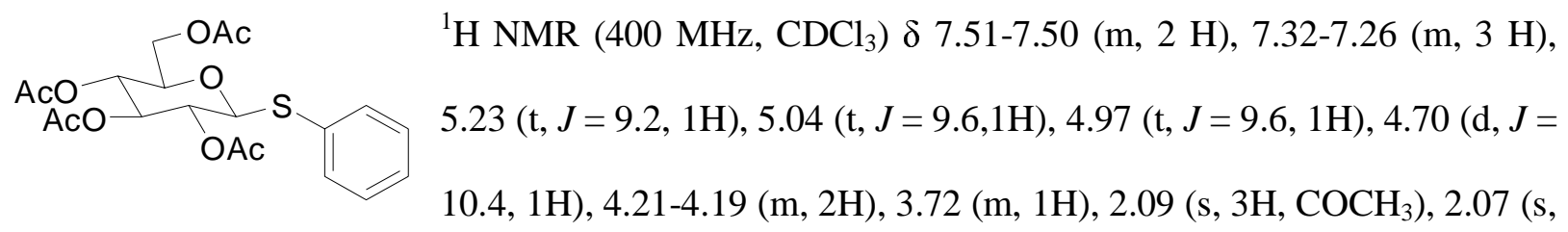

$\left.3 \mathrm{H}, \mathrm{COCH}_{3}\right), 2.01\left(\mathrm{~s}, 3 \mathrm{H}, \mathrm{COCH}_{3}\right), 1.98\left(\mathrm{~s}, 3 \mathrm{H}, \mathrm{COCH}_{3}\right) ;{ }^{13} \mathrm{C} \mathrm{NMR}\left(100 \mathrm{MHz}, \mathrm{CDCl}_{3}\right) \delta 170.51$, $170.12,169.34,169.2,133.10,131.61,128.9,128.4,85.7,75.8,73.9,69.9,68.2,62.1,20.70,20.7$, 20.6, 20.5; M.W. $\left(\mathrm{C}_{20} \mathrm{H}_{24} \mathrm{O} 9 \mathrm{~S}\right)$ 440.46; ESI-MS m/z $463\left(\mathrm{M}+\mathrm{Na}^{+}, 71\right) ; \mathrm{mp} 118-120{ }^{\circ} \mathrm{C} ;[\alpha]_{\mathrm{D}}^{23}-22.7$ (c 1.0, $\mathrm{CHCl}_{3}$ ); TLC $\mathrm{R}_{f} 0.35$ (Ethyl acetate/hexane, 1/ 2).

$p$-Tolyl-2,3,4,6-tetra- $O$-acetyl-1-thio- $\beta$-D-glucopyranoside $2 b^{10}$

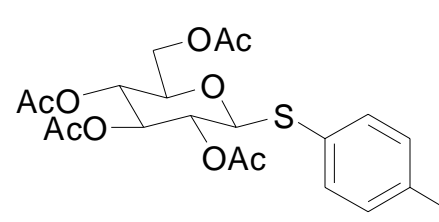

$$
\begin{aligned}
& { }^{1} \mathrm{H} \text { NMR }\left(400 \mathrm{MHz}, \mathrm{CDCl}_{3}\right) \delta 7.39(\mathrm{~d}, J=8.1,2 \mathrm{H}), 7.39(\mathrm{~d}, J=7.9, \\
& 2 \mathrm{H}), 5.21(\mathrm{t}, J=9.4,1 \mathrm{H}), 5.02(\mathrm{t}, J=9.7,1 \mathrm{H}), 4.94(\mathrm{t}, J=10.1,1 \mathrm{H}), \\
& 4.63(\mathrm{~d}, J=10.0,1 \mathrm{H}), 4.21-4.18(\mathrm{~m}, 2 \mathrm{H}), 3.70(\mathrm{ddd}, J=10.1,4.7,2.7,
\end{aligned}
$$

1H), $2.35(\mathrm{~s}, 3 \mathrm{H}), 2.09\left(\mathrm{~s}, 3 \mathrm{H}, \mathrm{COCH}_{3}\right), 2.08\left(\mathrm{~s}, 3 \mathrm{H}, \mathrm{COCH}_{3}\right), 2.01\left(\mathrm{~s}, 3 \mathrm{H}, \mathrm{COCH}_{3}\right), 1.98(\mathrm{~s}, 3 \mathrm{H}$, $\left.\mathrm{COCH}_{3}\right) ;{ }^{13} \mathrm{C} \mathrm{NMR}\left(100 \mathrm{MHz}, \mathrm{CDCl}_{3}\right) \delta 170.6,170.2,169.4,169.2,138.8,129.7,127.6,85.8,75.8$, 74.0, 69.9, 68.2, 62.1, 21.2, 20.7, 20.7,20.6, 20.6; M.W. $\left(\mathrm{C}_{21} \mathrm{H}_{26} \mathrm{O}_{9} \mathrm{~S}\right)$ 454.49; ESI-MS m/z 477 $\left(\mathrm{M}+\mathrm{Na}^{+}, 27\right), 478(12) ; \operatorname{mp} 114-116{ }^{\circ} \mathrm{C} ;[\alpha]_{\mathrm{D}}^{24}-18.2\left(c \quad 1.2, \mathrm{CHCl}_{3}\right) ;$ TLC $\mathrm{R}_{f} 0.32$ (Ethyl acetate/hexane, 1/2).

\section{4-Chlorophenyl-2, 3,4,6-tetra- $O$-acetyl-1-thio- $\beta$-D-glucopyranoside $2 \mathrm{c}^{11}$}

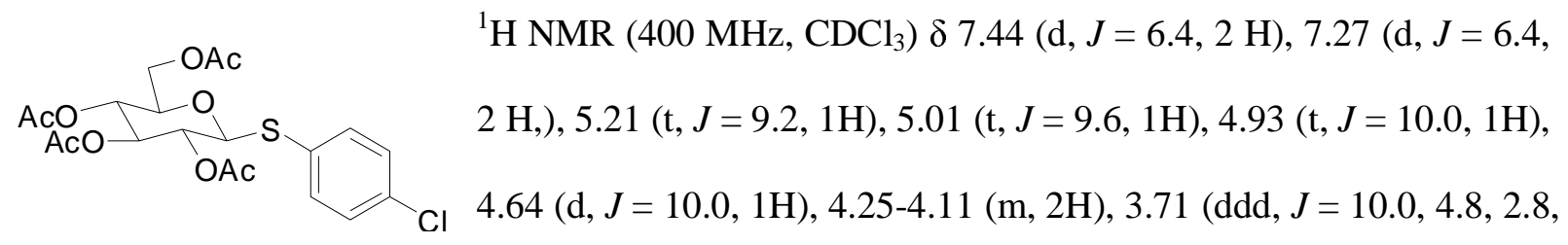

$1 \mathrm{H}), 2.07\left(\mathrm{~s}, 3 \mathrm{H}, \mathrm{COCH}_{3}\right), 2.06\left(\mathrm{~s}, 3 \mathrm{H}, \mathrm{COCH}_{3}\right), 2.00\left(\mathrm{~s}, 3 \mathrm{H}, \mathrm{COCH}_{3}\right), 1.97\left(\mathrm{~s}, 3 \mathrm{H}, \mathrm{COCH}_{3}\right) ;{ }^{13} \mathrm{C}$ NMR (100 MHz, $\left.\mathrm{CDCl}_{3}\right) \delta 170.4,170.1,169.3,169.1,134.9,129.4,85.2,75.8,73.8,69.8,68.1$, 
62.0, 20.7, 20.6; M.W. $\left(\mathrm{C}_{20} \mathrm{H}_{23} \mathrm{ClO}_{9} \mathrm{~S}\right)$ 474.19; ESI-MS m/z 497 (M+Na $\left.{ }^{+}, 100\right), 498$ (67); mp $112-113{ }^{\circ} \mathrm{C} ;[\alpha]_{\mathrm{D}}^{26}-26.4\left(c 2.0, \mathrm{CHCl}_{3}\right) ; \mathrm{TLC} \mathrm{R}_{f} 0.36$ (Ethyl acetate/hexane, $\left.1 / 2\right)$.

4-Methoxyphenyl-2,3,4,6-tetra- $O$-acetyl-1-thio- $\beta$-D-glucopyranoside $2 \mathrm{~d}^{12}$

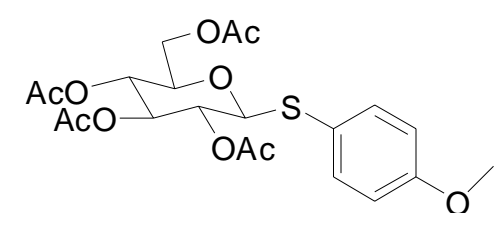

${ }^{1} \mathrm{H}$ NMR $\left(400 \mathrm{MHz}, \mathrm{CDCl}_{3}\right) \delta 7.49(\mathrm{~d}, J=10.8,2 \mathrm{H}), 6.84(\mathrm{~d}, J=$ $10.8,2 \mathrm{H}), 5.17(\mathrm{t}, J=9.2,1 \mathrm{H}), 4.97(\mathrm{t}, J=10.0,1 \mathrm{H}), 4.87(\mathrm{t}, J=$ $9.6,1 \mathrm{H}), 4.53(\mathrm{~d}, J=10.0,1 \mathrm{H}), 4.19(\mathrm{dd}, J=12.0,4.4,1 \mathrm{H}), 4.15$

$(\mathrm{dd}, J=12.3,2.2,1 \mathrm{H}), 3.80\left(\mathrm{~s}, 3 \mathrm{H}, \mathrm{OCH}_{3}\right), 3.66(\mathrm{ddd}, J=7.2,4.0,3.2,1 \mathrm{H}), 2.08\left(\mathrm{~s}, 3 \mathrm{H}, \mathrm{COCH}_{3}\right)$, $2.05\left(\mathrm{~s}, 3 \mathrm{H}, \mathrm{COCH}_{3}\right), 1.99\left(\mathrm{~s}, 3 \mathrm{H}, \mathrm{COCH}_{3}\right), 1.96\left(\mathrm{~s}, 3 \mathrm{H}, \mathrm{COCH}_{3}\right) ;{ }^{13} \mathrm{C} \mathrm{NMR}\left(100 \mathrm{MHz}, \mathrm{CDCl}_{3}\right) \delta$ $170.5,170.1,169.3,169.2,160.4,136.5,120.8,114.4,85.6,75.7,74.0,69.9,68.2,62.0,55.3,20.7$ 20.7, 20.5, 20.5; M.W. $\left(\mathrm{C}_{21} \mathrm{H}_{26} \mathrm{O}_{10} \mathrm{~S}\right)$ 470.49; ESI-MS m/z $493\left(\mathrm{M}+\mathrm{Na}^{+}, 30\right), 494$ (18); mp 92-93 ${ }^{\circ} \mathrm{C}$; $[\alpha]_{\mathrm{D}}^{26}-24.3\left(c\right.$ 2.0, $\left.\mathrm{CHCl}_{3}\right) ;$ TLC $\mathrm{R}_{f} 0.31$ (Ethyl acetate/hexane, 1/ 2).

2-Naphthyl-2,3,4,6-tetra- $O$-acetyl-1-thio- $\beta$-D-glucopyranoside $2 \mathrm{e}^{13}$

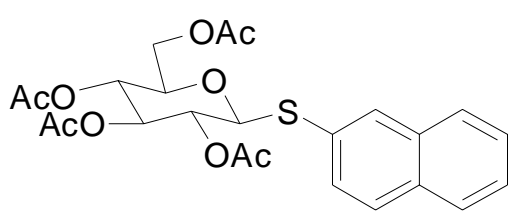

${ }^{1} \mathrm{H}$ NMR (400 MHz, $\left.\mathrm{CDCl}_{3}\right) \delta 7.99(\mathrm{~s}, 1 \mathrm{H}), 7.83-7.77$ (m, 3H), 7.57-7.46 (m, 3H), $5.24(\mathrm{t}, J=9.7,1 \mathrm{H}), 5.07-4.99(\mathrm{~m}, 21 \mathrm{H})$, $4.78(\mathrm{~d}, J=10.0,1 \mathrm{H}), 4.23(\mathrm{dd}, J=12.3,5.1,1 \mathrm{H}), 4.18(\mathrm{dd}, J=$

12.3, 2.4, 1H, $), 3.72(\mathrm{ddd}, J=7.4,4.9,2.4,1 \mathrm{H}), 2.11\left(\mathrm{~s}, 3 \mathrm{H}, \mathrm{COCH}_{3}\right), 2.02\left(\mathrm{~s}, 3 \mathrm{H}, \mathrm{COCH}_{3}\right), 2.00(\mathrm{~s}$, $\left.3 \mathrm{H}, \mathrm{COCH}_{3}\right), 1.98\left(\mathrm{~s}, 3 \mathrm{H}, \mathrm{COCH}_{3}\right) ;{ }^{13} \mathrm{C} \mathrm{NMR}\left(100 \mathrm{MHz}, \mathrm{CDCl}_{3}\right) \delta 170.5,170.1,169.3,169.2$, $133.4,132.9,132.9,132.7,130.2,128.7,128.4,127.7,126.7,126.6,85.8,75.9,73.9,70.0,68.2$,

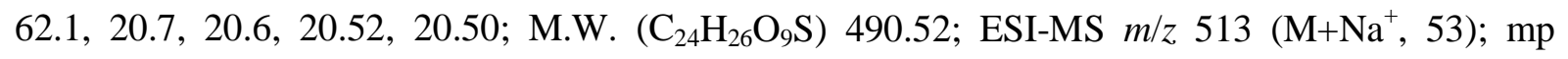
108-109 ${ }^{\circ} \mathrm{C} ;[\alpha]_{\mathrm{D}}^{24}-16.2\left(c 1.0, \mathrm{CHCl}_{3}\right)$; TLC $\mathrm{R}_{f} 0.36$ (Ethyl acetate/hexane, $\left.1 / 2\right)$.

\section{2-Methoxyphenyl-2,3,4,6-tetra- $O$-acetyl-1-thio- $\beta$-D-glucopyranoside 2 f}

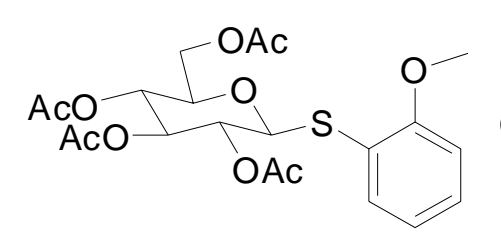

${ }^{1} \mathrm{H}$ NMR $\left(400 \mathrm{MHz}, \mathrm{CDCl}_{3}\right) \delta 7.47(\mathrm{~d}, J=8.8,1 \mathrm{H}), 7.29(\mathrm{~m}, 1 \mathrm{H})$, 6.91-6.86 (m, $2 \mathrm{H}), 5.22(\mathrm{t}, J=9.4,1 \mathrm{H}), 5.05(\mathrm{t}, J=9.8,1 \mathrm{H}), 4.99$ 
$(\mathrm{t}, J=9.2,1 \mathrm{H}),, 4.79(\mathrm{~d}, J=10.0,1 \mathrm{H}), 4.19(\mathrm{dd}, J=12.2,5.2,1 \mathrm{H}), 4.15(\mathrm{dd}, J=12.2,2.4,1 \mathrm{H})$, $\left.3.84\left(\mathrm{~s}, 3 \mathrm{H}, \mathrm{OCH}_{3}\right)\right), 3.75(\mathrm{ddd}, J=7.0,3.8,2.8,1 \mathrm{H}), 2.05\left(\mathrm{~s}, 3 \mathrm{H}, \mathrm{COCH}_{3}\right), 2.04\left(\mathrm{~s}, 3 \mathrm{H}, \mathrm{COCH}_{3}\right)$, $2.01\left(\mathrm{~s}, 3 \mathrm{H}, \mathrm{COCH}_{3}\right), 1.98\left(\mathrm{~s}, 3 \mathrm{H}, \mathrm{COCH}_{3}\right) ;{ }^{13} \mathrm{C} \mathrm{NMR}\left(100 \mathrm{MHz}, \mathrm{CDCl}_{3}\right) \delta 170.5,170.2,169.4$ $169.3,158.5,133.6,129.6,121.0,120.0,111.0,84.3,75.7,74.1,70.1,68.3,62.2,55.7,20.7,20.6$ 20.5; M.W. $\left(\mathrm{C}_{21} \mathrm{H}_{26} \mathrm{O}_{10} \mathrm{~S}\right)$ 470.49; ESI-MS m/z $493\left(\mathrm{M}+\mathrm{Na}^{+}, 15\right) ; \mathrm{mp} 88-90{ }^{\circ} \mathrm{C} ;[\alpha]_{\mathrm{D}}^{26}-19.6$ (c 2.0, $\mathrm{CHCl}_{3}$ ); TLC $\mathrm{R}_{f} 0.33$ (Ethyl acetate/hexane, 1/ 2); Anal. calcd for $\mathrm{C}_{21} \mathrm{H}_{26} \mathrm{O}_{10} \mathrm{~S}: \mathrm{C}, 53.61$; H, 5.57. Found: C, 53.67; H, 5.34.

$o$-Tolyl-2,3,4,6-tetra- $O$-acetyl-1-thio- $\beta$-D-glucopyranoside $2 \mathrm{~g}^{14}$

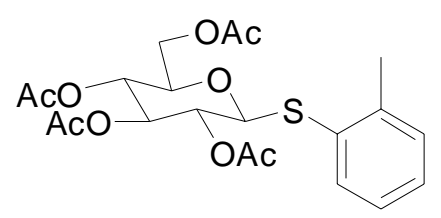

${ }^{1} \mathrm{H}$ NMR (400 MHz, $\left.\mathrm{CDCl}_{3}\right) \delta 7.49(\mathrm{~d}, J=7.7,1 \mathrm{H}), 7.21-7.13(\mathrm{~m}, 3$ $\mathrm{H}), 5.20(\mathrm{t}, J=12.0,1 \mathrm{H}), 5.08-5.01(\mathrm{~m}, 2 \mathrm{H}), 4.66(\mathrm{~d}, J=10.2,1 \mathrm{H})$, $4.21(\mathrm{dd}, J=12.3,5.6,1 \mathrm{H}), 4.12(\mathrm{dd}, J=12.3,2.2,1 \mathrm{H}), 3.67(\mathrm{~m}, 2 \mathrm{H})$, $2.38\left(\mathrm{~s}, 3 \mathrm{H}, \mathrm{CH}_{3}\right), 2.07$ (s, 3H, $\left.\mathrm{COCH}_{3}\right), 2.05\left(\mathrm{~s}, 3 \mathrm{H}, \mathrm{COCH}_{3}\right), 2.00\left(\mathrm{~s}, 3 \mathrm{H}, \mathrm{COCH}_{3}\right), 1.98(\mathrm{~s}, 3 \mathrm{H}$, $\left.\mathrm{COCH}_{3}\right) ;{ }^{13} \mathrm{C}$ NMR $\left(100 \mathrm{MHz}, \mathrm{CDCl}_{3}\right) \delta 170.4,170.1,169.3,169.2,140.4,131.8,130.3,128.4$, 126.5, 86.3, 75.7, 73.9, 70.1, 68.3, 62.2, 20.8, 20.61, 20.59, 20.49, 20.47; M.W. $\left(\mathrm{C}_{21} \mathrm{H}_{26} \mathrm{O}_{9} \mathrm{~S}\right) 454.49$; ESI MS $m / z 477\left(\mathrm{M}+\mathrm{Na}^{+}, 73\right) ; \mathrm{mp} 114-116{ }^{\circ} \mathrm{C} ;[\alpha]_{\mathrm{D}}^{26}-10.2\left(c 1.0, \mathrm{CHCl}_{3}\right) ;$ TLC $\mathrm{R}_{f} 0.33$ (Ethyl acetate/hexane, 1/2).

Ethyl-2,3,4,6-tetra- $O$-acetyl-1-thio- $\beta$-D-glucopyranoside $2 h^{15}$

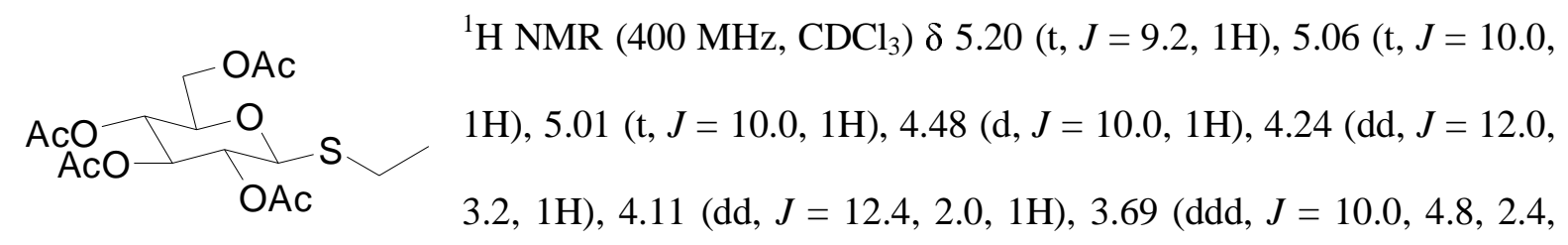
1H), 2.74-2.63 (m, 2H), $2.05\left(\mathrm{~s}, 3 \mathrm{H}, \mathrm{COCH}_{3}\right), 2.03\left(\mathrm{~s}, 3 \mathrm{H}, \mathrm{COCH}_{3}\right), 1.99\left(\mathrm{~s}, 3 \mathrm{H}, \mathrm{COCH}_{3}\right), 1.97(\mathrm{~s}$, $\left.3 \mathrm{H}, \mathrm{COCH}_{3}\right) ;{ }^{13} \mathrm{C} \mathrm{NMR}\left(\mathrm{CDCl}_{3}, 100 \mathrm{MHz}\right) \delta 170.6,170.1,169.3,169.3,83.5,75.8,73.9,69.8$, 68.3, 62.1, 24.1, 20.7, 20.53, 20.51, 14.8; M.W. $\left(\mathrm{C}_{16} \mathrm{H}_{24} \mathrm{O}_{9} \mathrm{~S}\right)$ 392.42; ESI-MS m/z 415 (M+Na $\left.{ }^{+}, 93\right)$; $\operatorname{mp} 82-83{ }^{\circ} \mathrm{C} ;[\alpha]_{\mathrm{D}}^{26}-25.6\left(c 1.0, \mathrm{CHCl}_{3}\right)$; $\mathrm{TLC} \mathrm{R}_{f} 0.36$ (Ethyl acetate/hexane, $1 / 2$ ). 
Benzyl-2,3,4,6-tetra- $O$-acetyl-1-thio- $\beta$-D-glucopyranoside $2 \mathrm{i}^{16}$

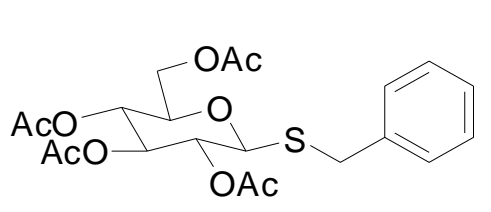

${ }^{1} \mathrm{H}$ NMR $\left(400 \mathrm{MHz}, \mathrm{CDCl}_{3}\right) \delta$ 7.33-7.27 (m, $\left.5 \mathrm{H}\right)$, 5.15-5.03 (m, $3 \mathrm{H}), 4.28(\mathrm{~d}, J=9.7,1 \mathrm{H}), 4.25(\mathrm{dd}, J=12.4,5.12,1 \mathrm{H}), 4.13(\mathrm{dd}$, $J=12.4,2.28,1 \mathrm{H}), 3.94(\mathrm{~d}, J=12.9,1 \mathrm{H}), 3.83(\mathrm{~d}, J=12.9,1 \mathrm{H})$,

3.59 (ddd, $J=9.7,5.0,2.3,1 \mathrm{H}), 2.11\left(\mathrm{~s}, 3 \mathrm{H}, \mathrm{COCH}_{3}\right), 2.06\left(\mathrm{~s}, 6 \mathrm{H}, 2 \times \mathrm{COCH}_{3}\right), 1.99^{\prime}(\mathrm{s}, 3 \mathrm{H}$, $\left.\mathrm{COCH}_{3}\right) ;{ }^{13} \mathrm{C} \mathrm{NMR}\left(100 \mathrm{MHz}, \mathrm{CDCl}_{3}\right) \delta 170.6,170.1,169.4,169.4,136.8,129.0,128.6,127.4$, 81.9, 75.8, 73.8, 69.6, 62.2, 33.7, 20.7, 20.6, 20.54, 20.52; M.W. $\left(\mathrm{C}_{21} \mathrm{H}_{26} \mathrm{O}_{9} \mathrm{~S}\right)$ 454.49; ESI-MS m/z $477\left(\mathrm{M}+\mathrm{Na}^{+}, 32\right), 478(18) ; \mathrm{mp} 98-99{ }^{\circ} \mathrm{C} ;[\alpha]_{\mathrm{D}}^{26}-88.2$ (c 1.0, $\mathrm{CHCl}_{3}$ ); TLC $\mathrm{R}_{f} 0.38$ (Ethyl acetate/hexane, 1/2).

\section{Cyclohexyl-2,3,4,6-tetra- $O$-acetyl-1-thio- $\beta$-D-glucopyranoside $2 \mathbf{j}^{17}$}

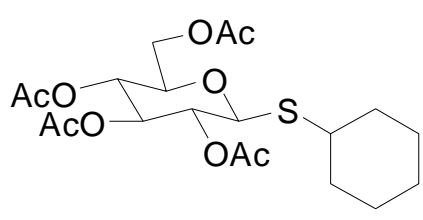

${ }^{1} \mathrm{H}$ NMR $\left(400 \mathrm{MHz}, \mathrm{CDCl}_{3}\right) \delta 5.21(\mathrm{t}, J=9.4,1 \mathrm{H}), 5.06(\mathrm{t}, J=9.9$, $1 \mathrm{H}), 4.98(\mathrm{t}, J=10.1,1 \mathrm{H}), 4.59(\mathrm{~d}, J=10.1,1 \mathrm{H}), 4.21(\mathrm{dd}, J=12.3$, $5.3,1 \mathrm{H}), 4.12(\mathrm{dd}, J=12.4,2.5,1 \mathrm{H}), 3.69(\mathrm{ddd}, J=10.0,5.4,2.5$, 1H), $2.89(\mathrm{~m}, 2 \mathrm{H}), 2.06\left(\mathrm{~s}, 3 \mathrm{H}, \mathrm{COCH}_{3}\right), 2.03\left(\mathrm{~s}, 3 \mathrm{H}, \mathrm{COCH}_{3}\right), 2.02\left(\mathrm{~s}, 3 \mathrm{H}, \mathrm{COCH}_{3}\right), 1.99(\mathrm{~s}, 3 \mathrm{H}$, $\left.\mathrm{COCH}_{3}\right), 1.98(\mathrm{~m}, 2 \mathrm{H}), 1.75(\mathrm{~m}, 2 \mathrm{H}), 1.43-1.21(\mathrm{~m}, 6 \mathrm{H}) ;{ }^{13} \mathrm{C} \mathrm{NMR}\left(\mathrm{CDCl}_{3}, 100 \mathrm{MHz}\right) \delta 170.6$ $170.2,169.4,169.3,83.2,75.7,74.0,70.3,68.5,62.3,43.9,34.2,33.9,26.0,25.9,25.6,20.72$, 20.67, 20.58, 20.56; M.W. $\left(\mathrm{C}_{20} \mathrm{H}_{30} \mathrm{O}_{9} \mathrm{~S}\right)$ 446.51; ESI-MS m/z $469\left(\mathrm{M}+\mathrm{Na}^{+}, 27\right)$; mp 120-122 ${ }^{\circ} \mathrm{C}$; $[\alpha]_{\mathrm{D}}^{27}-38.2\left(c 1.0, \mathrm{CHCl}_{3}\right) ;$ TLC $\mathrm{R}_{f} 0.40$ (Ethyl acetate/hexane, 1/ 2).

tert-Butyl-2,3,4,6-tetra- $O$-acetyl-1-thio- $\beta$-D-glucopyranoside $2 \mathbf{k}^{18}$

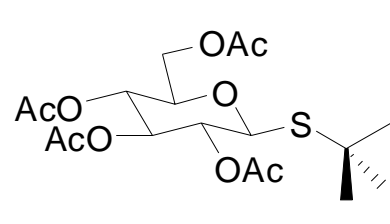

${ }^{1} \mathrm{H} \mathrm{NMR}\left(\mathrm{CDCl}_{3}, 400 \mathrm{MHz}\right) \delta 5.25(\mathrm{t}, J=9.3,1 \mathrm{H}), 5.03(\mathrm{t}, J=9.7,1 \mathrm{H})$, $4.94(\mathrm{t}, J=9.8,1 \mathrm{H}), 4.63(\mathrm{~d}, J=10.0,1 \mathrm{H}), 4.19(\mathrm{dd}, J=12.2,6.0,1 \mathrm{H})$ $4.10(\mathrm{dd}, J=12.2,2.2,1 \mathrm{H}), 3.71(\mathrm{ddd}, J=6.4,6.0,2.3,1 \mathrm{H}), 2.04(\mathrm{~s}$,

$\left.3 \mathrm{H}, \mathrm{COCH}_{3}\right), 2.03\left(\mathrm{~s}, 3 \mathrm{H}, \mathrm{COCH}_{3}\right), 2.02\left(\mathrm{~s}, 3 \mathrm{H}, \mathrm{COCH}_{3}\right), 2.00\left(\mathrm{~s}, 3 \mathrm{H}, \mathrm{COCH}_{3}\right), 1.36(\mathrm{~s}, 9 \mathrm{H}$, $\left.\mathrm{C}\left(\mathrm{CH}_{3}\right)_{3}\right) ;{ }^{13} \mathrm{C} \mathrm{NMR}\left(\mathrm{CDCl}_{3}, 100 \mathrm{MHz}\right) \delta 170.6,170.2,169.4,169.3,82.3,75.6,74.0,70.2,68.6$, 62.6, 44.3, 31.4, 20.7, 20.66, 20.58, 20.56; M.W. $\left(\mathrm{C}_{18} \mathrm{H}_{28} \mathrm{O}_{9} \mathrm{~S}\right)$ 420.47; ESI-MS m/z $443\left(\mathrm{M}^{2} \mathrm{Na}^{+}\right.$, 
13); mp 140-142 ${ }^{\circ} \mathrm{C} ;[\alpha]_{\mathrm{D}}^{27}-6.8\left(c 0.65, \mathrm{CHCl}_{3}\right)$; TLC $\mathrm{R}_{f} 0.23$ (Ethyl acetate/hexane, $\left.1 / 2\right)$.

3'-Mercapto-propyl 2, 3, 4, 6-tetra- $O$-acetyl-1-thio- $\beta$-D-glucopyranoside $21^{19}$

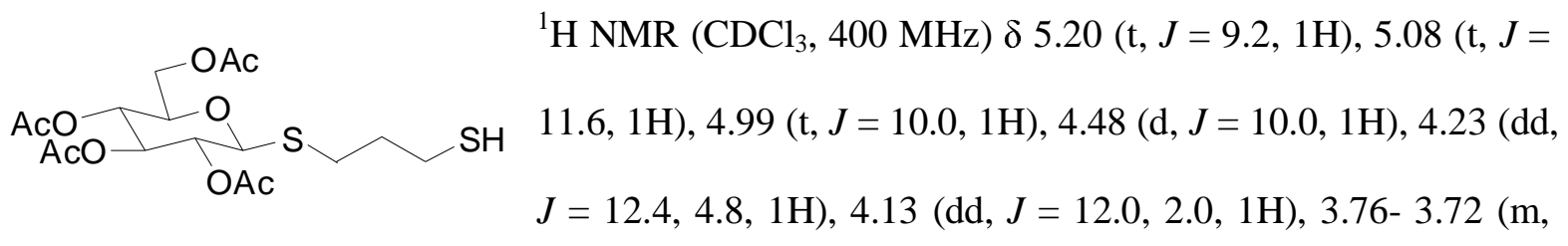

$1 \mathrm{H}), 2.81(\mathrm{dt}, J=13.0,6.8,1 \mathrm{H}), 2.71(\mathrm{dt}, J=12.8,6.81 \mathrm{H}), 2.61(\mathrm{td}, J=14.8,7.6,2 \mathrm{H}), 2.07(\mathrm{~s}, 3 \mathrm{H}$,

$\left.\mathrm{COCH}_{3}\right), 2.05\left(\mathrm{~s}, 3 \mathrm{H}, \mathrm{COCH}_{3}\right), 2.01\left(\mathrm{~s}, 3 \mathrm{H}, \mathrm{COCH}_{3}\right), 1.97\left(\mathrm{~s}, 3 \mathrm{H}, \mathrm{COCH}_{3}\right), 2.24-1.83(\mathrm{~m}, 4 \mathrm{H}), 1.89$

$(\mathrm{t}, J=7.2,2 \mathrm{H}), 1.34(\mathrm{t}, J=7.8,1 \mathrm{H}) ;{ }^{13} \mathrm{C} \mathrm{NMR}\left(\mathrm{CDCl}_{3}, 100 \mathrm{MHz}\right) \delta 170.5,170.1,169.33,169.32$

83.6, 75.9, 73.8, 69.8, 68.3, 62.1, 33.4, 28.2, 23.1, 20.69, 20.64, 20.53, 20.51; M.W. $\left(\mathrm{C}_{17} \mathrm{H}_{26} \mathrm{O}_{9} \mathrm{~S}_{2}\right)$

438.51; MS (EI, 70 eV) $438\left(\mathrm{M}^{+}, 39\right), 422(18), 421(100), 409(18) ;[\alpha]_{\mathrm{D}}^{28}-18.7\left(c\right.$ 1.0, $\left.\mathrm{CHCl}_{3}\right)$;

TLC $\mathrm{R}_{f} 0.42$ (Ethyl acetate/hexane, 1/ 2).

\section{1-Methoxycarbonyl undecanyl- 2, 3, 4, 6-tetra- $O$-acety l-1-thio- $\beta$-D-glucopyranoside $2 \mathrm{~m}^{20}$}

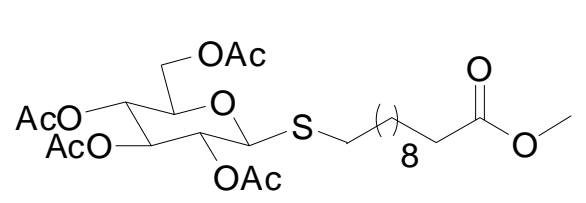

${ }^{1} \mathrm{H} \mathrm{NMR}\left(\mathrm{CDCl}_{3}, 400 \mathrm{MHz}\right) \delta .21(\mathrm{t}, J=9.2,1 \mathrm{H}), 5.07(\mathrm{t}, J=$ $10.0,1 \mathrm{H}), 5.02(\mathrm{dd}, J=11.6,11.2,1 \mathrm{H}), 4.47(\mathrm{~d}, J=10.4,1 \mathrm{H})$, $4.23(\mathrm{dd}, J=12.4,4.8,1 \mathrm{H}), 4.12(\mathrm{dd}, J=12.4,2.4,1 \mathrm{H}), 3.70$

$(\mathrm{ddd}, J=9.2,4.8,2.4,1 \mathrm{H}), 3.65\left(\mathrm{~s}, 3 \mathrm{H}, \mathrm{OCH}_{3}\right), 2.68-2.61(\mathrm{~m}, 2 \mathrm{H}), 2.29(\mathrm{t}, J=7.6,1 \mathrm{H}), 2.07(\mathrm{~s}, 3 \mathrm{H}$, $\left.\mathrm{COCH}_{3}\right), 2.05\left(\mathrm{~s}, 3 \mathrm{H}, \mathrm{COCH}_{3}\right), 2.01\left(\mathrm{~s}, 3 \mathrm{H}, \mathrm{COCH}_{3}\right), 1.99\left(\mathrm{~s}, 3 \mathrm{H}, \mathrm{COCH}_{3}\right), 1.65-1.52(\mathrm{~m}, 5 \mathrm{H})$, 1.36-1.26 (m, 13H); ${ }^{13} \mathrm{C} \mathrm{NMR}\left(\mathrm{CDCl}_{3}, 100 \mathrm{MHz}\right) \delta 174.3,170.6,170.2,169.4,169.3,83.6,75.8$, $73.9,69.9,68.3,62.2,51.4,34.1,30.0,29.6,29.4,29.3,29.2,29.1,28.7,24.9,20.7,20.6,20.5$;

M.W. $\left(\mathrm{C}_{26} \mathrm{H}_{42} \mathrm{O}_{11} \mathrm{~S}\right)$ 562.67; ESI-MS m/z $585\left(\mathrm{M}+\mathrm{Na}^{+}, 14\right)$; mp 54-56 ${ }^{\circ} \mathrm{C}$; $[\alpha]_{\mathrm{D}}^{27}-42.2\left(c\right.$ 1.0, $\left.\mathrm{CHCl}_{3}\right)$; TLC $\mathrm{R}_{f} 0.38$ (Ethyl acetate/hexane, 1/ 3).

2'-tert-Butyl dimethyl silyl 2, 3, 4, 6-tetra- $O$-acetyl-1-thio- $\beta$-D-glucopyranoside 2 n

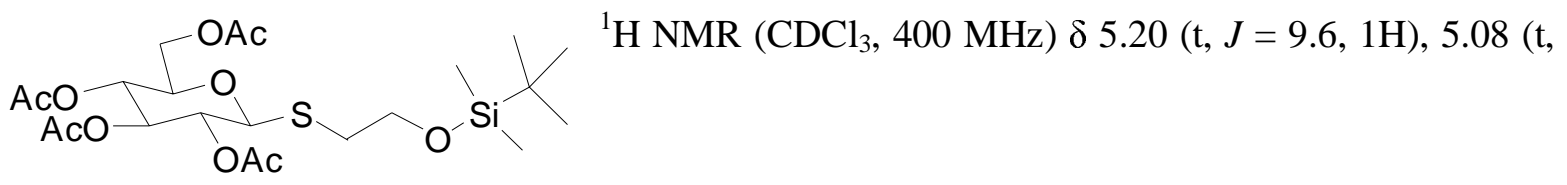


$J=9.6,1 \mathrm{H}), 5.01(\mathrm{t}, J=10.0,1 \mathrm{H}), 4.58(\mathrm{~d}, J=10.0,1 \mathrm{H}), 4.24(\mathrm{dd}, J=12.4,4.8,1 \mathrm{H}), 4.13(\mathrm{dd}, J=$ $12.4,2.4,1 \mathrm{H}), 3.85-3.73(\mathrm{~m}, 2 \mathrm{H}), 3.69(\mathrm{ddd}, J=7.2,4.8,2.4,1 \mathrm{H}), 2.88(\mathrm{dt}, J=14.0,7.6,1 \mathrm{H}), 2.71$ $(\mathrm{dt}, J=13.6,6.0,1 \mathrm{H}), 2.08\left(\mathrm{~s}, 3 \mathrm{H}, \mathrm{COCH}_{3}\right), 2.05\left(\mathrm{~s}, 3 \mathrm{H}, \mathrm{COCH}_{3}\right), 2.02\left(\mathrm{~s}, 3 \mathrm{H}, \mathrm{COCH}_{3}\right), 1.99$ (s, $\left.3 \mathrm{H}, \mathrm{COCH}_{3}\right), 0.90\left(\mathrm{~s}, 9 \mathrm{H}, \mathrm{C}\left(\mathrm{CH}_{3}\right)_{3}\right), 0.07\left(\mathrm{~s}, 6 \mathrm{H}, 2 \times \mathrm{CH}_{3}\right) ;{ }^{13} \mathrm{C} \mathrm{NMR}\left(\mathrm{CDCl}_{3}, 100 \mathrm{MHz}\right) \delta 170.6$, $170.1,169.4,169.3,83.6,75.9,73.8,70.0,68.3,63.3,62.1,32.3,25.8,20.68,20.65,20.55,20.53$, 18.2, -5.31, -5.32; M.W. $\left(\mathrm{C}_{22} \mathrm{H}_{38} \mathrm{O}_{10} \mathrm{SSi}\right)$ 522.68; ESI-MS m/z $545\left(\mathrm{M}+\mathrm{Na}^{+}, 45\right)$; colorless oil; $[\alpha]_{\mathrm{D}}^{23}$ -18.2 ( $c$ 1.0, $\mathrm{CHCl}_{3}$ ); TLC $\mathrm{R}_{f} 0.34$ (Ethyl acetate/hexane, 1/ 3); Anal. calcd for $\mathrm{C}_{22} \mathrm{H}_{38} \mathrm{O}_{10} \mathrm{SSi}$ : C, 50.55; H, 7.33. Found: C, 50.73; H, 7.08.

\section{Analytic date for $S$-Phenyl-1-thio- $\beta$-glycosides}

Phenyl-3,4,6-tri- $O$-acetyl-2-deoxy-2-phthalimido-1-thio- $\beta$-D-glucopyranoside $3^{21}$

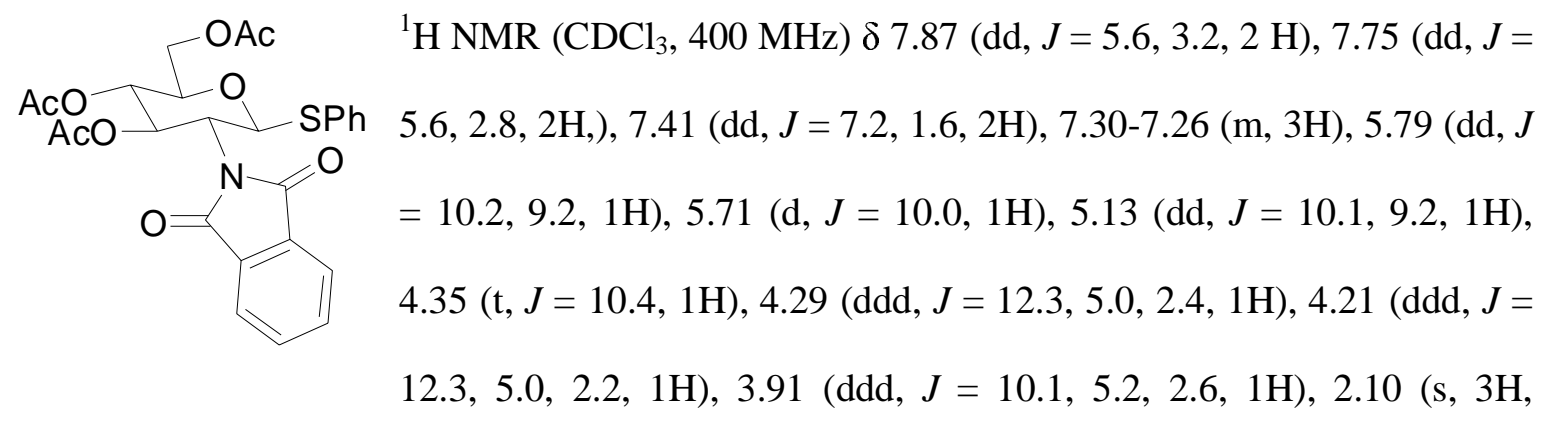

$\left.\mathrm{COCH}_{3}\right), 2.02\left(\mathrm{~s}, 3 \mathrm{H}, \mathrm{COCH}_{3}\right), 1.84\left(\mathrm{~s}, 3 \mathrm{H}, \mathrm{COCH}_{3}\right) ;{ }^{13} \mathrm{C} \mathrm{NMR}\left(\mathrm{CDCl}_{3}, 100 \mathrm{MHz}\right) \delta 170.6,170.1$, $169.4,167.8,166.9,134.4,134.3,133.3,131.6,131.2,131.0,128.9,128.4,123.7,83.1,75.9,71.6$, 68.8, 62.2, 53.6, 20.7, 20.6, 20.4; M.W. $\left(\mathrm{C}_{26} \mathrm{H}_{25} \mathrm{NO}_{9} \mathrm{~S}\right)$ 527.54; ESI-MS m/z 550 (17), $551\left(\mathrm{M}+\mathrm{Na}^{+}\right.$, 21); mp 142-143 ${ }^{\circ} \mathrm{C} ;[\alpha]_{\mathrm{D}}^{26}+56.6\left(c 1.0, \mathrm{CHCl}_{3}\right) ;$ TLC $\mathrm{R}_{f} 0.23$ (Ethyl acetate/hexane, 1/ 1).

\section{Phenyl 2, 3, 4, 6-tetra-O-acetyl-1-thio- $\beta$-D-galactopyranoside $4^{22}$}

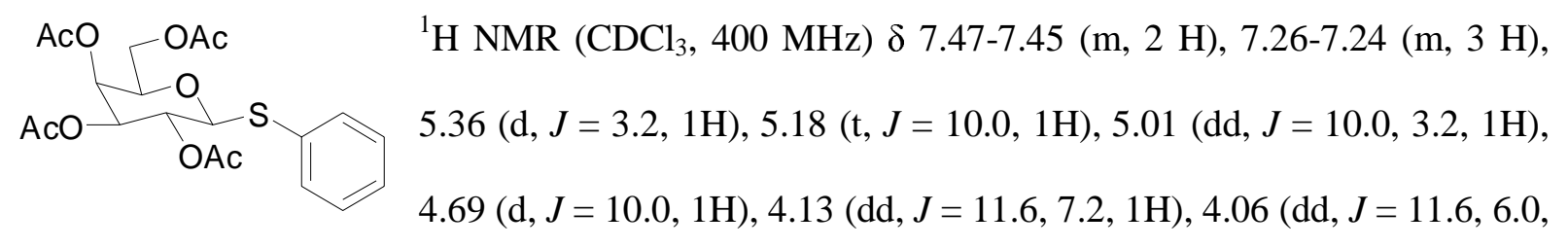

$1 \mathrm{H}), 3.91(\mathrm{t}, J=6.6,1 \mathrm{H}), 2.05\left(\mathrm{~s}, 3 \mathrm{H}, \mathrm{COCH}_{3}\right), 2.03\left(\mathrm{~s}, 3 \mathrm{H}, \mathrm{COCH}_{3}\right), 1.97\left(\mathrm{~s}, 3 \mathrm{H}, \mathrm{COCH}_{3}\right), 1.91(\mathrm{~s}$, 
$\left.3 \mathrm{H}, \mathrm{COCH}_{3}\right) ;{ }^{13} \mathrm{C} \mathrm{NMR}\left(\mathrm{CDCl}_{3}, 100 \mathrm{MHz}\right) \delta 170.0,169.8,169.6,169.1,132.2,128.6,127.8,86.1$, 74.1, 71.7, 67.0, 61.4, 20.5, 20.32, 20.27, 20.1; M.W. $\left(\mathrm{C}_{20} \mathrm{H}_{24} \mathrm{O}_{9} \mathrm{~S}\right)$ 440.46; ESI-MS m/z 463 $\left(\mathrm{M}+\mathrm{Na}^{+}, 100\right), 464(73) ; \mathrm{mp} 65-67^{\circ} \mathrm{C} ;[\alpha]_{\mathrm{D}}^{26}+4.2\left(c 1.0, \mathrm{CHCl}_{3}\right) ;$ TLC $\mathrm{R}_{f} 0.33$ (Ethyl acetate/hexane, 1/2).

Phenyl 2, 3, 4, 6-tetra- $O$-acetyl-1-thio- $\alpha$-D-mannopyranoside $5^{21}$

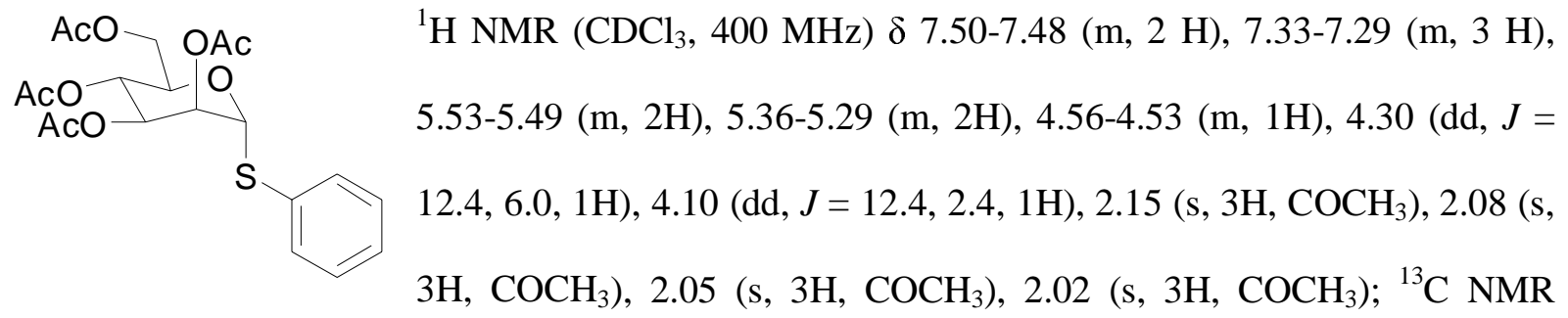
$\left(\mathrm{CDCl}_{3}, 100 \mathrm{MHz}\right) \delta 170.5,169.9,169.8,169.1,132.6,132.0,129.2,128.1,85.7,70.9,69.5,69.4$, 66.4, 62.4, 20.9, 20.69, 20.67, 20.6; M.W. $\left(\mathrm{C}_{20} \mathrm{H}_{24} \mathrm{O}_{9} \mathrm{~S}\right)$ 440.46; ESI-MS m/z $463\left(\mathrm{M}+\mathrm{Na}^{+}, 79\right), 464$ (57); $[\alpha]_{\mathrm{D}}^{24}+85.6\left(c 2.0, \mathrm{CH}_{2} \mathrm{Cl}_{2}\right) ;$ TLC $\mathrm{R}_{f} 0.27$ (Ethyl acetate/hexane, 1/ 3).

\section{Phenyl 2, 3, 5-tetra- $O$-acetyl- $\beta$-D-ribofuranoside $6^{23}$}

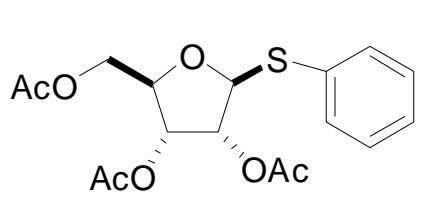

${ }^{1} \mathrm{H}$ NMR $\left(\mathrm{CDCl}_{3}, 400 \mathrm{MHz}\right) \delta$ 7.53-7.51 (m, $\left.2 \mathrm{H}\right)$, 7.34-7.32 (m, $\left.3 \mathrm{H}\right)$, $5.31(\mathrm{~d}, J=4.8,1 \mathrm{H}), 5.26-5.19(\mathrm{~m}, 2 \mathrm{H}), 4.27-4.25(\mathrm{~m}, 2 \mathrm{H}), 4.09(\mathrm{dd}$, $J=12.8,5.2,1 \mathrm{H}), 2.09\left(\mathrm{~s}, 3 \mathrm{H}, \mathrm{COCH}_{3}\right), 2.07\left(\mathrm{~s}, 3 \mathrm{H}, \mathrm{COCH}_{3}\right), 2.06(\mathrm{~s}$, $\left.3 \mathrm{H}, \mathrm{COCH}_{3}\right) ;{ }^{13} \mathrm{C} \mathrm{NMR}\left(\mathrm{CDCl}_{3}, 100 \mathrm{MHz}\right) \delta 170.5,169.6,169.4,133.4,131.7,129.0,128.4,87.9$, 80.1, 73.9, 71.4, 63.4, 20.8, 20.5; M.W. $\left(\mathrm{C}_{17} \mathrm{H}_{20} \mathrm{O}_{7} \mathrm{~S}\right)$ 368.40; ESI-MS m/z $391\left(\mathrm{M}+\mathrm{Na}^{+}, 53\right), 392$ (42); $[\alpha]_{\mathrm{D}}^{27}+12.3\left(c\right.$ 1.0, $\left.\mathrm{CHCl}_{3}\right)$; TLC $\mathrm{R}_{f} 0.31$ (Ethyl acetate/hexane, 1/ 3).

\section{phenyl 2, 3, 4-tri- $O$-acetyl-1-thio- $\beta$-D-xylopyranoside $7^{24}$}

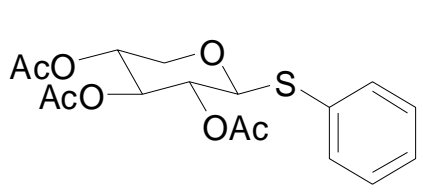

${ }^{1} \mathrm{H}$ NMR $\left(\mathrm{CDCl}_{3}, 400 \mathrm{MHz}\right) \delta$ 7.50-7.47 (m, $\left.2 \mathrm{H}\right)$, 7.33-7.31 (m, $\left.3 \mathrm{H}\right)$, $5.19(\mathrm{t}, J=8.2,1 \mathrm{H}), 4.90(\mathrm{t}, J=8.2,1 \mathrm{H}), 4.92-4.89(\mathrm{~m}, 1 \mathrm{H}) .4 .80(\mathrm{dd}$, 
$J=8.3,1 \mathrm{H}), 4.28(\mathrm{dd}, J=11.8,4.9,1 \mathrm{H}), 3.42(\mathrm{dd}, J=12.0,8.8,1 \mathrm{H}), 2.11\left(\mathrm{~s}, 3 \mathrm{H}, \mathrm{COCH}_{3}\right), 2.04(\mathrm{~s}$

$\left.6 \mathrm{H}, 2 \times \mathrm{COCH}_{3}\right) ;{ }^{13} \mathrm{C} \mathrm{NMR}\left(\mathrm{CDCl}_{3}, 100 \mathrm{MHz}\right) \delta 169.9,169.8,169.3,132.7,132.2,129.0,128.2$, 86.2, 72.0, 69.8, 68.4, 65.2, 20.8, 20.7; M.W. $\left(\mathrm{C}_{17} \mathrm{H}_{20} \mathrm{O}_{7} \mathrm{~S}\right)$ 368.40; ESI-MS m/z $391\left(\mathrm{M}+\mathrm{Na}^{+}, 50\right)$, 392 (41); $\mathrm{mp} 76-77{ }^{\circ} \mathrm{C} ;[\alpha]_{\mathrm{D}}^{26}-56.6\left(c\right.$ 1.0, $\left.\mathrm{CHCl}_{3}\right)$; TLC $\mathrm{R}_{f} 0.26$ (Ethyl acetate/hexane, 1/ 3).

\section{Phenyl 2, 3, 4-tri- $O$-acetyl-1-thio- $\beta$-L-arabinopyranoside $8^{25}$}

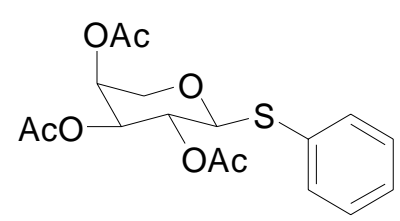

${ }^{1} \mathrm{H}$ NMR $\left(\mathrm{CDCl}_{3}, 400 \mathrm{MHz}\right) \delta$ 7.49-7.46 (m, $\left.2 \mathrm{H}\right)$ 7.28-7.27 (m, $\left.3 \mathrm{H}\right)$, 5.26-5.20 (m, 1H), $5.23(\mathrm{t}, J=8.2,1 \mathrm{H}), 5.09(\mathrm{dd}, J=8.4,3.4,1 \mathrm{H}), 4.80$ $(\mathrm{d}, J=7.8,1 \mathrm{H}), 4.12(\mathrm{dd}, J=12.7,4.2,1 \mathrm{H}), 3.65(\mathrm{dd}, J=12.7,2.0,1 \mathrm{H})$, $2.06\left(\mathrm{~s}, 6 \mathrm{H}, 2 \times \mathrm{COCH}_{3}\right), 2.02\left(\mathrm{~s}, 3 \mathrm{H}, \mathrm{COCH}_{3}\right) ;{ }^{13} \mathrm{C} \mathrm{NMR}\left(\mathrm{CDCl}_{3}, 100 \mathrm{MHz}\right) \delta 169.9,169.7,169.2$ $133.1,132.1,128.8,127.7,86.5,70.3,68.3,67.4,65.1,20.63,20.61,20.5 ;$ M.W. $\left(\mathrm{C}_{17} \mathrm{H}_{20} \mathrm{O}_{7} \mathrm{~S}\right)$ 368.40; ESI-MS m/z $391\left(\mathrm{M}+\mathrm{Na}^{+}, 50\right), 392(41) ;[\alpha]_{\mathrm{D}}^{26}-58.6\left(c\right.$ 1.0, $\left.\mathrm{CH}_{2} \mathrm{Cl}_{2}\right) ;$ TLC $\mathrm{R}_{f} 0.28$ (Ethyl acetate/hexane, 1/3).

\section{Phenyl 4, 6-di- $O$-acetyl-2, 3-dideoxy-1-thio-D-erythro-hex-2-enopyranoside $9^{26}$}

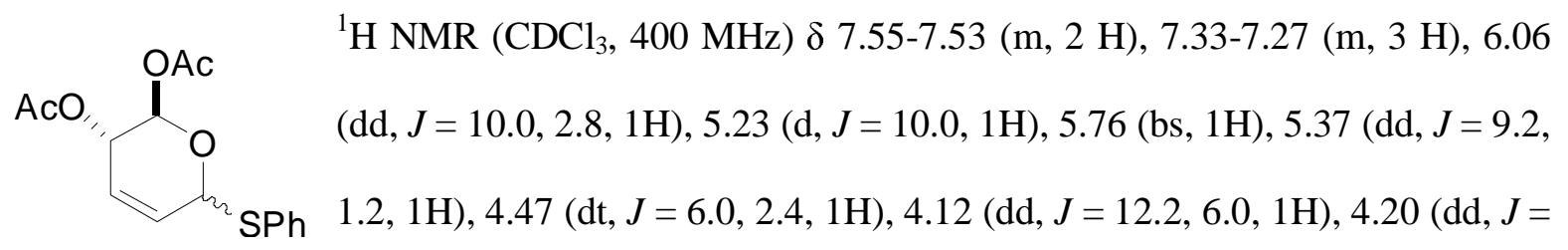

12.0, 2.0, 1H), 2.06 (major, s, 3H, $\mathrm{COCH}_{3}$ ), 2.07 (major, s, 3H, $\mathrm{COCH}_{3}$ ), 2.00 (minor, s, 3H, $\mathrm{COCH}_{3}$ ), 1.94 (minor, s, 3H, $\left.\mathrm{COCH}_{3}\right) ;{ }^{13} \mathrm{C} \mathrm{NMR}\left(\mathrm{CDCl}_{3}, 100 \mathrm{MHz}\right) \delta 170.7,170.2,134.7,131.7$, 128.9, 128.5, 127.8, 127.6, 83.0, 67.2, 65.1, 63.0, 20.9, 20.7; M.W. $\left(\mathrm{C}_{16} \mathrm{H}_{18} \mathrm{O}_{3} \mathrm{~S}\right)$ 322.37; ESI-MS $m / z 345\left(\mathrm{M}+\mathrm{Na}^{+}, 100\right), 346(45) ; \mathrm{mp} 64-65{ }^{\circ} \mathrm{C} ;[\alpha]_{\mathrm{D}}^{26}+24.4\left(c\right.$ 5.0, $\left.\mathrm{CHCl}_{3}\right) ;$ TLC $\mathrm{R}_{f} 0.34$ (Ethyl acetate/hexane, 1/3).

Phenyl 2, 3, 6-tri- $O$-acetyl-4- $O$-( 2', 3', 4', 6'-tetra-O-acetyl- $\alpha$-D-glucopyranosyl)-1-thio- $\beta$-Dglucopyranoside $10^{27}$

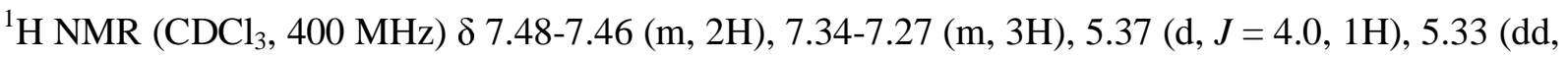




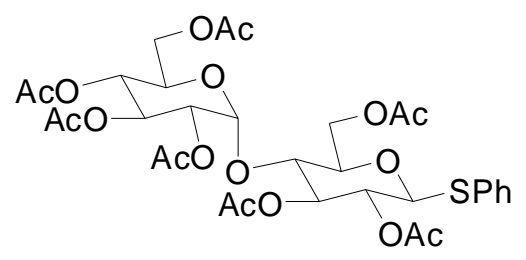
$J=10.3,9.7,1 \mathrm{H}), 5.27(\mathrm{t}, J=8.9,1 \mathrm{H}), 5.02(\mathrm{dd}, J=10.0,9.7$, $1 \mathrm{H}), 4.89-4.79(\mathrm{~m}, 2 \mathrm{H}), 4.52(\mathrm{~d}, J=10.0,1 \mathrm{H}), 4.27-4.21(\mathrm{~m}, 2 \mathrm{H})$, $4.16(\mathrm{dd}, J=12.4,7.7,1 \mathrm{H}), 4.03(\mathrm{dd}, J=12.4,2.1,1 \mathrm{H}), 3.95-3.87$ (m, 2H), 3.70 (ddd, $J=7.5,4.8,2.6,1 \mathrm{H}), 2.11\left(\mathrm{~s}, 3 \mathrm{H}, \mathrm{COCH}_{3}\right), 2.09$ (s, 3H, $\left.\mathrm{COCH}_{3}\right), 2.05(\mathrm{~s}, 3 \mathrm{H}$, $\left.\mathrm{COCH}_{3}\right), 2.02\left(\mathrm{~s}, 3 \mathrm{H}, \mathrm{COCH}_{3}\right), 2.01\left(\mathrm{~s}, 3 \mathrm{H}, \mathrm{COCH}_{3}\right), 1.98\left(\mathrm{~s}, 3 \mathrm{H}, \mathrm{COCH}_{3}\right), 1.97\left(\mathrm{~s}, 3 \mathrm{H}, \mathrm{COCH}_{3}\right)$; ${ }^{13} \mathrm{C} \mathrm{NMR}\left(\mathrm{CDCl}_{3}, 100 \mathrm{MHz}\right) \delta 170.49,170.47,170.3,170.1,169.9,169.5,169.4,133.4,131.3$, $128.9,128.4,95.6,85.1,76.5,76.1,72.6,70.7,70.0,69.3,68.6,68.1,62.9,61.6,20.9,20.8,20.7$, 20.6, 20.5; M.W. $\left(\mathrm{C}_{32} \mathrm{H}_{40} \mathrm{O}_{17} \mathrm{~S}\right)$ 728.72; ESI-MS $m / z 751\left(\mathrm{M}+\mathrm{Na}^{+}, 13\right) ; \mathrm{mp} 89-90{ }^{\circ} \mathrm{C} ;[\alpha]_{\mathrm{D}}^{25}+44.2(c$ 1.0, $\mathrm{CHCl}_{3}$ ); TLC $\mathrm{R}_{f} 0.12$ (Ethyl acetate/hexane, 1/ 1).

\section{Phenyl 2, 3, 6-tri- $O$-acetyl-4- $O$-( 2', 3', 4', 6'-tetra- $O$-acetyl- $\beta$-D-galactopyrano -syl )-1-thio-}

\section{$\beta$-D-glucopyranoside $11^{28}$}

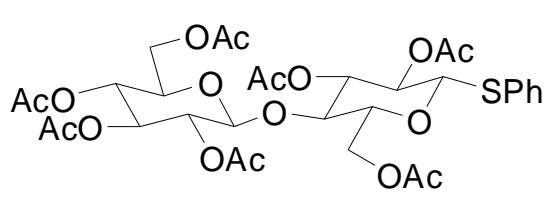

${ }^{1} \mathrm{H}$ NMR $\left(\mathrm{CDCl}_{3}, 400 \mathrm{MHz}\right) \delta$ 7.49-7.46 (m, 2H), 7.33-7.27

$(\mathrm{m}, 3 \mathrm{H}), 5.34(\mathrm{dd}, J=3.4,1.0,1 \mathrm{H}), 5.23(\mathrm{t}, J=9.2,1 \mathrm{H}), 5.09$ $(\mathrm{dd}, J=10.4,7.8,1 \mathrm{H}), 4.94(\mathrm{dd}, J=10.4,3.4,1 \mathrm{H}), 4.88(\mathrm{dd}$,

$J=10.0,9.2,1 \mathrm{H}), 4.66(\mathrm{~d}, J=10.1,1 \mathrm{H}), 4.52(\mathrm{dd}, J=12.0,2.0,1 \mathrm{H}), 4.46(\mathrm{~d}, J=8.0,1 \mathrm{H})$ 4.14-4.04 (m, 3H), 3.85 (bt, $J=7.2,1 \mathrm{H}), 3.63-3.62(\mathrm{~m}, 2 \mathrm{H}), 2.15\left(\mathrm{~s}, 3 \mathrm{H}, \mathrm{COCH}_{3}\right), 2.10(\mathrm{~s}, 3 \mathrm{H}$, $\left.\mathrm{COCH}_{3}\right), 2.09\left(\mathrm{~s}, 3 \mathrm{H}, \mathrm{COCH}_{3}\right), 2.04\left(\mathrm{~s}, 6 \mathrm{H}, 2 \times \mathrm{COCH}_{3}\right), 2.03\left(\mathrm{~s}, 3 \mathrm{H}, \mathrm{COCH}_{3}\right), 1.95(\mathrm{~s}, 3 \mathrm{H}$, $\left.\mathrm{COCH}_{3}\right) ;{ }^{13} \mathrm{C} \mathrm{NMR}\left(\mathrm{CDCl}_{3}, 100 \mathrm{MHz}\right) \delta 170.21,170.16,170.0,169.9,169.6,169.5,169.0,133.0$, $131.7,128.8,128.2,100.9,85.4,76.1,73.8,70.9,70.7,70.3,69.1,66.6,62.1,60.8,20.72,20.67$, 20.5, 20.4; M.W. $\left(\mathrm{C}_{32} \mathrm{H}_{40} \mathrm{O}_{17} \mathrm{~S}\right)$ 728.72; ESI-MS m/z $752\left(\mathrm{M}+\mathrm{Na}^{+}, 48\right), 753$ (43); mp 170-171 ${ }^{\circ} \mathrm{C}$; $[\alpha]_{\mathrm{D}}^{25}-19.6\left(c 1.0, \mathrm{CHCl}_{3}\right) ;$ TLC $\mathrm{R}_{f} 0.13$ (Ethyl acetate/hexane, 1/ 1). 
p-Tolyl-1-thio- $\beta$-D-glucopyranoside-12,29

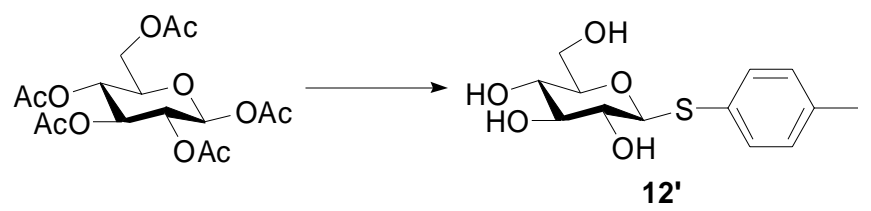

In a flame-dried, 25-mL, two-necked, round-bottomed flask equipped with an addition funnel, under argon atmosphere, was placed $\mathrm{MoO}_{2} \mathrm{Cl}_{2}(11.8 \mathrm{mg}, 0.06 \mathrm{mmol}, 3 \mathrm{~mol} \%)$ in anhydrous $\mathrm{CH}_{2} \mathrm{Cl}_{2}$ ( $3 \mathrm{~mL}$ ). To the catalyst solution, a solution of petaacetyl- $\beta$-D-glucose ( $880 \mathrm{mg}, 2 \mathrm{mmol})$ in $\mathrm{CH}_{2} \mathrm{Cl}_{2}$ $(5 \mathrm{~mL})$ was slowly added at ambient temperature via the addition funnel. After $10 \mathrm{~min}$, a solution of 4-methyl-thiophenol (323 mg, $2.6 \mathrm{mmol}, 2.6$ equiv) in $\mathrm{CH}_{2} \mathrm{Cl}_{2}(2 \mathrm{~mL})$ was slowly added to the reaction mixture. After completion of the reaction (about $14 \mathrm{~h}$ ), the reaction mixture was charged with a solution of $\mathrm{MoO}_{2} \mathrm{Cl}_{2}(1.9 \mathrm{mg}, 0.02 \mathrm{mmol}, 1 \mathrm{~mol} \%)$ in anhydrous ethanol $(5 \mathrm{~mL})$ and then heated to reflux for $12 \mathrm{~h}$. After having been cooled to ambient temperature, the mixture was evaporated to dryness under reduced pressure, and the residue was solidified by dilution with 20 $\mathrm{mL}$ of warm diethyl ether. The resulting solid was filtered and washed with diethyl ether $(2 \times 20$ $\mathrm{mL})$. The resultant white solid was dissolved in ethyl alcohol $(20 \mathrm{~mL})$ and the solution was filtered through a short plug of silica gel. The filtrate was concentrated to give $556 \mathrm{mg}(94 \%)$ of 12' as white crystals: ${ }^{1} \mathrm{H}$ NMR $\left(\mathrm{CDCl}_{3}, 400 \mathrm{MHz}\right) \delta 7.46(\mathrm{~d}, J=8.0,2 \mathrm{H}), 7.13(\mathrm{~d}, J=8.0,2 \mathrm{H}), 4.58$ (bs, $2 \mathrm{H}, \mathrm{OH}), 4.52(\mathrm{~d}, J=7.4,1 \mathrm{H}), 3.86(\mathrm{dd}, J=11.0,1.4,1 \mathrm{H}), 3.67(\mathrm{dd}, J=11.1,4.9,1 \mathrm{H}), 3.41(\mathrm{t}, J=$ 1.9, 1H), 3.36-3.26 (m, 2H), $3.20(\mathrm{t}, J=9.6,1 \mathrm{H}),{ }^{13} \mathrm{C}$ NMR (DMSO-d $\left.6,100 \mathrm{MHz}\right) \delta 138.9,133.6$, 131.2, 130.7, 89.7, 82.1, 79.7, 73.8, 71.5, 63.0, 21.2; M.W. $\left(\mathrm{C}_{13} \mathrm{H}_{18} \mathrm{O}_{5} \mathrm{~S}\right)$ 286.34; ESI-MS m/z 309 $\left(\mathrm{M}+\mathrm{Na}^{+}, 48\right), 310(20) ; \mathrm{mp} 147-148{ }^{\circ} \mathrm{C} ;[\alpha]_{\mathrm{D}}^{22}+32.0$ (c 1.0, acetone); TLC $\mathrm{R}_{f} 0.14$ (EtOAc/hexane, 2/ 1). 
4-Methylphenyl 4, 6-O-benzylidine-1-thio- $\beta$-D-glucopyranoside-12 $2^{30}$

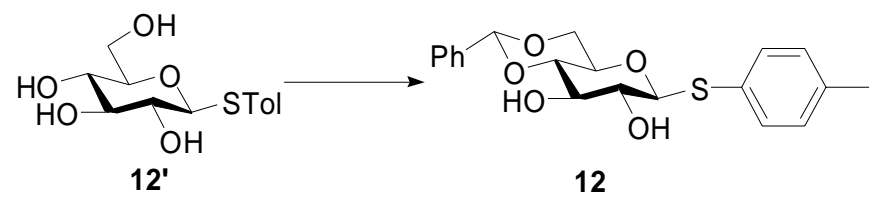

In a dry 50-mL, two-necked, round-bottomed flask was placed $\mathrm{MoO}_{2} \mathrm{Cl}_{2}(17.3 \mathrm{mg}, 0.087$ $\mathrm{mmol}, 5 \mathrm{~mol} \%)$ in anhydrous 1,4-dioxane $(5 \mathrm{~mL})$. To the above solution, benezaldehyde $(267.5 \mathrm{mg}$, $273 \mu \mathrm{L}, 2.62 \mathrm{mmol}, 1.5$ equiv) was slowly added via a micro-syringe at ambient temperature. After $20 \mathrm{~min}, p$-tolyl-1-thio- $\beta$-D-glucopyranoside $(500 \mathrm{mg}, 1.75 \mathrm{mmol})$ was added to the above solution in one portion. The resulting reaction mixture was stirred the reaction for 20-24 hours. After completion of the reaction as evidenced by TLC analysis, the reaction mixture was sequentially treated with $\mathrm{Et}_{3} \mathrm{~N}(0.2 \mathrm{~mL})$ and cold saturated aqueous $\mathrm{NaHCO}_{3}(5 \mathrm{~mL})$. The organic layer was separated and the aqueous layer was further extracted ethyl acetate $(50 \mathrm{~mL})$. The combined organic layer was washed with brine, dried $\left(\mathrm{MgSO}_{4}\right)$, filtered, and evaporated. The resultant crude product was purified by column chromatography (ethyl acetate/hexane, 1/2; with $1 \% \mathrm{Et}_{3} \mathrm{~N}$ ) to give $524 \mathrm{mg}$ of acetal-12 (80\%) as a white solid: ${ }^{1} \mathrm{H}$ NMR $\left(400 \mathrm{MHz}, \mathrm{CDCl}_{3}\right) \delta$ 7.48-7.43 (m, 4H), 7.38-7.35 (m, $3 \mathrm{H}), 7.15(\mathrm{~d}, J=8.0,2 \mathrm{H}), 5.53(\mathrm{~s}, 1 \mathrm{H}), 4.58(\mathrm{~d}, J=9.6,1 \mathrm{H}), 4.37(\mathrm{dd}, J=10.7,4.4,1 \mathrm{H}), 3.85(\mathrm{dd}$, $J=7.5,2.2,1 \mathrm{H}), 3.77(\mathrm{dd}, J=10.7,4.4,1 \mathrm{H}), 3.52-3.48(\mathrm{~m}, 2 \mathrm{H}), 3.43(\mathrm{dd}, J=4.4,2.2,1 \mathrm{H}), 2.70$ (br, 1H, OH), $2.60(\mathrm{br}, 1 \mathrm{H}, \mathrm{OH}), 2.36\left(\mathrm{~s}, 3 \mathrm{H}, \mathrm{CH}_{3}\right) ;{ }^{13} \mathrm{C} \mathrm{NMR}\left(100 \mathrm{MHz}, \mathrm{CDCl}_{3}\right) \delta 138.9,136.9$, $133.7,129.9,129.3,128.3,127.2,126.3,101.9,88.7,80.3,76.7,74.6,72.5,70.6,68.6,21.2$; M.W. $\left(\mathrm{C}_{20} \mathrm{H}_{22} \mathrm{O}_{5} \mathrm{~S}\right)$ 374; MS (EI, $\left.70 \mathrm{eV}\right) 374$ (M+34), 250 (100), 145 (10), 127 (19), 124 (77), 107 (71), 91 (43), 79 (25); $[\alpha]_{\mathrm{D}}^{22}-34.40\left(c 1.0, \mathrm{CHCl}_{3}\right)$; TLC $\mathrm{R}_{f} 0.21$ (Ethyl acetate/hexane, 1/ 2).

\section{Preparation of thioglycosidic disaccharide-15}

\section{Methyl 6-S-Acetyl-2,3,4-tri- $O$-benzyl-6-thio- $\alpha$-D-glucopyranoside ${ }^{31}$}

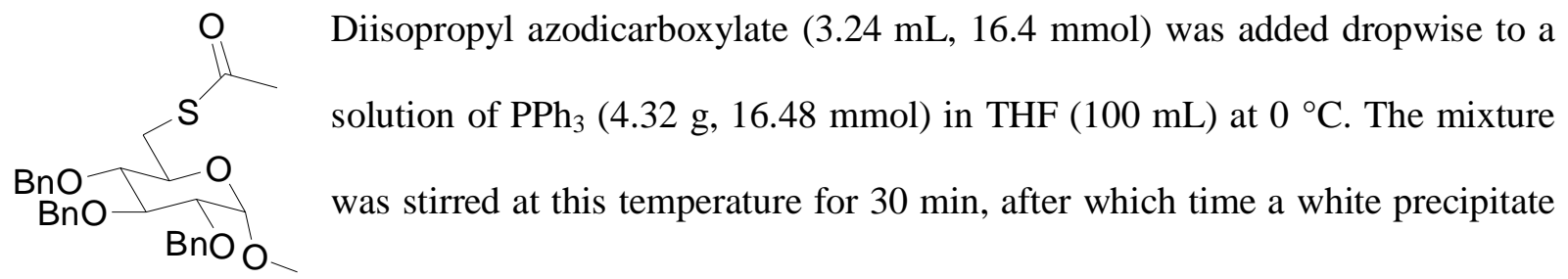


was formed. A solution of methyl-2,3,4-tri- $O$-benzyl- $\alpha$-D-glucopyranoside ${ }^{32}(3.808 \mathrm{~g}, 8.2 \mathrm{mmol})$ and thioacetic acid (1.2 mL, $16.4 \mathrm{mmol}, 2$ equiv) in THF (100 mL) was then added. The reaction mixture was stirred at $0{ }^{\circ} \mathrm{C}$ for $1 \mathrm{~h}$ and then allowed to warm to ambient temperature overnight. The reaction mixture was evaporated and the resulting red residue was purified by column chromatography. The product was further purified by re-crystallization from hexane to afford methyl 6-S-acetyl-2,3,4-tri- $O$-benzyl-6-thio- $\alpha$-D-glucopyranoside as a crystalline solid $(2.657 \mathrm{~g}$, 62\%): ${ }^{1} \mathrm{H}$ NMR $\left(\mathrm{CDCl}_{3}, 400 \mathrm{MHz}\right) \delta$ 7.35-7.24 (m, $\left.15 \mathrm{H}\right), 4.98\left(\mathrm{~d}, J=10.8,1 \mathrm{H}, \mathrm{OCH}_{a} \mathrm{H}_{\mathrm{b}} \mathrm{Ph}\right), 4.89$ $\left(\mathrm{d}, J=10.8, \mathrm{OCH}_{\mathrm{a}} H_{b} \mathrm{Ph}\right), 4.81\left(\mathrm{~d}, J=11.0,1 \mathrm{H}, \mathrm{OCH}_{a} \mathrm{H}_{\mathrm{b}} \mathrm{Ph}\right), 4.78\left(\mathrm{~d}, J=11.0,1 \mathrm{H}, \mathrm{OCH}_{\mathrm{a}} H_{b} \mathrm{Ph}\right)$, $4.65\left(\mathrm{~d}, J=12.0,1 \mathrm{H}, \mathrm{OCH}_{a} \mathrm{H}_{\mathrm{b}} \mathrm{Ph}\right), 4.62\left(\mathrm{~d}, J=12.0,1 \mathrm{H}, \mathrm{OCH}_{\mathrm{a}} H_{b} \mathrm{Ph}\right), 4.54(\mathrm{~d}, J=3.2,1 \mathrm{H}, \mathrm{HC}(1))$, $3.97(\mathrm{~d}, J=9.2,1 \mathrm{H}), 3.75(\mathrm{td}, J=7.5,2.2,1 \mathrm{H}), 3.51(\mathrm{dd}, J=9.6,3.3,1 \mathrm{H}), 3.43(\mathrm{dd}, J=13.6,2.3$, $1 \mathrm{H}), 3.36\left(\mathrm{~s}, 3 \mathrm{H}, \mathrm{OCH}_{3}\right), 3.30(\mathrm{t}, J=9.3,1 \mathrm{H}), 3.03(\mathrm{dd}, J=13.7,7.8,1 \mathrm{H}), 2.34\left(\mathrm{~s}, 3 \mathrm{H}, \mathrm{CH}_{3}\right) ;{ }^{13} \mathrm{C}$ $\operatorname{NMR}\left(\mathrm{CDCl}_{3}, 100 \mathrm{MHz}\right) \delta 194.8,138.6,138.0,137.9,128.41,128.36,128.1,128.0,127.92,127.89$, 127.8, 127.6, 97.8, 81.8, 80.5, 79.9, 75.7, 75.1, 73.3, 69.3, 55.1, 30.8, 30.5; M.W. $\left(\mathrm{C}_{30} \mathrm{H}_{34} \mathrm{O}_{6} \mathrm{~S}\right)$ 522.65; ESI-MS m/z $545\left(\mathrm{M}+\mathrm{Na}^{+}, 29\right) ; \mathrm{mp} \mathrm{82-83}{ }^{\circ} \mathrm{C} ;[\alpha]_{\mathrm{D}}^{22}+24.4\left(c \quad 1.0, \mathrm{CHCl}_{3}\right) ;$ TLC $\mathrm{R}_{f} 0.51$ (Ethyl acetate/hexane, 1/ 3).

\section{Methyl 2,3,4-tri-O-benzyl-6-thio- $\alpha$-D-glucopyranoside-14 ${ }^{31 a}$}

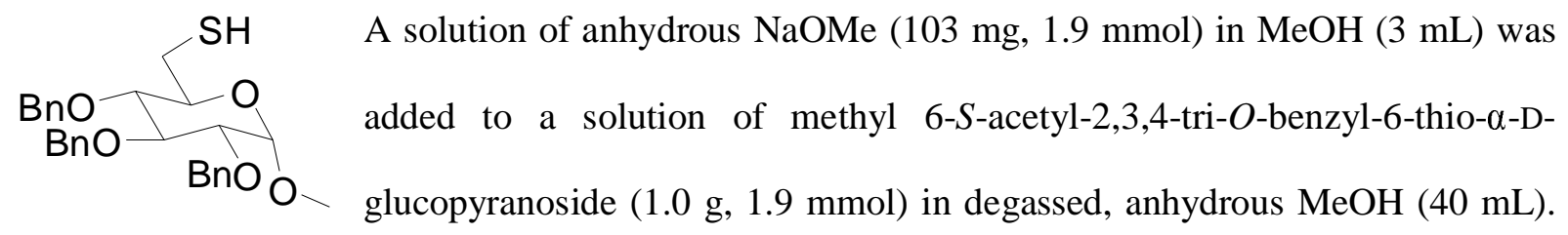

The reaction mixture was stirred for $2 \mathrm{~h}$. Amberlyst-15 ion-exchange resin was then added until the $\mathrm{pH}$ of the solution became neutral. The resin in the reaction mixture was removed by filtration and the collected filtrate was evaporated. The resulting residue was purified by column chromatography under nitrogen atmosphere to afford $483 \mathrm{mg}(53 \%)$ of $\mathbf{1 4}$ as a colorless oil: ${ }^{1} \mathrm{H} \mathrm{NMR}\left(\mathrm{CDCl}_{3}, 400\right.$ $\mathrm{MHz}) \delta 7.34-7.29(\mathrm{~m}, 15 \mathrm{H}), 4.99\left(\mathrm{~d}, J=10.8,1 \mathrm{H}, \mathrm{OCH}_{\mathrm{a}} \mathrm{H}_{\mathrm{b}} \mathrm{Ph}\right), 4.89(\mathrm{~d}, J=10.8,1 \mathrm{H}$, $\left.\mathrm{OCH}_{\mathrm{a}} H_{b} \mathrm{Ph}\right), 4.80\left(\mathrm{~d}, J=12.6,1 \mathrm{H}, \mathrm{OCH}_{\mathrm{a}} \mathrm{H}_{\mathrm{b}} \mathrm{Ph}\right), 4.77\left(\mathrm{~d}, J=12.6,1 \mathrm{H}, \mathrm{OCH}_{\mathrm{a}} H_{b} \mathrm{Ph}\right), 4.63(\mathrm{~d}, J=$ 
13.6, $\left.1 \mathrm{H}, \mathrm{OCH}_{\mathrm{a}} \mathrm{H}_{\mathrm{b}} \mathrm{Ph}\right), 4.63\left(\mathrm{dd}, J=13.6,1 \mathrm{H}, \mathrm{OCH}_{\mathrm{a}} H_{b} \mathrm{Ph}\right), 4.56(\mathrm{~d}, J=3.6, \mathrm{HC}(1) 1 \mathrm{H}), 3.98(\mathrm{~d}, J$ $=8.8,1 \mathrm{H}), 3.87(\mathrm{t}, J=8.4,1 \mathrm{H}), 3.51(\mathrm{dd}, J=9.2,3.2,1 \mathrm{H}), 3.38\left(\mathrm{~s}, 3 \mathrm{H}, \mathrm{OCH}_{3}\right), 3.31(\mathrm{t}, J=9.2,1 \mathrm{H})$, $3.16(\mathrm{dd}, J=13.6,1.6,1 \mathrm{H}), 2.76(\mathrm{dd}, J=13.2,8.7,1 \mathrm{H}), 1.28(\mathrm{t}, J=8.8,1 \mathrm{H}, \mathrm{SH}) ;{ }^{13} \mathrm{C} \mathrm{NMR}\left(\mathrm{CDCl}_{3}\right.$, $100 \mathrm{MHz}) \delta 138.6,138.0,128.43,128.40,128.37,128.1,127.95,127.90,127.8,127.6,97.8,81.9$,

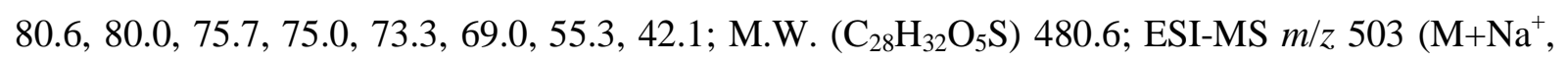
53); $[\alpha]_{\mathrm{D}}^{22}+58.6\left(c 0.9, \mathrm{CHCl}_{3}\right) ;$ TLC $\mathrm{R}_{f} 0.41$ (Ethyl acetate/hexane, 1/ 3).

\section{Methyl 2,3,4- tri-O-benzyl -6-S-(2',3',4',6'-tetra-O-acetyl - $\beta$-D- glucopyranosyl-6-thio- $\alpha$-D-} glucopyranoside-15

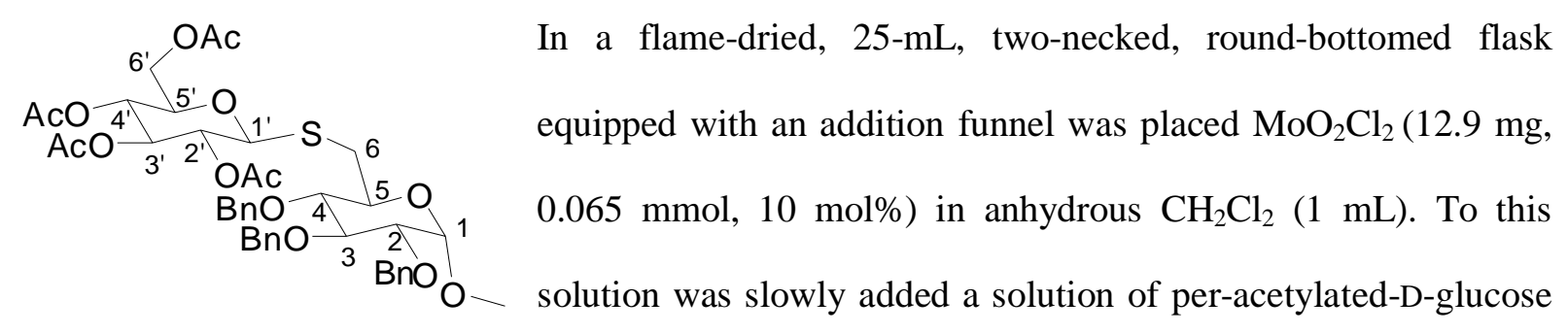

-2a (329 mg, $0.837 \mathrm{mmol}, 1.3$ equiv) in $\mathrm{CH}_{2} \mathrm{Cl}_{2}(2 \mathrm{~mL}$ ) at ambient temperature via the addition funnel. After $10 \mathrm{~min}$, the reaction was slowly treated with a solution of methyl 2,3,4-tri-O-benzyl-6thio- $\alpha$-D-glucopyranoside-15 $(312 \mathrm{mg}, 0.649 \mathrm{mmol})$ in $\mathrm{CH}_{2} \mathrm{Cl}_{2}(2 \mathrm{~mL})$. After completion of the reaction as evidenced by TLC analysis $(18 \mathrm{~h})$, the reaction mixture was treated with cold saturated aqueous $\mathrm{NaHCO}_{3}(5 \mathrm{~mL})$. The organic layer was separated and washed with brine, dried $\left(\mathrm{MgSO}_{4}\right)$, filtered, and evaporated. The resultant crude residue was purified by column chromatography to afford $389 \mathrm{mg}$ of thioglycosidic disaccharide-15 in $74 \%$ yield. ${ }^{1} \mathrm{H} \mathrm{NMR}\left(\mathrm{CDCl}_{3}, 400 \mathrm{MHz}\right) \delta$ 7.37-7.27 (m, $15 \mathrm{H}), 5.19$ (t, $\left.J=9.6,1 \mathrm{H}, \mathrm{HC}\left(4^{\prime}\right)\right), 5.05$ (t, $\left.J=9.6,1 \mathrm{H}, \mathrm{HC}\left(3^{\prime}\right)\right), 4.95$ (t, $J=7.2,1 \mathrm{H}$, $\left.\mathrm{HC}\left(2^{\prime}\right)\right), 4.98\left(\mathrm{~d}, J=11.0,1 \mathrm{H}, \mathrm{OCH}_{a} \mathrm{H}_{\mathrm{b}} \mathrm{Ph}\right), 4.92\left(\mathrm{~d}, 1 \mathrm{H}, J=11.0, \mathrm{OCH}_{\mathrm{a}} H_{b} \mathrm{Ph}\right), 4.81-4.77(\mathrm{~m}, 2 \mathrm{H}$, $\left.\mathrm{OCH}_{2} \mathrm{Ph}\right), 4.66\left(\mathrm{~d}, J=13.2,1 \mathrm{H}, \mathrm{OCH}_{a} \mathrm{H}_{\mathrm{b}} \mathrm{Ph}\right), 4.64\left(\mathrm{~d}, J=13.2,1 \mathrm{H}, \mathrm{OCH}_{\mathrm{a}} H_{b} \mathrm{Ph}\right), 4.60(\mathrm{~d}, J=7.2$, $\left.1 \mathrm{H}, \mathrm{HC}\left(1^{\prime}\right)\right), 4.57$ (d, $\left.J=3.6,1 \mathrm{H}, \mathrm{HC}(1)\right), 4.18\left(\mathrm{dd}, J=12.4,4.8,1 \mathrm{H}, H_{a} \mathrm{H}_{\mathrm{b}} \mathrm{C}\left(6^{\prime}\right)\right), 4.05$ (dd, $J=$ 12.4, 2.0, $\left.1 \mathrm{H}, \mathrm{H}_{\mathrm{a}} H_{b} \mathrm{C}\left(6^{\prime}\right)\right), 3.96(\mathrm{t}, J=9.2,1 \mathrm{H}, \mathrm{HC}(3)), 3.83(\mathrm{td}, J=8.0,2.0,1 \mathrm{H}, \mathrm{HC}(5)), 3.59$ $\left(\mathrm{ddd}, J=10.0,4.4,2.0,1 \mathrm{H}, \mathrm{HC}\left(5^{\prime}\right)\right), 3.49(\mathrm{dd}, J=9.6,3.2,1 \mathrm{H}, \quad \mathrm{HC}(2)), 3.40\left(\mathrm{~s}, 3 \mathrm{H}, \mathrm{OCH}_{3}\right), 3.31$ 
$(\mathrm{t}, J=9.2,1 \mathrm{H}, \mathrm{HC}(4)), 3.00\left(\mathrm{dd}, J=13.6,2.0,1 \mathrm{H}, H_{a} \mathrm{H}_{\mathrm{b}} \mathrm{C}(6)\right), 2.69(\mathrm{dd}, J=13.6,8.8,1 \mathrm{H}$,

$\mathrm{H}_{\mathrm{a}} \mathrm{H}_{b} \mathrm{C}(6)$ ), 2.04 ((brs, 3H, $\mathrm{COCH}_{3}$ ), 2.02 (brs, 3H, $\mathrm{COCH}_{3}$ ), 2.01 (brs, 3H, $\mathrm{COCH}_{3}$ ), 2.00 (brs, 3H,

$\left.\mathrm{COCH}_{3}\right) ;{ }^{13} \mathrm{C} \mathrm{NMR}\left(\mathrm{CDCl}_{3}, 100 \mathrm{MHz}\right) \delta 170.6(\mathrm{C}=\mathrm{O}), 170.2(\mathrm{C}=\mathrm{O}), 169.3(\mathrm{C}=\mathrm{O}), 169.1(\mathrm{C}=\mathrm{O})$,

138.6, 138.2, 138.1, 128.47, 128.45, 128.37, 128.0, 127.94, 127.86, 127.83, 127.6, 97.8 (C(1)), 83.9

$\left(\mathrm{C}\left(1^{\prime}\right)\right), 81.9(\mathrm{C}(3)), 80.7(\mathrm{C}(4)), 79.9(\mathrm{C}(2)), 75.71\left(\mathrm{OCH}_{2} \mathrm{Ph}\right), 75.70\left(\mathrm{C}\left(5^{\prime}\right)\right), 75.0\left(\mathrm{OCH}_{2} \mathrm{Ph}\right), 73.9$

$(\mathrm{C}(4)), 73.3\left(\mathrm{OCH}_{2} \mathrm{Ph}\right), 71.0(\mathrm{C}(5)), 70.2\left(\mathrm{C}\left(2^{\prime}\right)\right), 68.3\left(\mathrm{C}\left(3^{\prime}\right), 62.0\left(\mathrm{C}\left(6^{\prime}\right), 55.1\left(\mathrm{OCH}_{3}\right), 31.6(\mathrm{C}(6))\right.\right.$,

$20.61\left(\mathrm{COCH}_{3}\right), 20.58\left(\mathrm{COCH}_{3}\right), 20.55\left(\mathrm{COCH}_{3}\right), 20.51\left(\mathrm{COCH}_{3}\right)$; M.W. $\left(\mathrm{C}_{42} \mathrm{H}_{50} \mathrm{O}_{14} \mathrm{~S}\right) 810.9$;

ESI-MS $m / z 833\left(\mathrm{M}+\mathrm{Na}^{+}, 100\right) ;[\alpha]_{\mathrm{D}}^{22}+14.6\left(c\right.$ 1.0, $\left.\mathrm{CHCl}_{3}\right) ;$ TLC $\mathrm{R}_{f} 0.38$ (Ethyl acetate/hexane, $1 /$

2); Anal. calcd for $\mathrm{C}_{42} \mathrm{H}_{50} \mathrm{O}_{14} \mathrm{~S}$ : C, 62.21; H, 6.21; S, 3.95. Found: C, 62.47; H, 5.98; S, 3.62.

\section{References:}

(1) Bretting, H.; Jacobs, G.; Benecke, I.; Koenig, W. A.; Thiem, J. Carbohydr. Res. 1985, 139, 225.

(2) (a) Hodosi, G.; Kovac, P. Carbohydr. Res. 1997, 303, 239. (b) Utille, J.-P.; Gagnaire, D. Carbohydr. Res. 1982, 106, 43.

(3) Ishii, H.; Kitagawa, I.; Matsushita, K.; Shirakawa, K.; Tori, K. Tetrahedron Lett. 1981, 22, 1529.

(4) Xue, J.; Pan, Y.; Guo, Z. Tetrahedron Lett. 2002, 43, 1599.

(5) Lee, E. E.; Wood, J. O. Carbohydr. Res. 1981, 89, 329-333.

(6) Yoshida, A.; Hayashi, T.; Takeda, N.; Oida, S.; Ohki, E. Chem. Pharm. Bull. 1981, 29, 1854.

(7) Magnusson, G.; Ahlfors, S.; Dahmen, J.; Jansson, K.; Nilsson, U. J. Org. Chem. 1990, 55, 3932.

(8) Cheng, M. S.; Wang, Q. L.; Tian, Q.; Song, H. Y.; Liu, Y. X.; Li, Q.; Xu, X.; Miao, H. D.; Yao, X. S.; Yang, Z. J. Org. Chem., 2003, 68, 3658.

(9) Kondo, H.; Aoki, S.; Ichikawa, Y.; Halcomb, R. L.; Ritzen, H.; Wong, C.-H. J. Org. Chem. 1994, 59, 864.

(10) Ding, Y.; Liu, Y. Carbohydr. Res. 1991, 209, 306.

(11) Pakulski, Z.; Pierozynski, D.; Zamojski, A. Tetrahedron, 1996, 50, 2975.

(12) Shimadate, T.; Chiba, S.; Inouye, K.; Iino, T.; Hosoyama, Y. Bull. Chem. Soc. Jpn. 1982, 55, 3552 .

(13) Caddick, S.; Gazzard, L.; Motherwell, W. B.; Wilkinson, J. A. Tetrahedron 1996, 52, 149.

(14) Griffin, G. W.; Bandara, N. C.; Clarke, M. A.; Tsang, W.-S.; Garegg, P. J. Heterocycles 1990, $30,939$.

(15) Ogawa; M. Carbohydr. Res. 1977, 54, C17-C19.

(16) Vic, G.; Hastings, J. J.; Howarth, O. W.; Crout, D. H. G. Tetrahedron: Asymmetry, 1996, 7, 709 . 
(17) Yanase, M.; Funabashi, M. J. Carbohydr. Chem. 2000, 19, 53.

(18) Chervin, S. M.; Abada, P.; Koreeda, M. Org. Lett. 2000, 2, 369.

(19) Suzuki, K.; Susaki, H.; Okuno, S.; Sugiyama, Y. J. Pharmacol. Exp. Ther. 1999, 288, 57.

(20) Xia, J.; Alderfer, J. L.; Piskorz, C. F.; Locke, R. D.; Matta, K. L. Carbohydr. Res. 2000, 328, 147.

(21) Fuente, J. M. de la; Penades, S. Tetrahedron: Asymmetry 2002, 13, 1879.

(22) Kametani, T.; Kawamura, K.; Honda, T. J. Am. Chem. Soc., 1987, 109, 3010.

(23) (a) Kim, J. M.; Roy, R. Carbohydr. Res. 1997, 298, 173. (b) Ref-22.

(24) Kim, J. M.; Roy, R. Carbohydr. Res. 1997, 298, 173.

(25) Yu, W.; Jin, Z. J. Am. Chem. Soc. 2001, 123, 3369.

(26) Raadt, A. de; Ferrier, R. J., Carbohydr. Res. 1991, 216, 93.

(27) Tropper, F. D.; Andersson, F. O.; Grand-Maitre, C.; Roy, R. Synthesis 1991, 9, 734.

(28) Zhang, Z.; Magnusson, G. Carbohydr. Res. 1996, 295, 41.

(29) Lin, C.-C.; Hsu, T.-S.; Lu, K.-C.; Huang, I-T. J. Chin. Chem. Soc. 2000, 47, 921.

(30) Wong, C.-H.; Ye, X.-S.; Zhang, Z. J. Am. Chem. Soc. 1998, 120, 7137.

(31) (a) Paterson, D. E.; Griffin, F. K.; Alcaraz, M.-L.; Taylor, R. J. K. Eur. J. Org. Chem. 2002, 7, 1323. (b) Kvaernoe, L.; Werder, M.; Hauser, H.; Carreira, E. M. Org. Lett. 2005, 6, 1145. 
H spectrum of

言

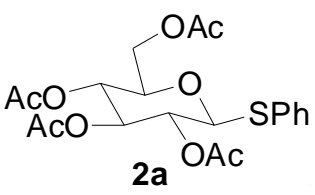

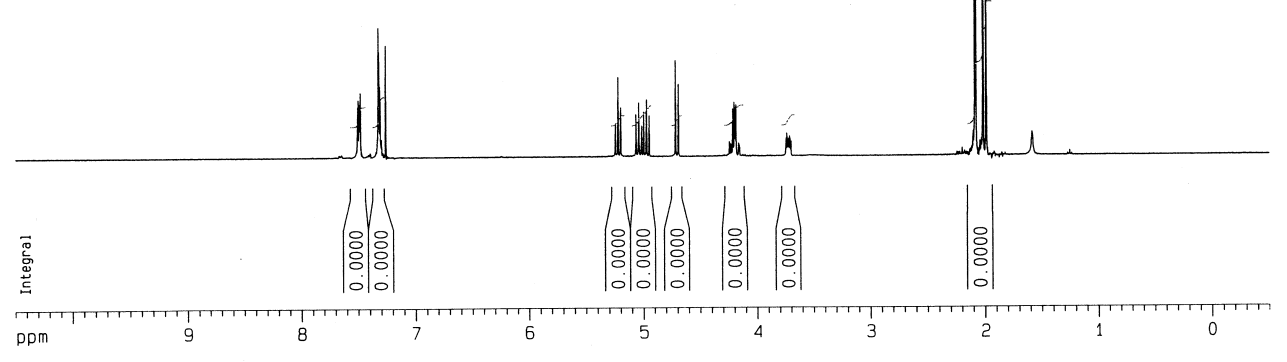

Current Data Parameters

EXPNO

F2 - Acquisition Parameters
Date 20031222

$\begin{array}{rr}20031222 \\ \text { Time } \\ \text { Timste } & 0.19\end{array}$

INSTRUM
PROBHO $5 \mathrm{~mm}$ BBO BB-1H
PROBO

$\begin{array}{lr}\text { PULPROG } & 2930 \\ \text { TO } & 16384 \\ & \text { COC13 }\end{array}$

TD
SOLVENT
NS

$\begin{array}{cc}\text { NS } & 1 \\ \text { DS. } & 0 \\ \text { SWH } & 5995.204\end{array}$

$\begin{array}{ll}5995.204 \mathrm{~Hz} \\ \text { H DRES } & 0.365918 \mathrm{~Hz}\end{array}$

$\begin{array}{lr}A Q & 1.3664756 \mathrm{sec} \\ \mathrm{AG} & 228.1 \\ \mathrm{OW} & 83.400 \mathrm{usec} \\ \text { OW } & 5.50\end{array}$

$\begin{array}{lr}\text { DE } & 6.50 \text { use } \\ \text { TE } & 300.0 \mathrm{~K}\end{array}$

$==== \pm=$ CHANNEL $+1===== \pm=$
NuC1 $1 \mathrm{H}$
$\mathrm{P}_{1} \quad 14.30$ usec

$\begin{array}{lr}\text { P1 } & 14.30 \text { usec } \\ \text { PL1 } & -5.00 \mathrm{~dB} \\ \text { SF01 } & 400.1326008 \mathrm{MHz}\end{array}$

F2 - Processing parameters

$\begin{array}{lc}\text { SI } & 16384 \\ \text { SF } & 400.1300091 \mathrm{MHz} \\ \text { WDW } & \text { EM } \\ \text { SSB } & 0 \\ \text { LB } & 0.10 \mathrm{~Hz} \\ \text { GB } & 0 \\ \text { PC } & 1.00\end{array}$

DMR plot parameters

$\begin{array}{rr}\text { CX } & \text { MMA plot parameters } \\ \mathrm{CY} & 20.00 \mathrm{~cm} \\ & 9.00 \mathrm{~cm}\end{array}$

$10.500 \mathrm{ppm}$
1.
$4201.37 \mathrm{~Hz}$

$\begin{array}{ll}2 \mathrm{P} & -0.500 \mathrm{ppm} \\ 5 & -200.07 \mathrm{~Hz}\end{array}$

PPMCM $\quad 0.55000 \mathrm{ppm} / \mathrm{cm}$

C13 spectrum o

E
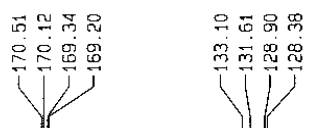

$$
P
$$

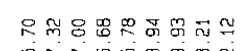

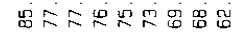

(a)

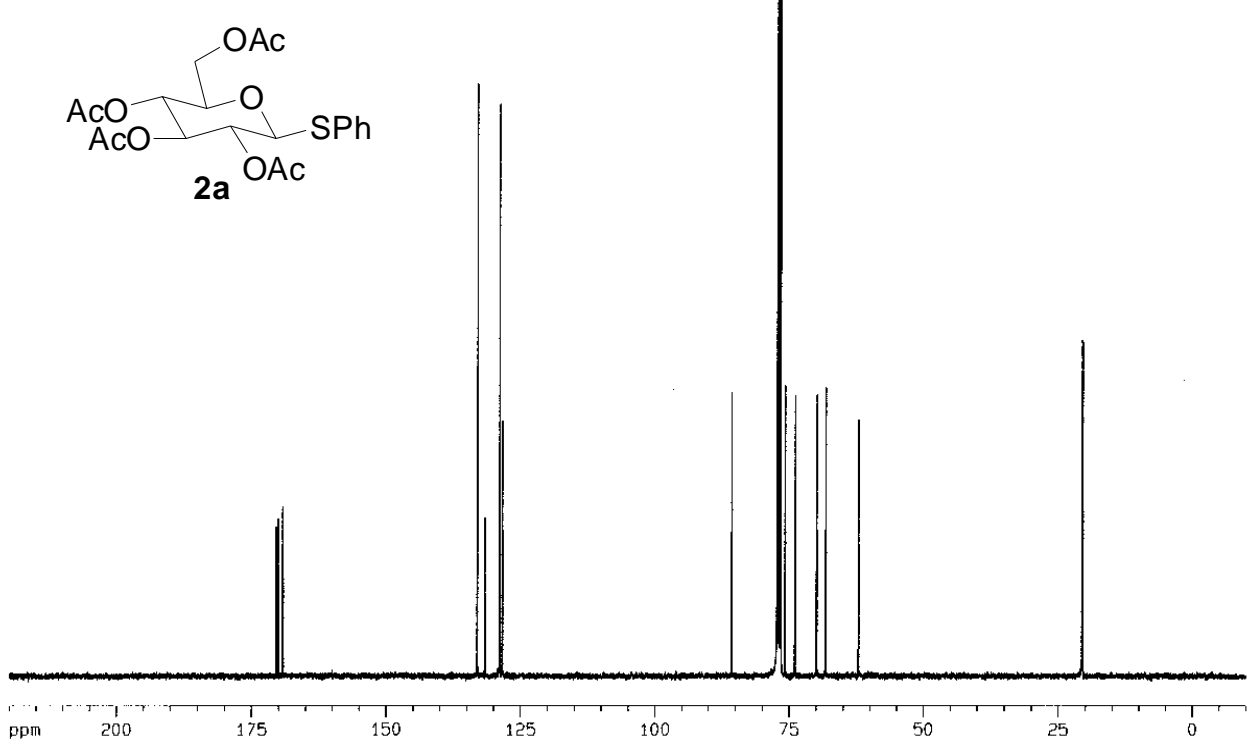

오음

옹요욤

11

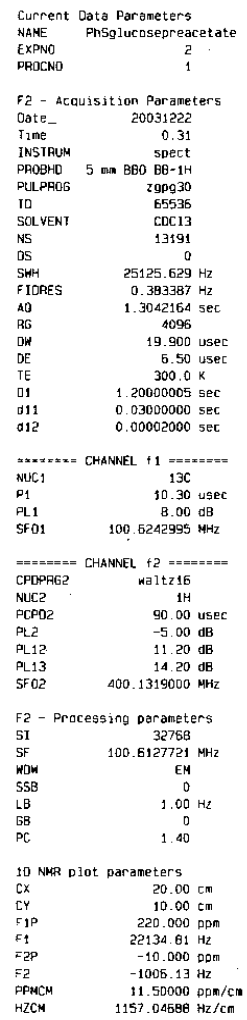


$H$ spectrum of
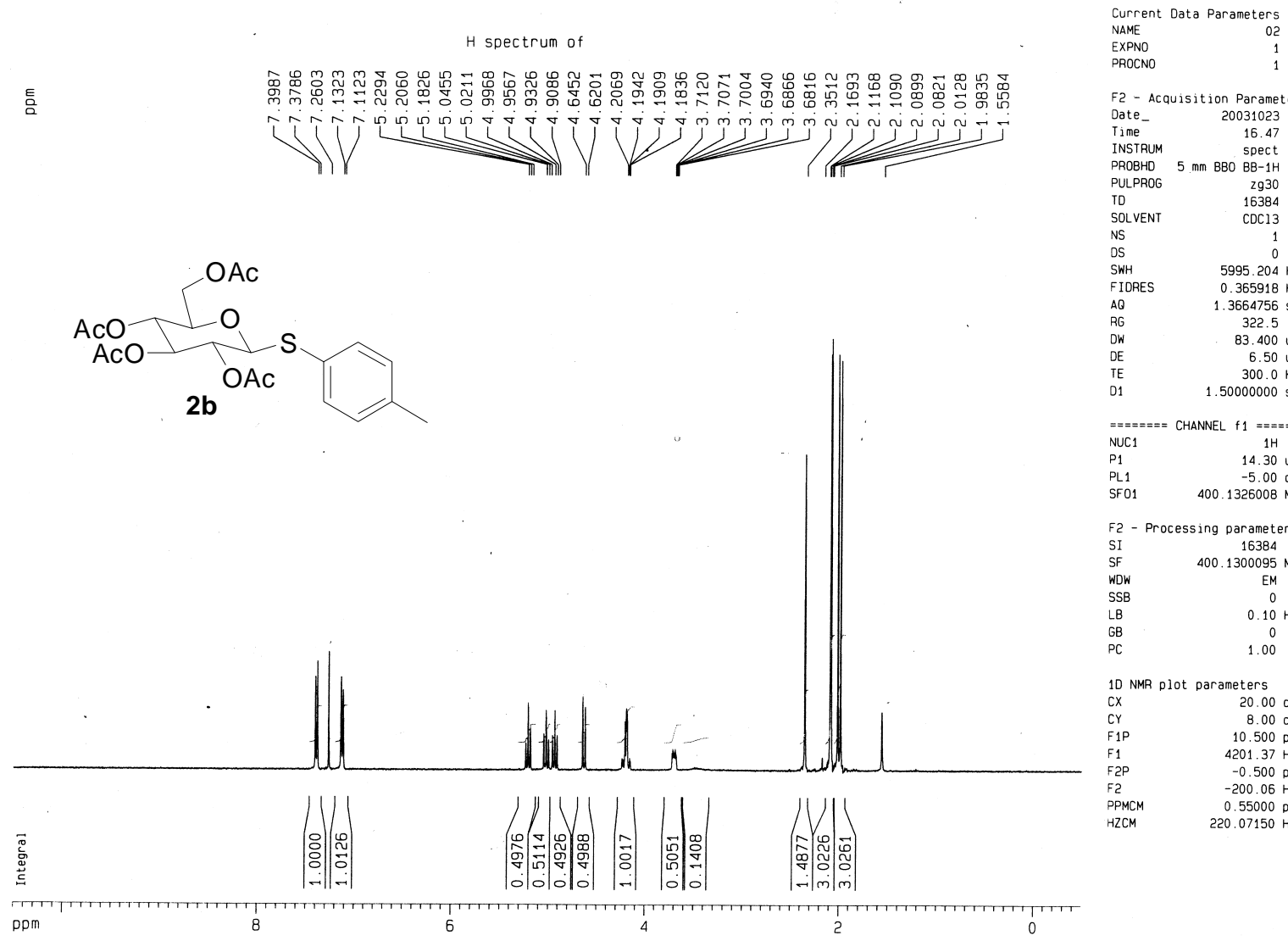

F2 - Acquisition Parameters

Date_ 20031023

$\begin{array}{ll}\text { Time } & 16.47 \\ \text { INSTRUM } & \text { spect }\end{array}$

PROBHD $5 \mathrm{~mm}$ BBO BB-1H

$\begin{array}{lr}\text { PULPROG } & 2930 \\ \text { TD } & 16384\end{array}$

$\begin{array}{lr}\text { SOLVENT } & 16384 \\ \text { NS } & \text { CDC13 } \\ \text { TS } & 1\end{array}$

$\begin{array}{ll}\text { DS } & 0 \\ \text { SWH } & 5995.204 \mathrm{~Hz}\end{array}$

$0.365918 \mathrm{~Hz}$
$1.3664756 \mathrm{seC}$

$\begin{array}{lr}\mathrm{RG} & 322.5 \\ \mathrm{DW} & 83.400 \text { us }\end{array}$

83.400 usec
6.50 usec

$\begin{array}{lr}\text { TE } & 300.0 \mathrm{~K} \\ \text { D1 } & 1.50000000 \mathrm{sec}\end{array}$

$==z===$ CHANNEL $f 1=======$
NUC1 $1 \mathrm{H}$

\begin{tabular}{lr} 
NUC1 & \multicolumn{1}{c}{$1 \mathrm{H}$} \\
P1 & $14.30 \mathrm{use}$ \\
PL1 & $-5.00 \mathrm{~dB}$ \\
SF01 & $400.1326008 \mathrm{MHz}$
\end{tabular}

F2 - Processing parameters

$\begin{array}{lc}\text { SI } & 16384 \\ \text { SF } & 400.1300095 \mathrm{MHZ}\end{array}$

$\begin{array}{lr}\text { WDW } & \text { EM } \\ \text { SSB } & 0\end{array}$

$\begin{array}{lc}\text { LB } & 0.10 \mathrm{HZ} \\ \text { GB } & 0 \\ \text { PC } & 1.00\end{array}$

10 NMR plot parameters

$\begin{array}{lr}\text { CX } & 20.00 \mathrm{~cm} \\ \mathrm{Cr} & 8.00 \mathrm{~cm}\end{array}$ $8.00 \mathrm{~cm}$
$10.500 \mathrm{ppm}$

$\begin{array}{ll}\text { F1 } & 4201.37 \mathrm{~Hz} \\ \text { F2P } & -0.500 \mathrm{ppm} \\ \text { F2 } & -200.06 \mathrm{~Hz}\end{array}$

$\begin{array}{ll}\text { F1 } & 4201.37 \mathrm{~Hz} \\ \text { F2P } & -0.500 \mathrm{ppm} \\ \text { F2 } & -200.06 \mathrm{~Hz}\end{array}$ $\begin{array}{ll}\text { PPMCM } & -200.05000 \mathrm{pDm} / \mathrm{Cm}\end{array}$

C13 spectrum of
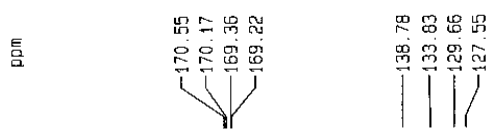<smiles>CC(=O)OCC1OC(=O)OC1C(COC(C)=O)C(=O)O</smiles>

2b<smiles>Cc1ccc(I)cc1</smiles>

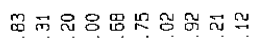

岗

(n)

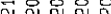

4

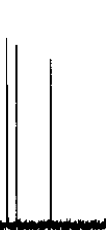

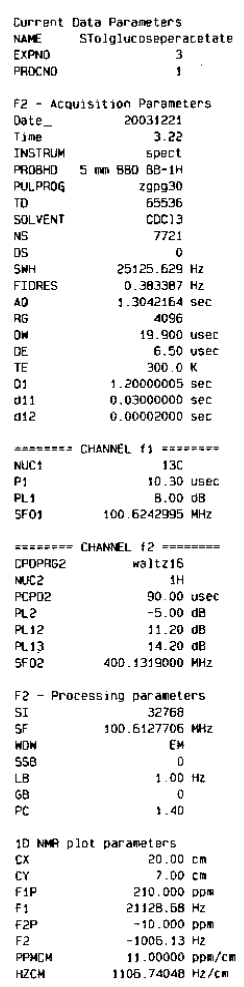




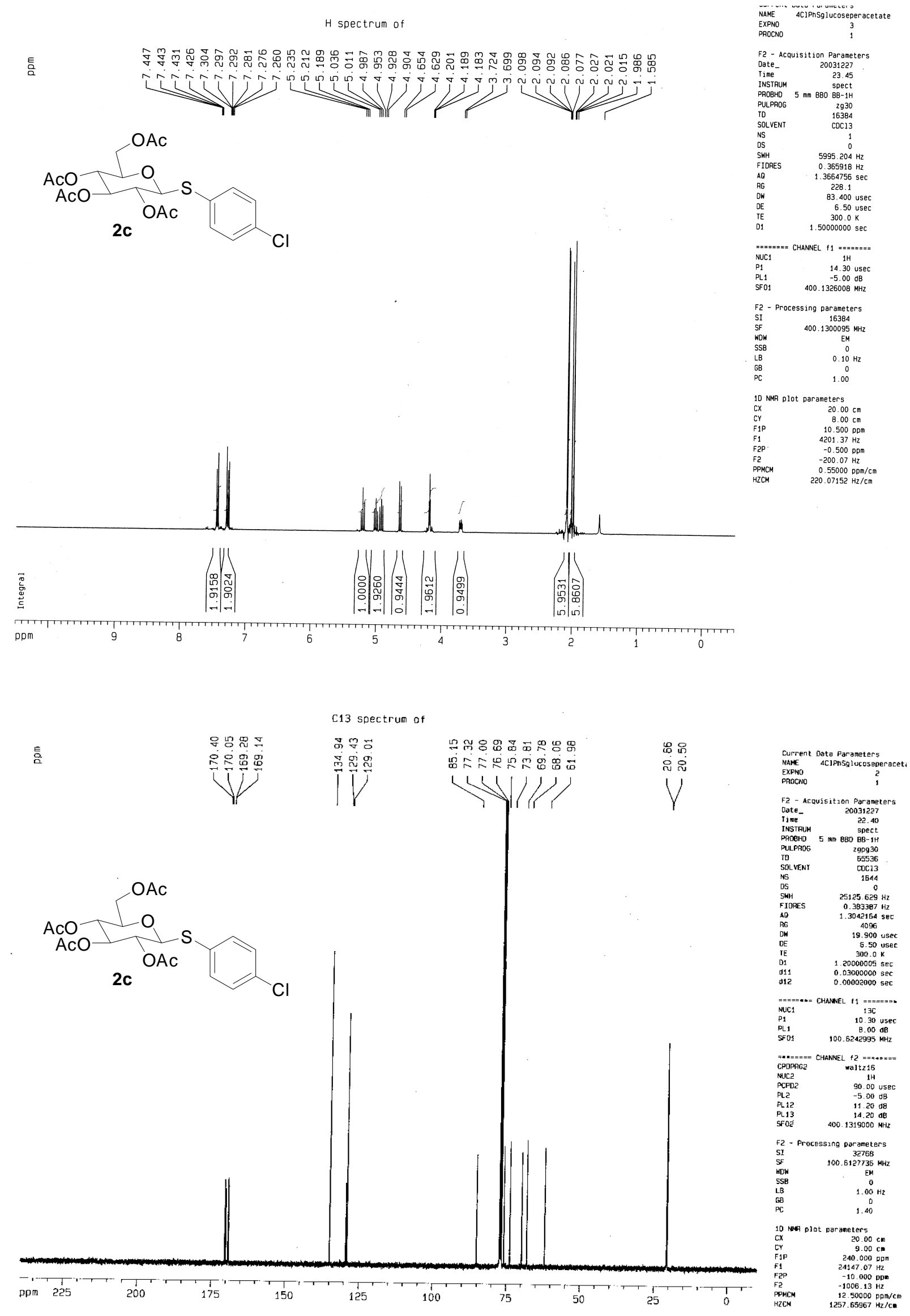



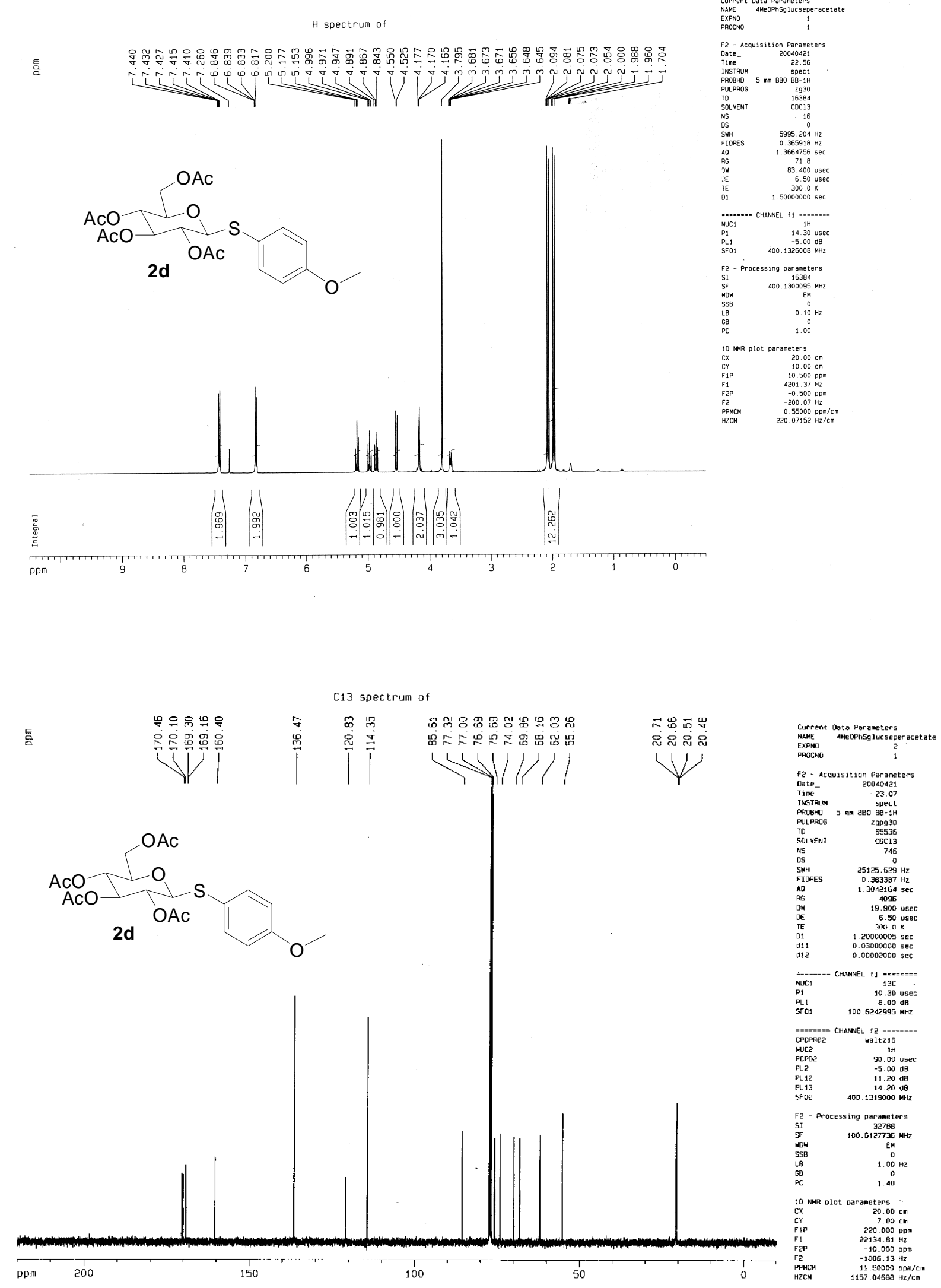
$H$ spectrum of
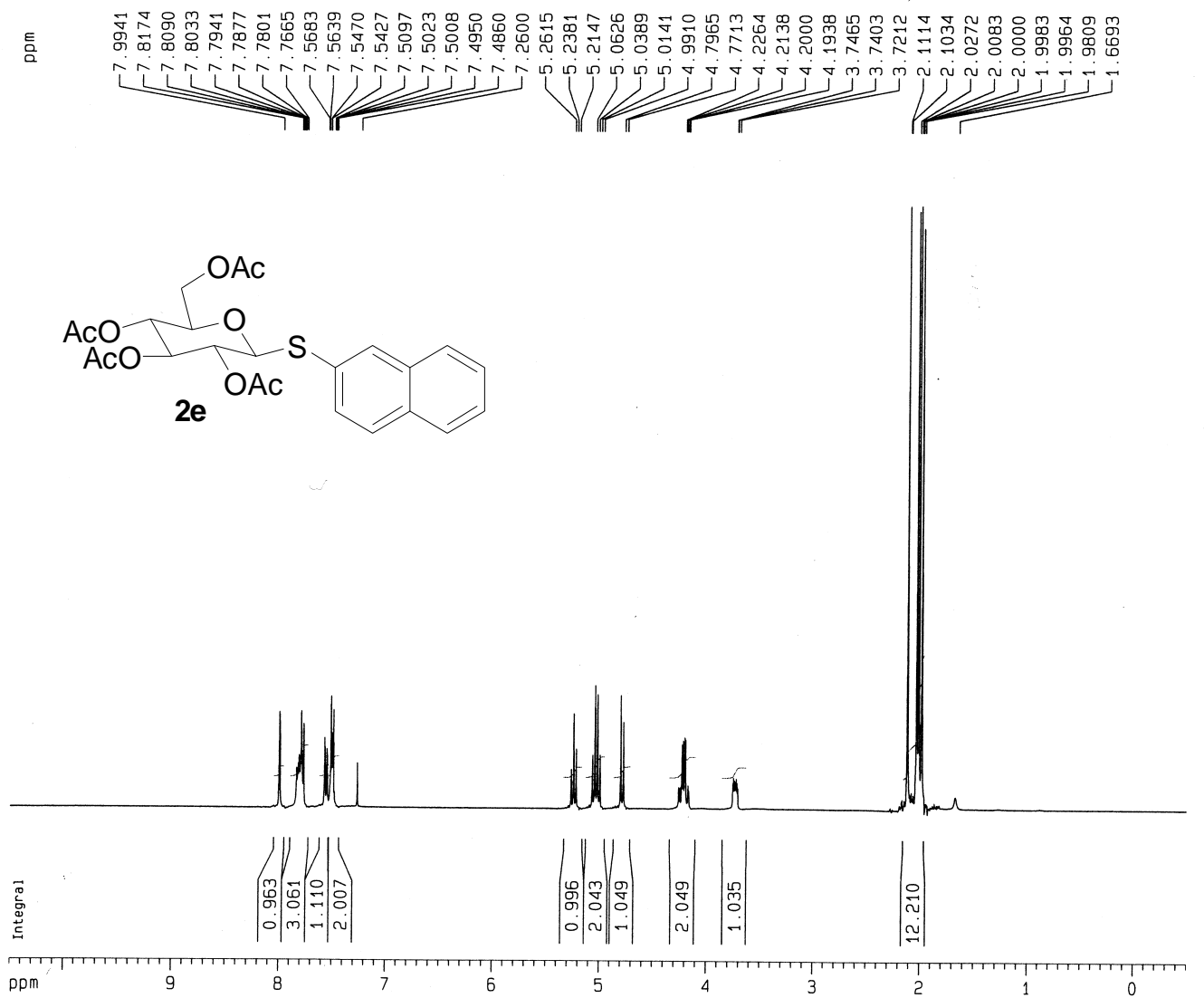

C13 spectrum of

흠

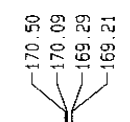

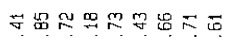

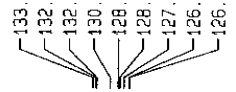

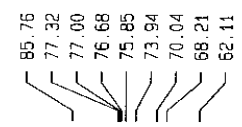

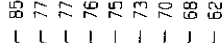

phil?
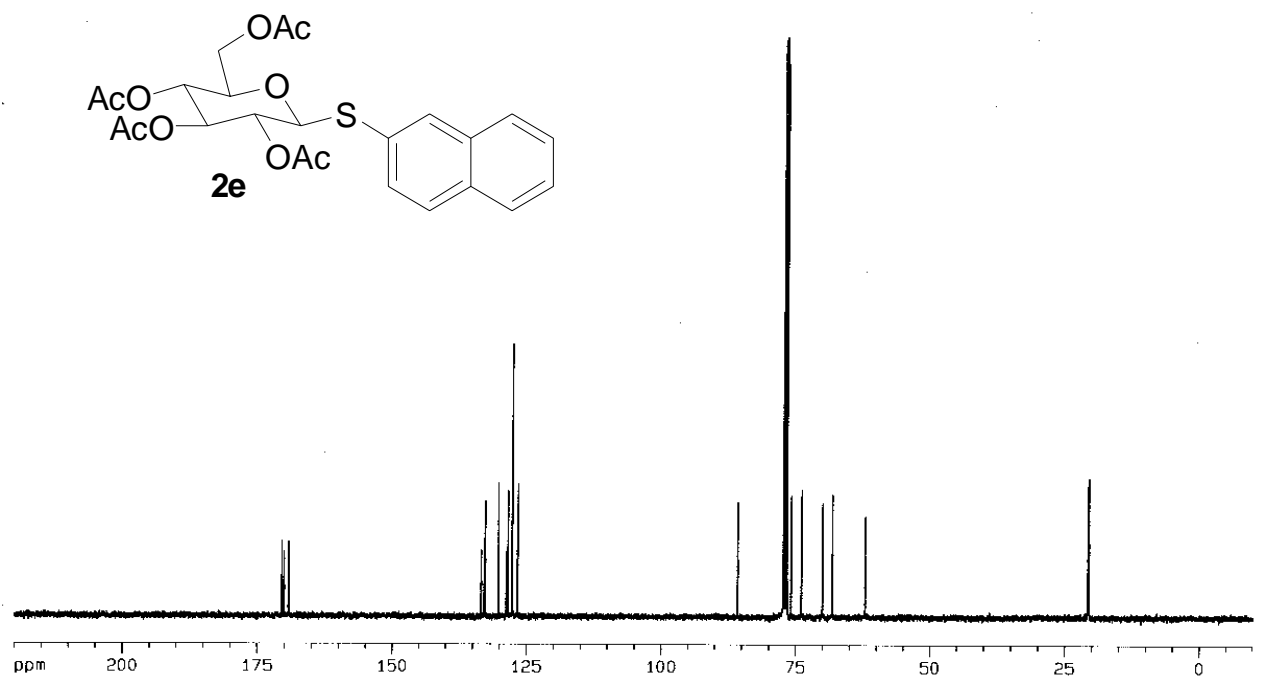

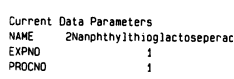

F2 - Accuisition Poraneters
Oote-
Time
Times
21.52

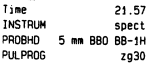

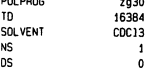

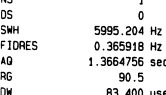

$\begin{array}{lr}\text { OW } & 90.5 \mathrm{usec} \\ \text { OE } & 63.000 \\ \text { TE } & 30.50 \mathrm{usec} \\ \text { TE } & 300.0 \mathrm{~K}\end{array}$

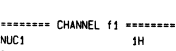

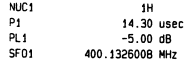

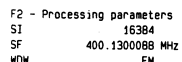

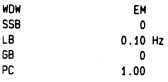

10 MwP plot paraneters
$\mathrm{cx}$
$\mathrm{Cy}$
$\mathrm{Cy}$

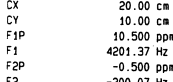

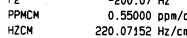




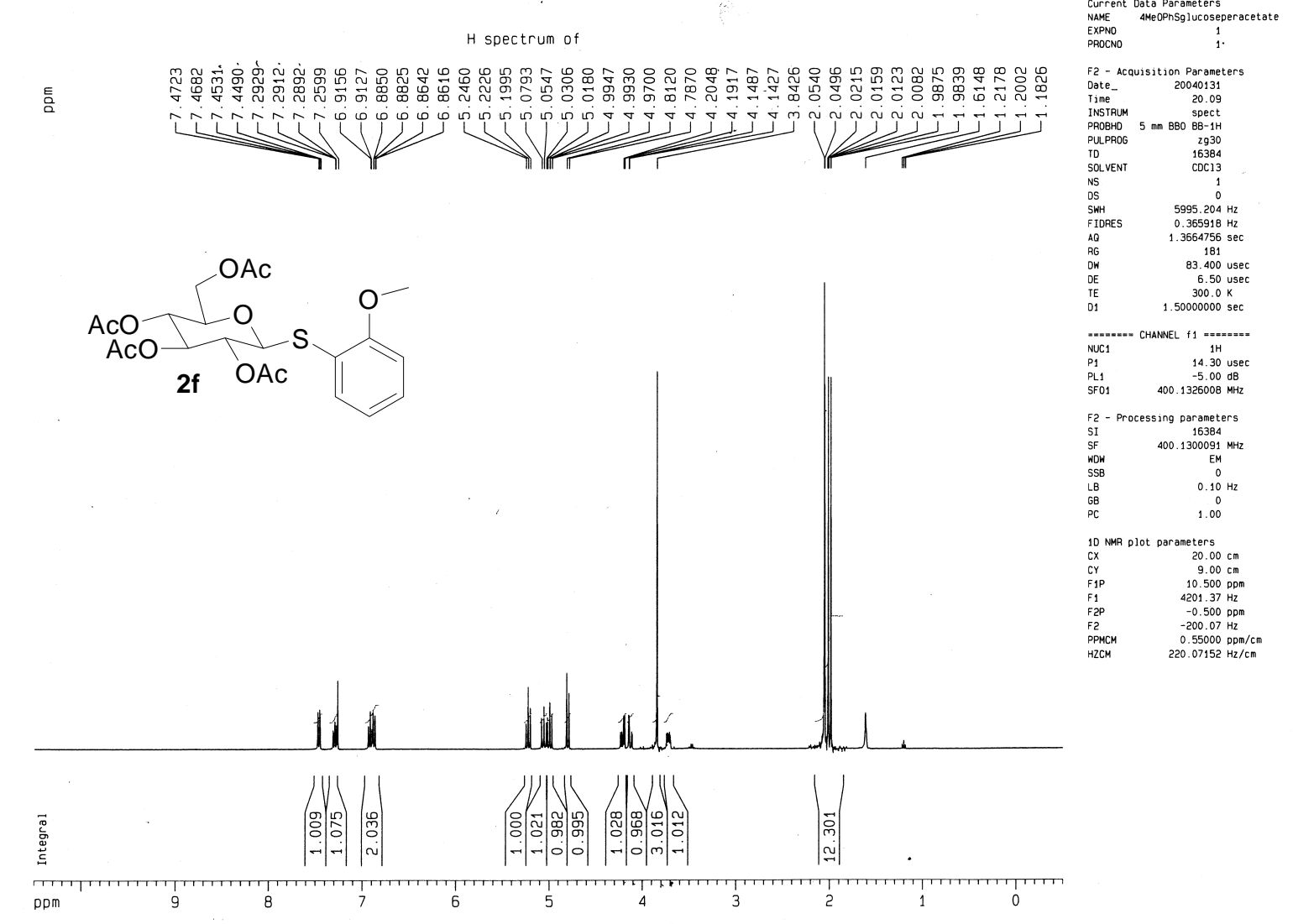

C13 spectrum of

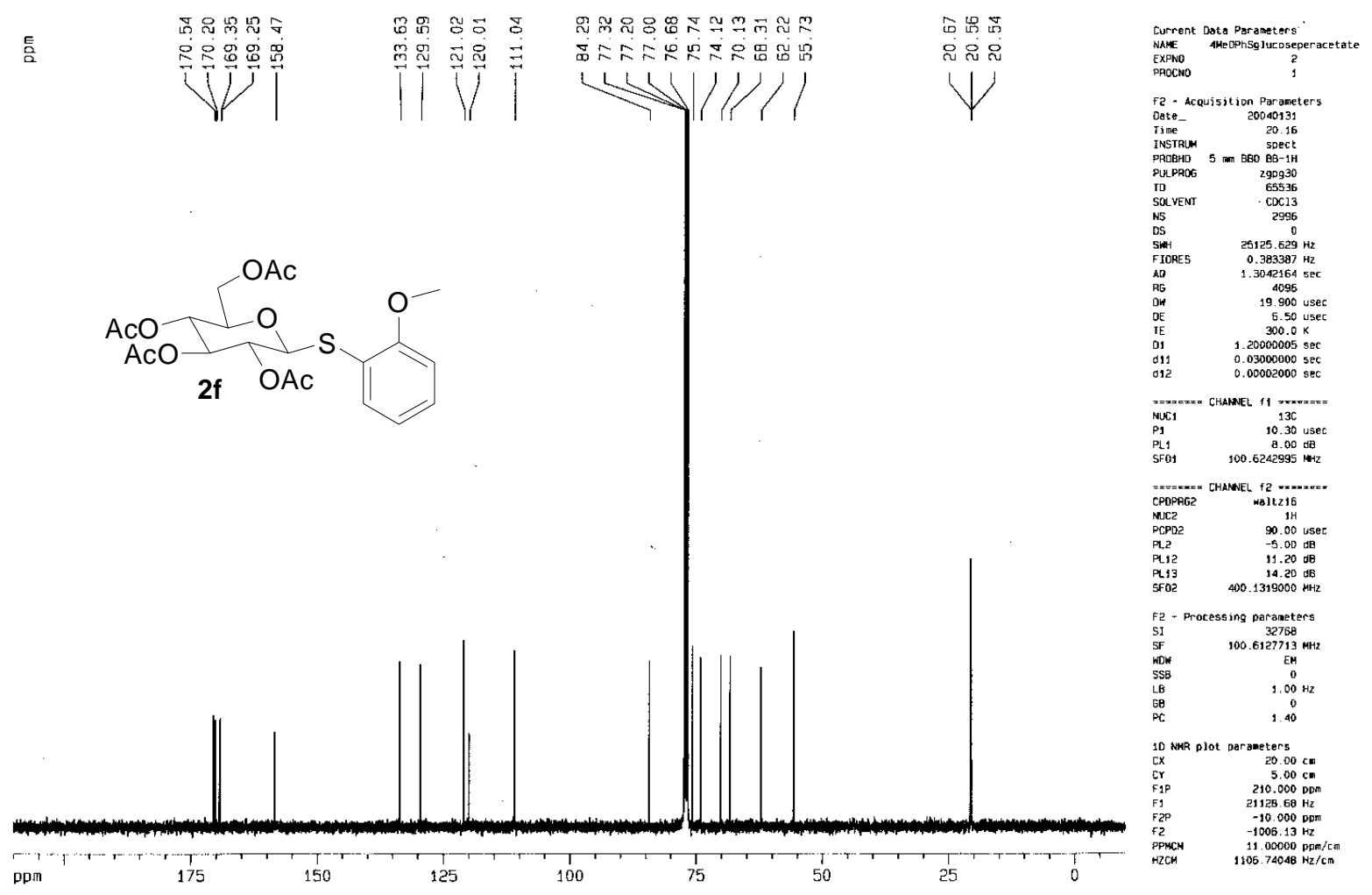




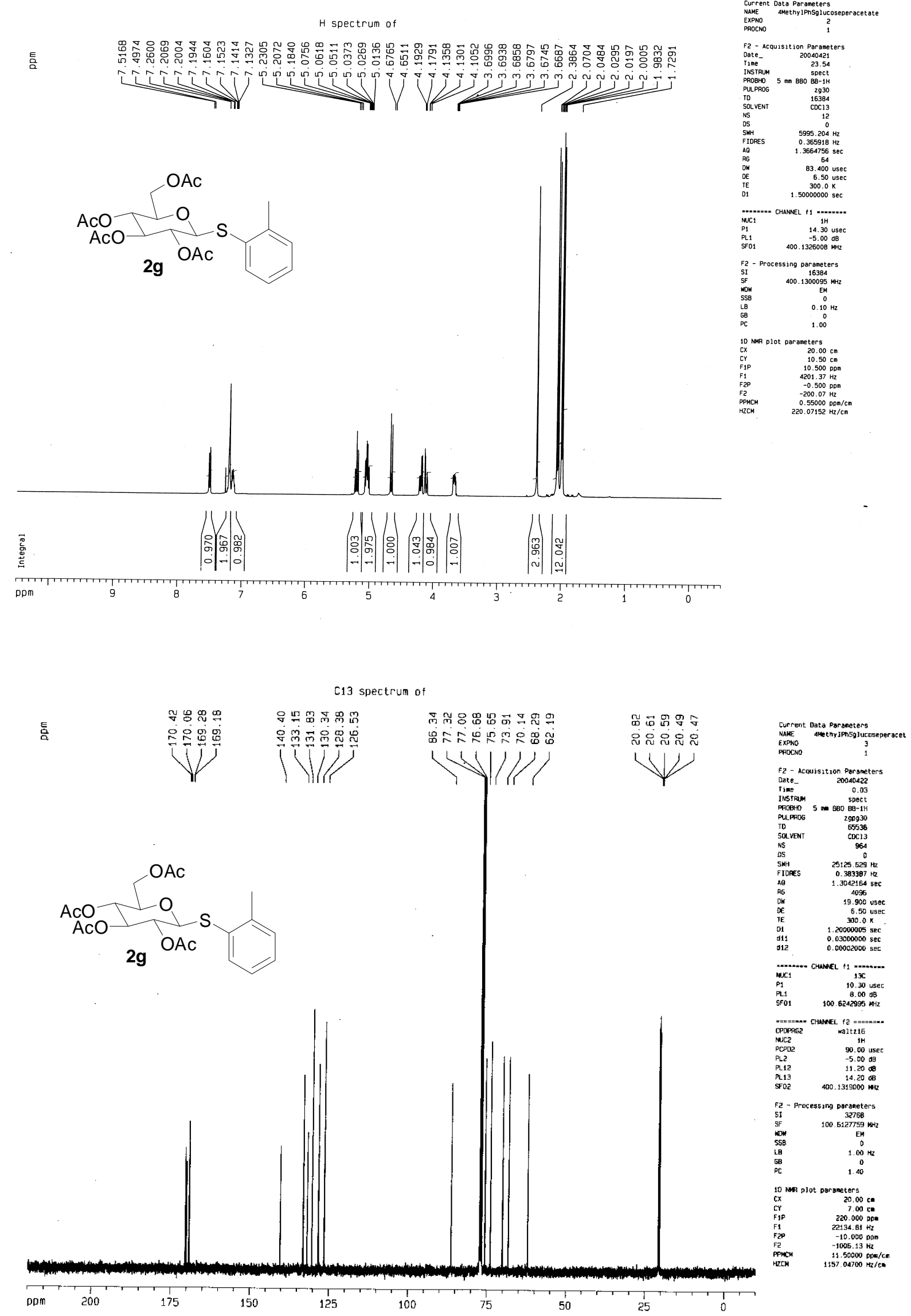




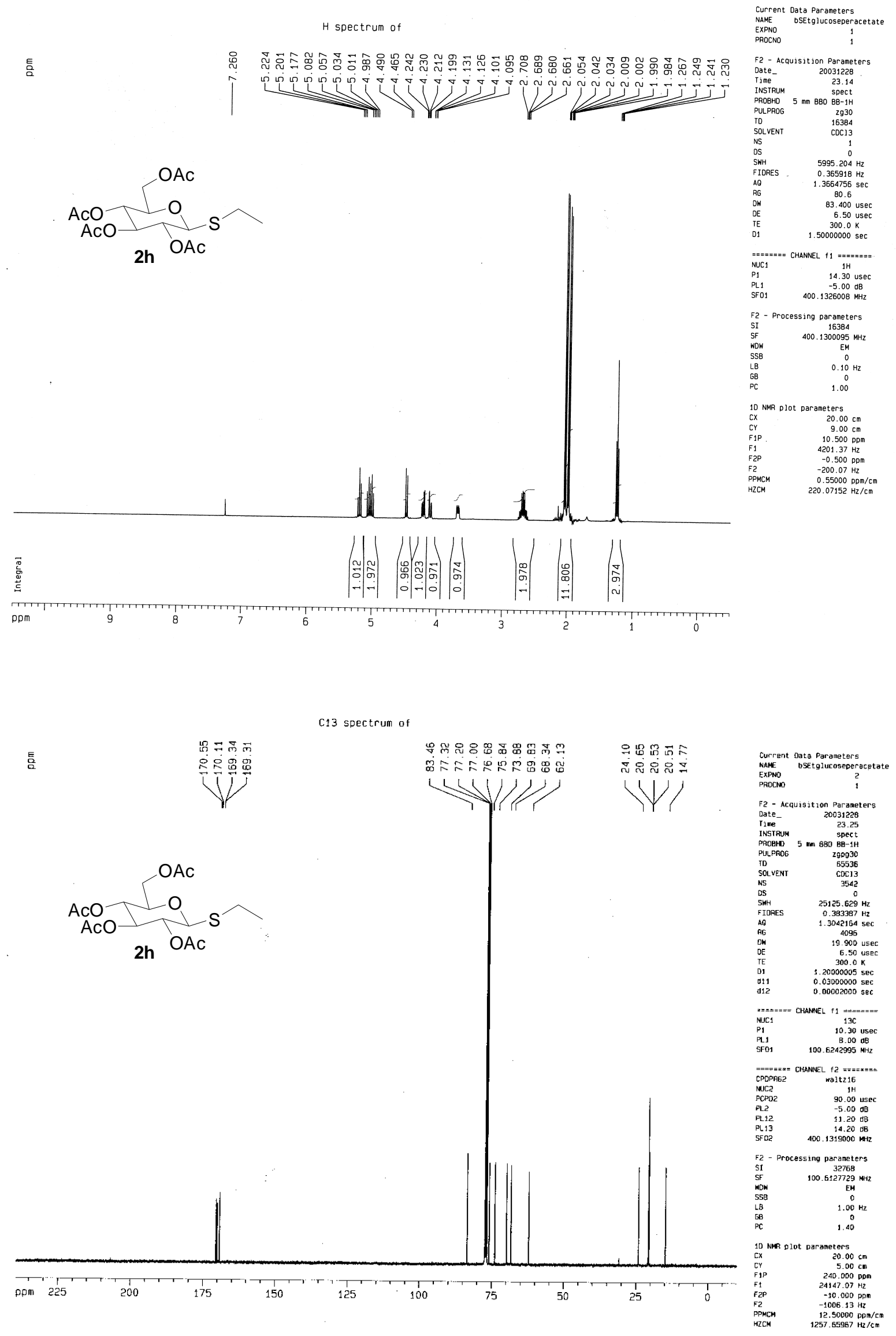




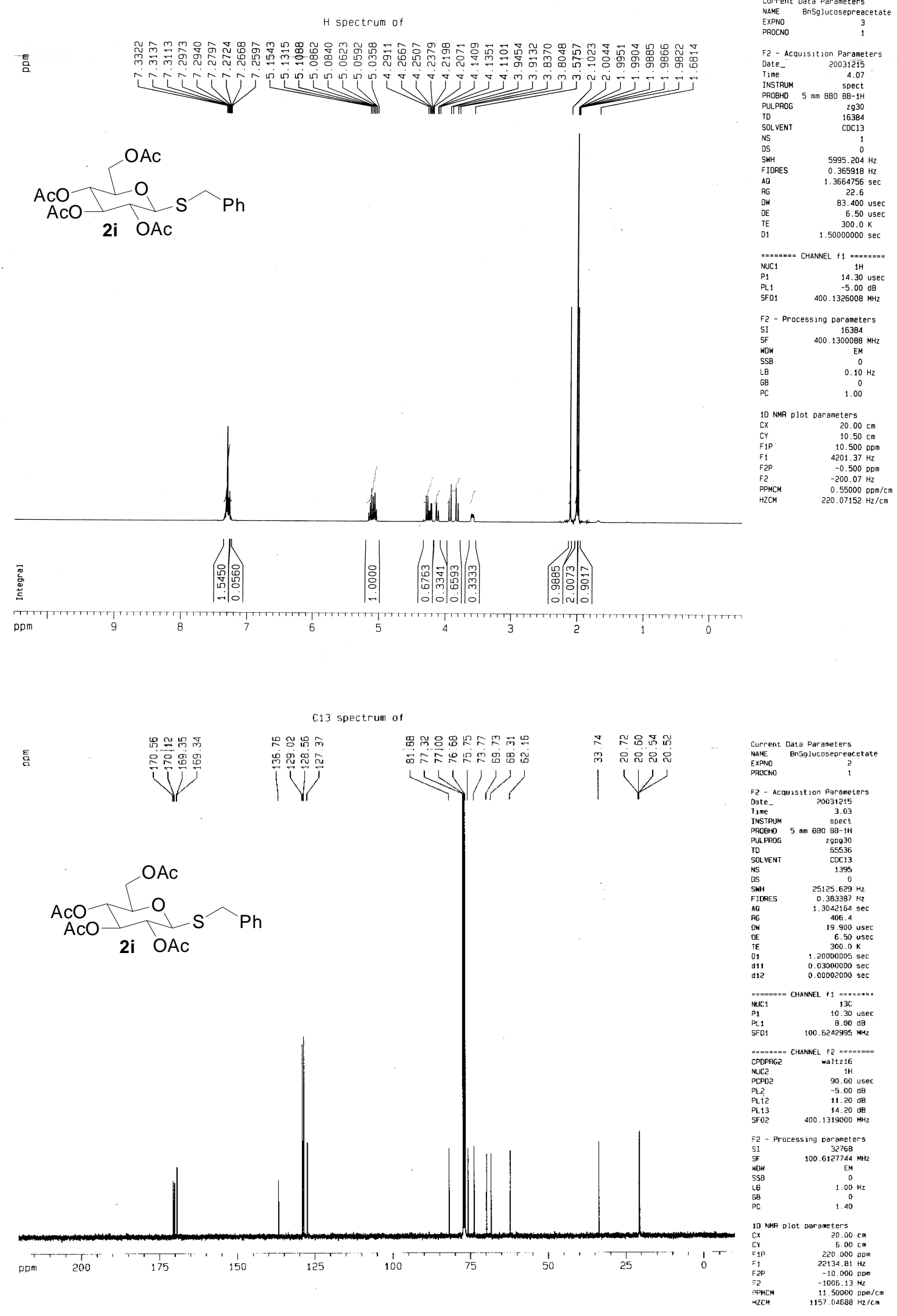




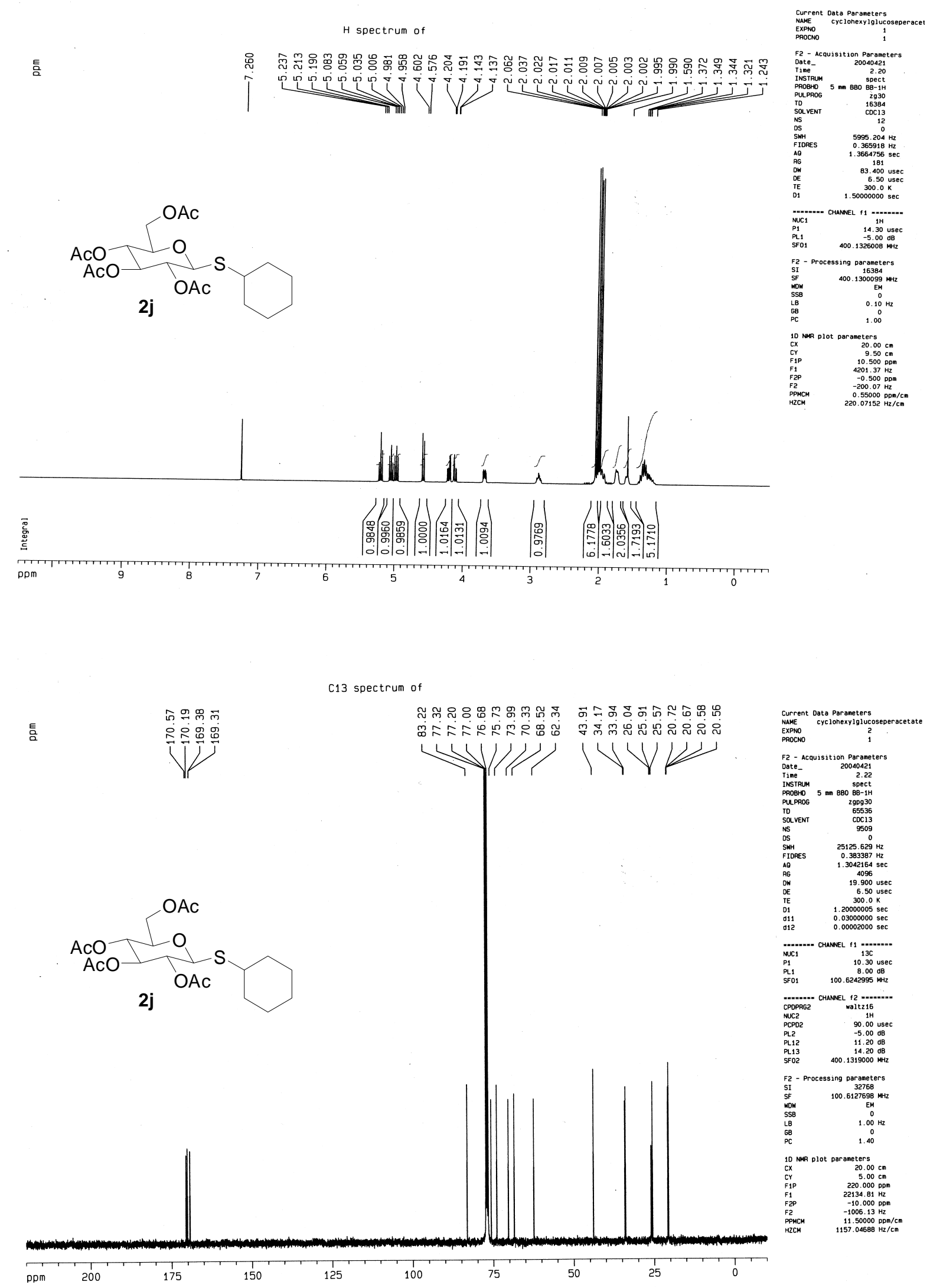



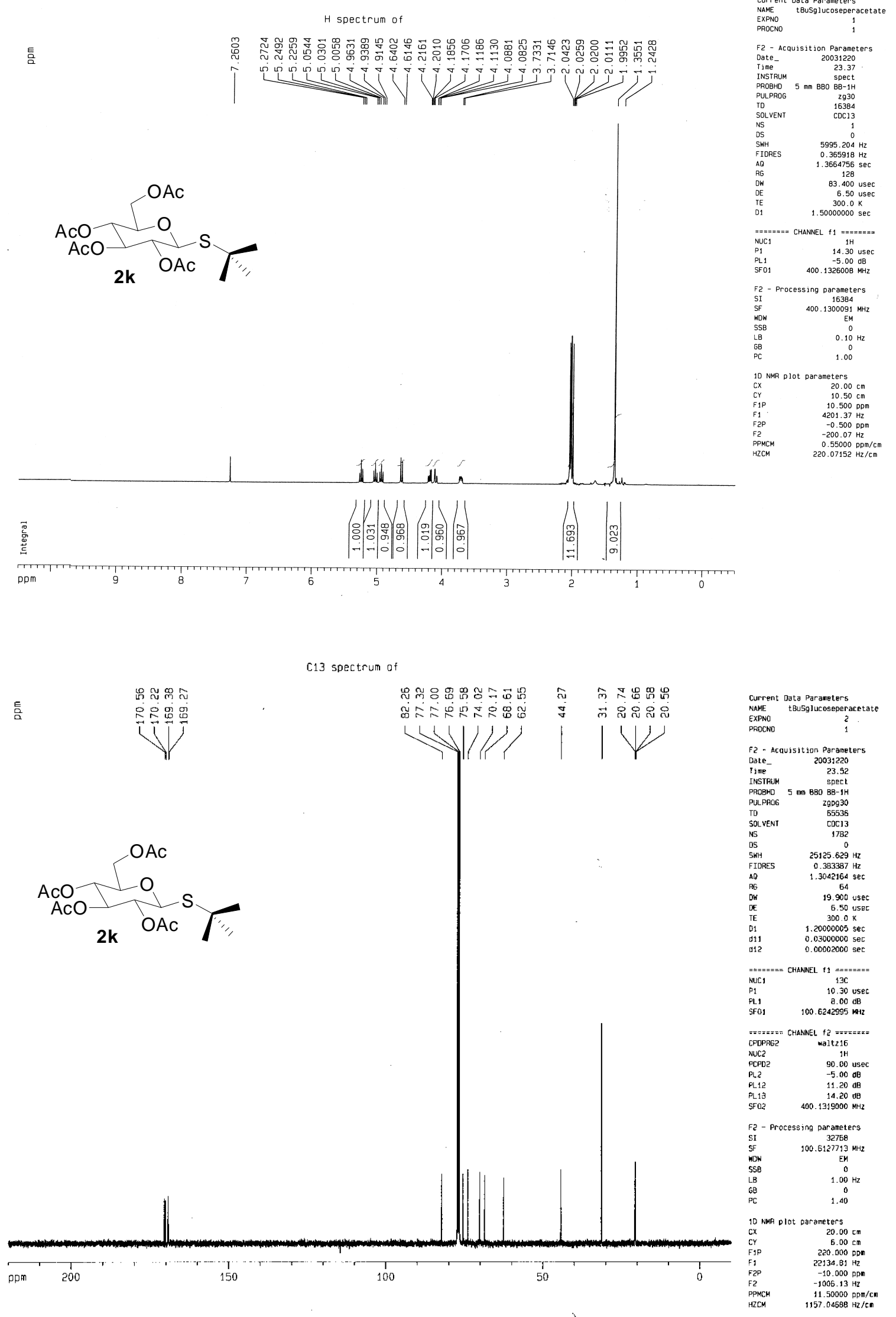


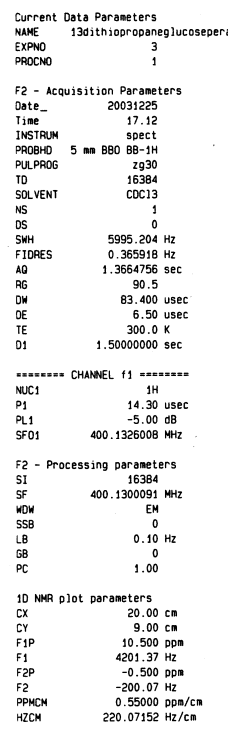

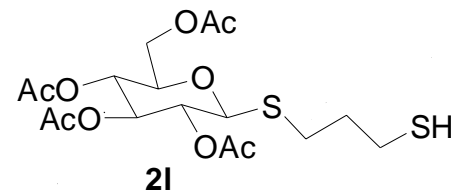

13 spectrum of

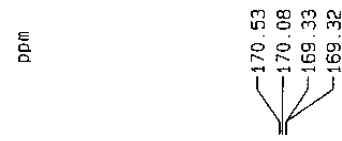

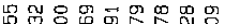

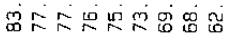
(1)

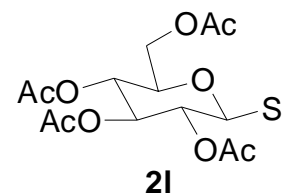
$\mathrm{SH}$
जु भुष

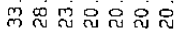

112
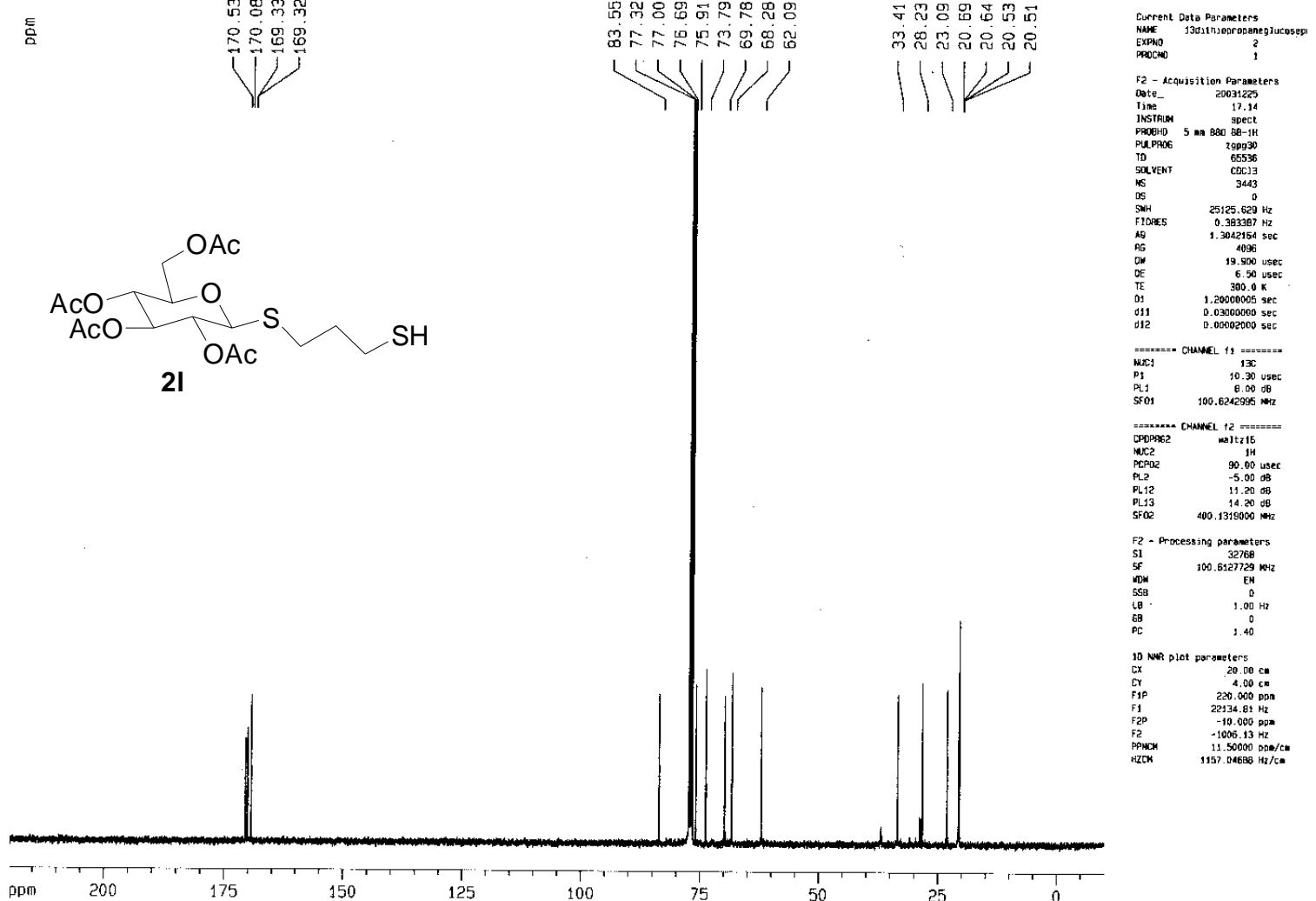


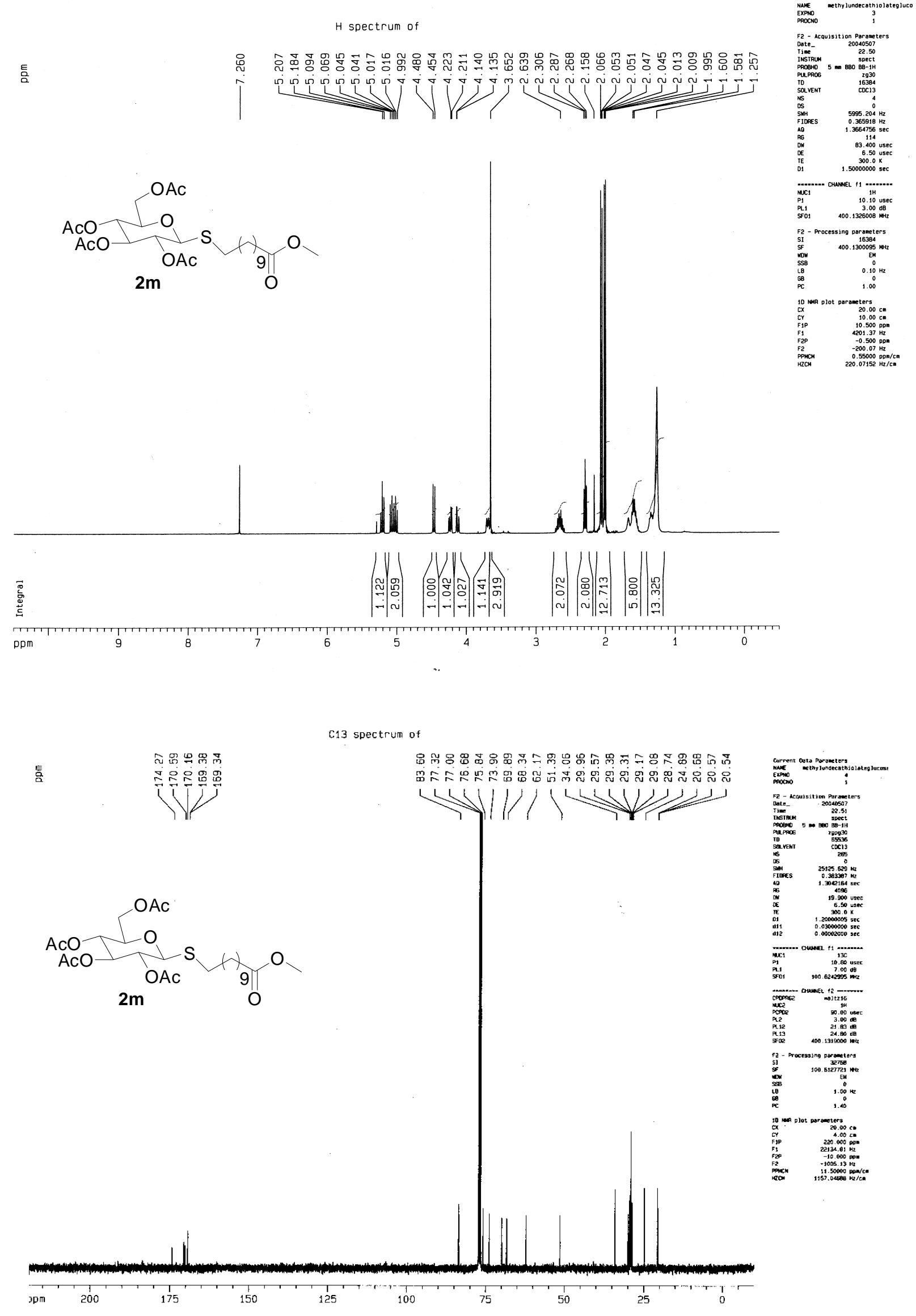




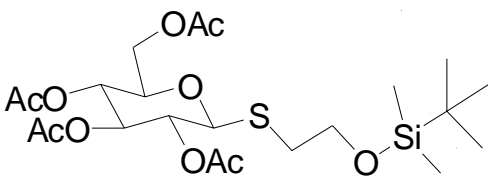

2n

\section{INSTR}

Date_ 20050404

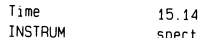

PROBHO $5 \mathrm{~mm}$ BBO BB-1H

$\begin{array}{lr}\text { PULPROG } & 2930 \\ \text { TD } & 16384\end{array}$

$\begin{array}{lr}16384 \\ \text { SOLVENT } & \text { CDC13 } \\ \text { WS } & 10\end{array}$

$\begin{array}{lc}\text { NS } & 10 \\ \text { DS } & 0 \\ \text { SWH } & 5995.204 \mathrm{~Hz}\end{array}$

$\begin{array}{ll} & 5995.204 \mathrm{~Hz} \\ \text { FIDRES } & 0.365918 \mathrm{~Hz}\end{array}$

AQ $\quad 1.3664756 \mathrm{sec}$

$\begin{array}{cc}\text { RG } & 1.3664756 \mathrm{sec} \\ \text { DW } & 362 \\ & 83.400 \mathrm{usec}\end{array}$

6.50 usec

$1.50000000 \mathrm{sec}$

$== \pm=z==$ CHANNEL $f 1=== \pm==3$
NUC 1

$\begin{array}{lc}\text { NUC1 } & 1 \mathrm{H} \\ \text { P1 } & 10.10 \text { usec }\end{array}$

$\begin{array}{lr}\text { PL1 } & 3.00 \mathrm{~dB} \\ \text { SF01 } & 400.1326008\end{array}$

F2 - Processing parameters

$\begin{array}{lr}\text { F2 - Processing parameters } \\ \text { SI } & 16384 \\ \text { SF } & 400.1300095 \mathrm{MHz} \\ \text { WDW } & \mathrm{EM}\end{array}$

$\begin{array}{lc}\text { SSB } & 0 \\ \text { LB } & 0.10 \mathrm{~Hz} \\ \text { GB. } & 0 \\ \text { PC } & 1.00\end{array}$

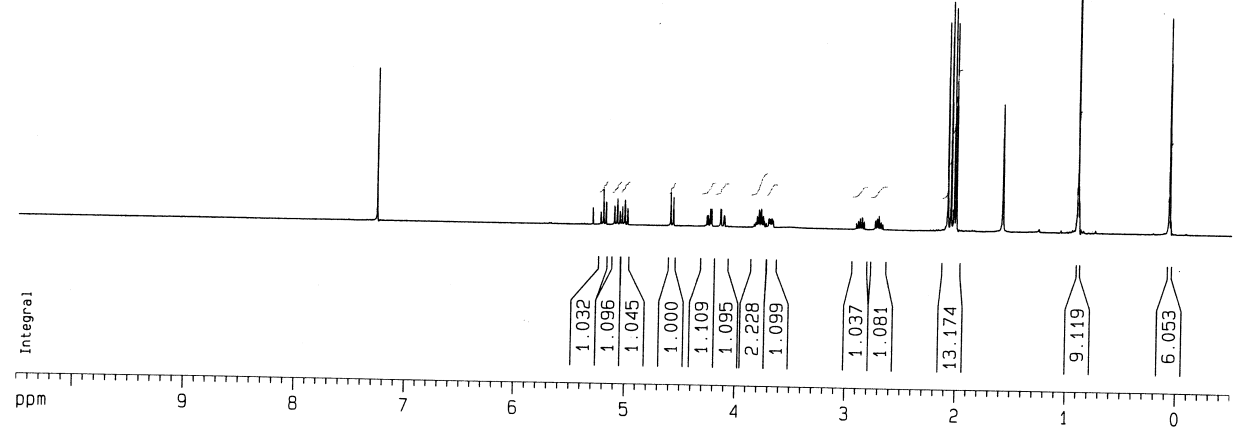

10 NMR plot parameters
CX
CY

$\begin{array}{ll} & 10.00 \mathrm{~cm} \\ F 1 P & 10.500 \mathrm{ppm}\end{array}$

$\begin{array}{ll}\text { F1 } & 4201.37 \mathrm{~Hz} \\ \text { F2P } & -0.500 \mathrm{ppm}\end{array}$

F2 $\quad-200.07 \mathrm{~Hz}$

$\mathrm{HZCM} \quad 220.07152 \mathrm{~Hz} / \mathrm{cm}$

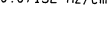

言

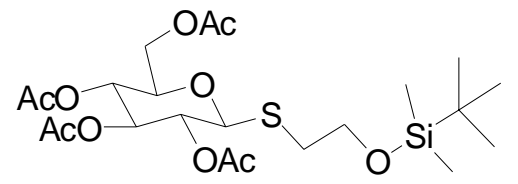

2n

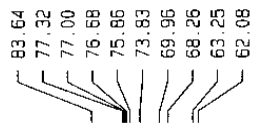

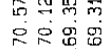

If

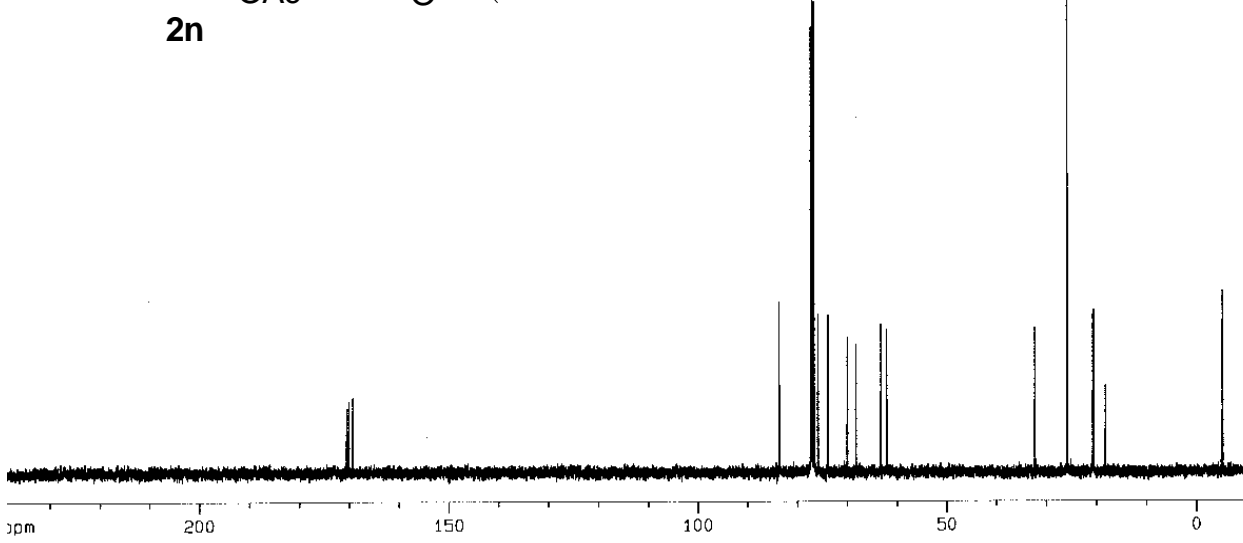

200

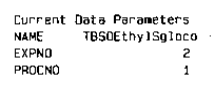

F2 - Acquisition Parameters

Time 14.04
PROBHD

PULLPAOC
TD

TO
SOLVEMT
NS
DS

DS
SWH
FIOHES

a

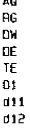

$0.0 .0002000 \mathrm{sec}$

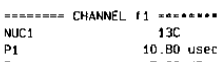

$\begin{array}{lr}\text { PL1 } & 7.00 \mathrm{~dB} \\ \text { SF01 } & \text { 100.6242995 } \mathrm{MHz}\end{array}$

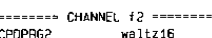

$\begin{array}{lc}\text { CPOPAGE } & \text { waltz16 } \\ \text { NULC } & 1 \mathrm{H} \\ \text { PCPO2 } & 90.00 \text { usec } \\ \text { PL2. } & 3.00 \mathrm{~dB}\end{array}$

$3.00 \mathrm{~dB}$
$\mathrm{PL2}$

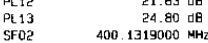

F2 - Pracessing parameters

$\begin{array}{lc}\mathrm{SI} & 32768 \\ \mathrm{SF} & 100.6127729 \\ \text { WDH } & \mathrm{EHz}\end{array}$

WDW
SSB
LA

$\begin{array}{cc} & 1.00 \mathrm{~Hz} \\ \mathrm{~EB} & 0 \\ \mathrm{PC} & 1.40\end{array}$

10 NMA plot paraneters

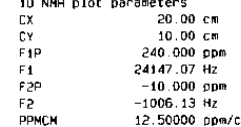


$H$ spectrum of

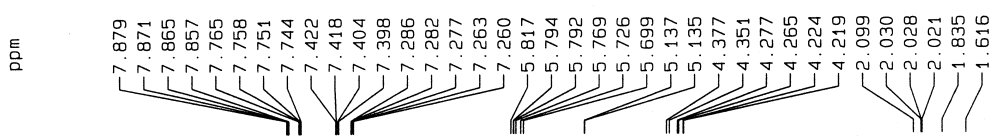
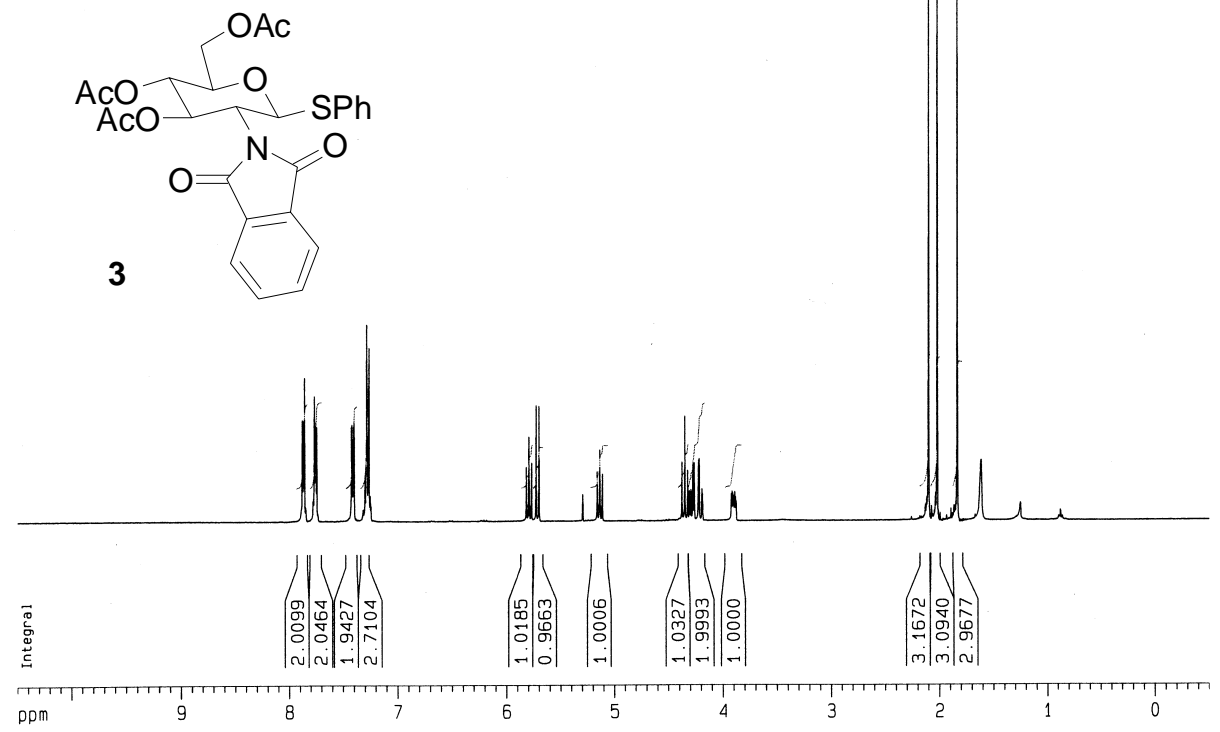

C13 spectrum of
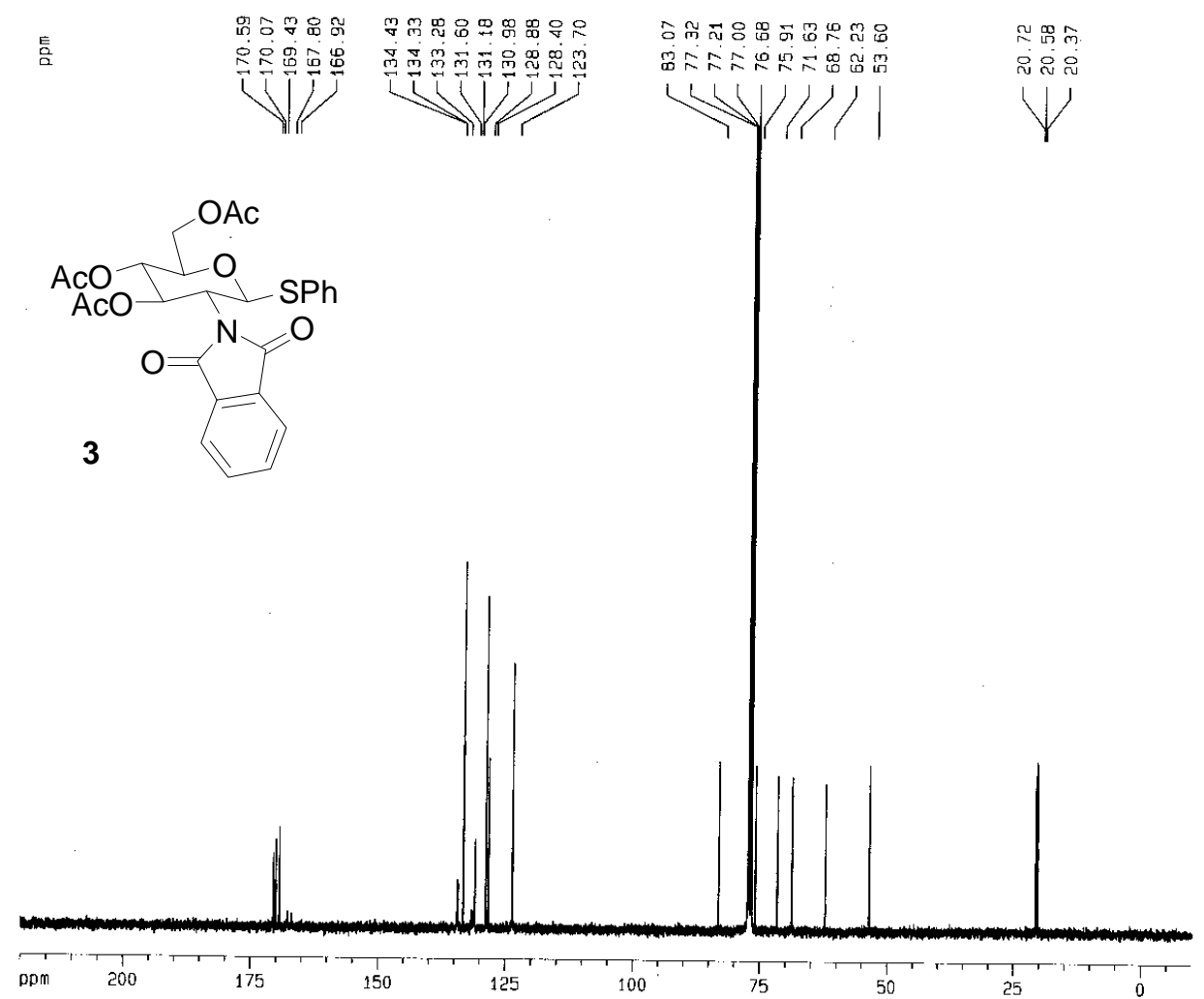

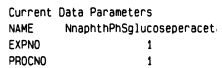

F2 - Acquisition Parameters
Date 20040604

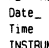

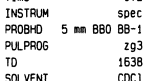

$\begin{array}{lr}\text { SOLVENT } & \text { COC13 } \\ \text { NS } & 8 \\ \text { DS } & 0\end{array}$

$\begin{array}{ll}\text { SWH } & 5995.204 \mathrm{~Hz} \\ \text { FDRES } & 0.365918 \mathrm{~Hz} \\ \text { AO } & 1.3664756 \mathrm{se}\end{array}$

$\begin{array}{lr}\mathrm{AQ} & 1.366456 \mathrm{sec} \\ \mathrm{Ag} & 228.1 \\ \mathrm{PW} & 83.400 \mathrm{usec} \\ \mathrm{OW} & \end{array}$

$\begin{array}{lr}\text { DE } & 6.50 \text { usec } \\ \text { TE } & 300.0 \mathrm{k} \\ \text { D1 } & 1.50000000 \mathrm{sec}\end{array}$

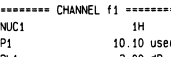

$\begin{array}{lr}\text { PL1 } & 10.10 \text { use } \\ \text { PFo1 } & 3.00 \mathrm{~dB}\end{array}$

F2 - Processing paraneters

$\begin{array}{lc}\text { SI } & 16384 \\ \text { SF } & 400.1300095 \\ \text { MOW } & \text { EM } \\ \text { SSB } & 0\end{array}$

$\begin{array}{ll}\mathrm{LB} & 0.10 \mathrm{~Hz} \\ \mathrm{~GB} & 0 \\ \mathrm{PC} & 1.00\end{array}$

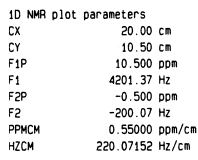


$H$ spectrum of

言

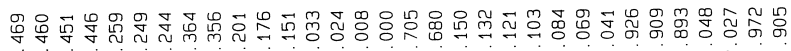
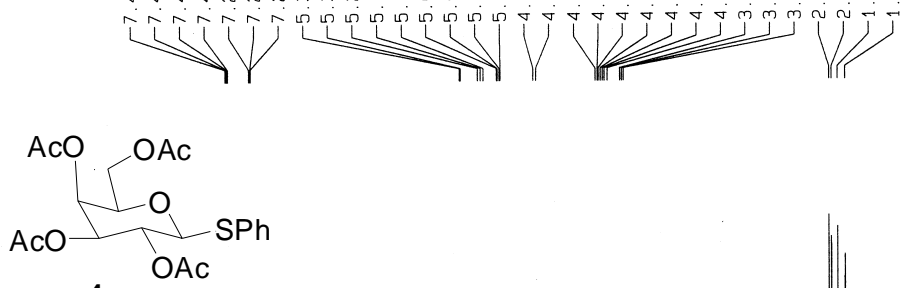

4
Current Data Parameters

NAME PhSgalatopentaAc

PROCNO

F2 - Acquisition Parameters

Date_ 20040421

Time

INSTRUM spect

PULPROG

\begin{tabular}{lr} 
PULPROG & 2930 \\
TD & 16384 \\
\hline
\end{tabular}

$\begin{array}{lr}\text { SOLVENT } & \text { CDC13 } \\ \text { NS } & 16 \\ \text { DS } & 0\end{array}$

SWH $\quad 5995.204 \mathrm{~Hz}$

$\begin{array}{ll}\text { FIDRES } & 0.365918 \mathrm{~Hz} \\ & 1.3664756 \mathrm{sec}\end{array}$

$\begin{array}{lr}\text { AQ } & 1.3664756 \mathrm{sec} \\ \text { RG } & 22.6 \\ \text { DW } & 83.400 \mathrm{usec}\end{array}$ 6.50 use $1.50000000 \mathrm{sec}$ $==z=z==$ CHANNEL $f 1== \pm= \pm==$
NUC1 $\begin{array}{ll}\text { NUC1 } & 1 \mathrm{H} \\ \text { P1 } & 14.30 \mathrm{usec} \\ \text { PL1 } & -5.00 \mathrm{~dB}\end{array}$ $\begin{array}{lr}-5.00 \mathrm{~dB} \\ \text { SF01 } & 400.1326008 \mathrm{MHZ}\end{array}$

F2 - Processing parameters

$\begin{array}{lc}\text { SI } & 16384 \\ \text { SF } & 400.1300029 \mathrm{MHz}\end{array}$

WOW $\quad$ EM

$\begin{array}{lc}\text { SSB } & 0 \\ \text { LB } & 0.10 \mathrm{~Hz} \\ G B & 0\end{array}$

$\begin{array}{ll}\text { GB } & 1.00\end{array}$

10 NMR plot parameters

$\begin{array}{ll}\text { CX } & 20.00 \mathrm{~cm} \\ \mathrm{CY} & 9.00 \mathrm{~cm}\end{array}$

$\begin{array}{ll}\text { CY } & 9.00 \mathrm{~cm} \\ \text { F1P } & 10.500 \mathrm{ppm}\end{array}$

$4201.37 \mathrm{~Hz}$

$-0.500 \mathrm{ppm}$

$-200.06 \mathrm{~Hz}$
$0.55000 \mathrm{ppm} / \mathrm{cm}$

$220.07150 \mathrm{~Hz} / \mathrm{cm}$

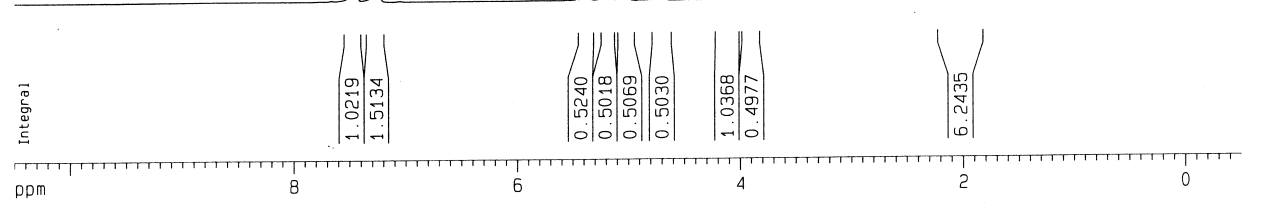

C13 spectrum of

言

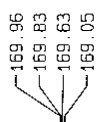

${ }_{O A C}^{A c O}{ }_{O A C h}^{O A c}$

4

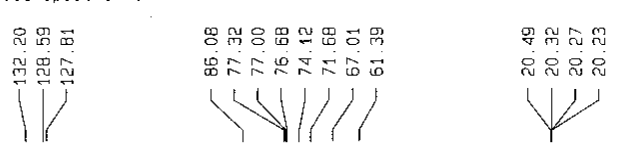

1

NAME
EXPFO
PRO

F2 - Acquisition Parsmeters

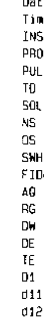

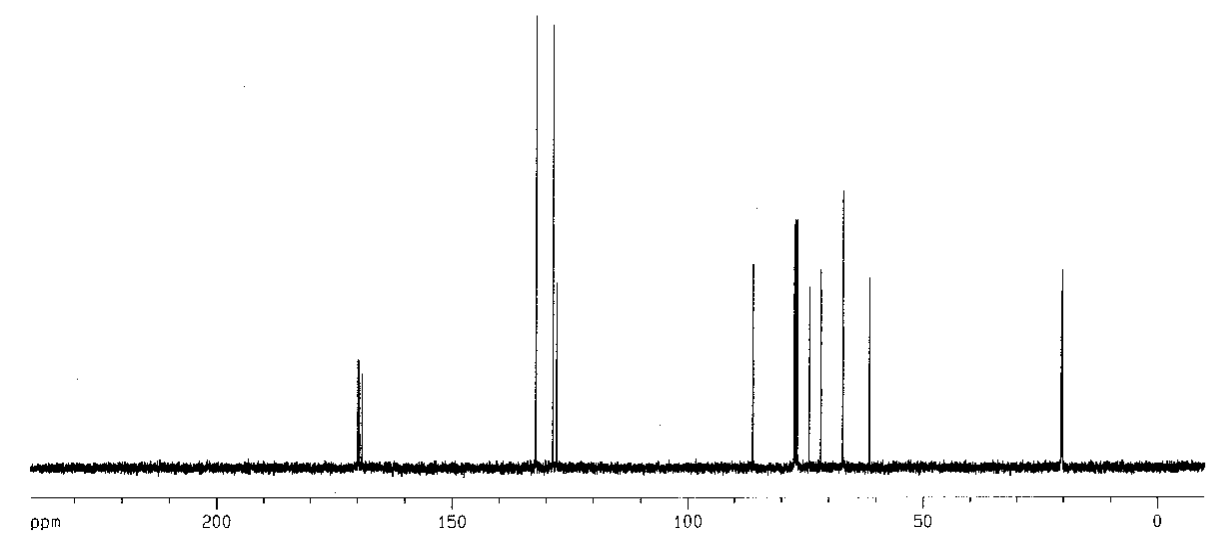

$\quad 16.08$

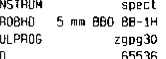

65536
CDOCII VENT

$\begin{array}{cc}\text { JS } & 0 \\ \text { SHH } & 25125.629 \mathrm{~Hz}\end{array}$

$\begin{array}{rr}\text { IDAES } & 0.383397 \mathrm{~Hz} \\ & 1.3042164 \mathrm{sec}\end{array}$

1. 3042164 sec

19.900 usec
5. 50 user
$300.0 \mathrm{~K}$

$300.0 \mathrm{~K}$ 1. $20000005 \mathrm{sec}$ 0.000020005 set

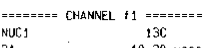

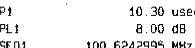
$==x:=\leq=\leq$ CHANWEL $+2=== \pm==$

$\begin{array}{lc}\text { CPDPPG } & \text { walt } 256 \\ \text { NULC2 } & 1 \mathrm{H} \\ \text { OCPDO2 } & 90.00 \mathrm{usec} \\ \text { PL. } & -5.00 \mathrm{~dB} \\ \text { PL12 } & 11.20 \mathrm{~dB} \\ \text { PL19 } & 14.20 \mathrm{~dB}\end{array}$

$400.1919000 \mathrm{MH}$

$\begin{array}{ll}\text { F2 - Processing parameters } \\ \text { SI } & 3776 \mathrm{~g} \\ \text { SF } & \end{array}$

$\begin{array}{lc}\text { SF } & 100.5127936 \mathrm{MHz} \\ \text { WGH } & E M \\ \text { SSB } & 0 \\ \text { LB } & 1.00 \mathrm{~Hz} \\ \text { GB } & 0 \\ \mathrm{PC} & 1.0 \mathrm{C}\end{array}$
10 NHA Dlot parameters
Cx
$20.00 \mathrm{ct}$

$20.00 \mathrm{~cm}$
$8.00 \mathrm{~cm}$ $240.000 \mathrm{pgm}$
$24147.07 \mathrm{~Hz}$

$-10.000 \mathrm{ppen}$ $-1006.13 \mathrm{~Hz}$
$12.50000 \mathrm{ppm} / \mathrm{C}$ $1257.65991 \mathrm{~Hz} / \mathrm{Cm}$ 
$H$ spectrum of
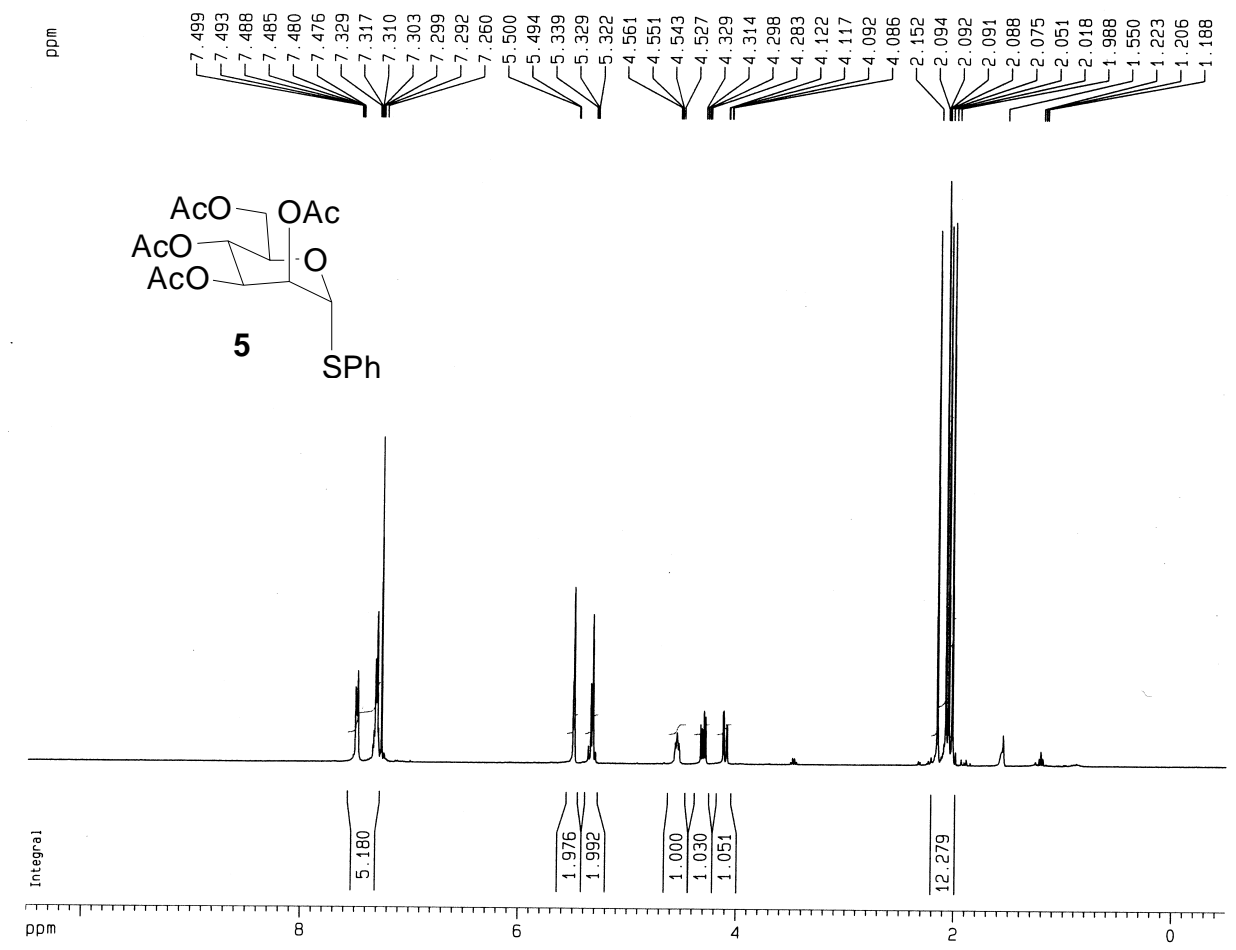

C13 spectrum of

言

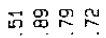

只品嵒

용요욤

要

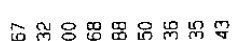

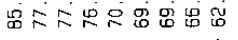

hls
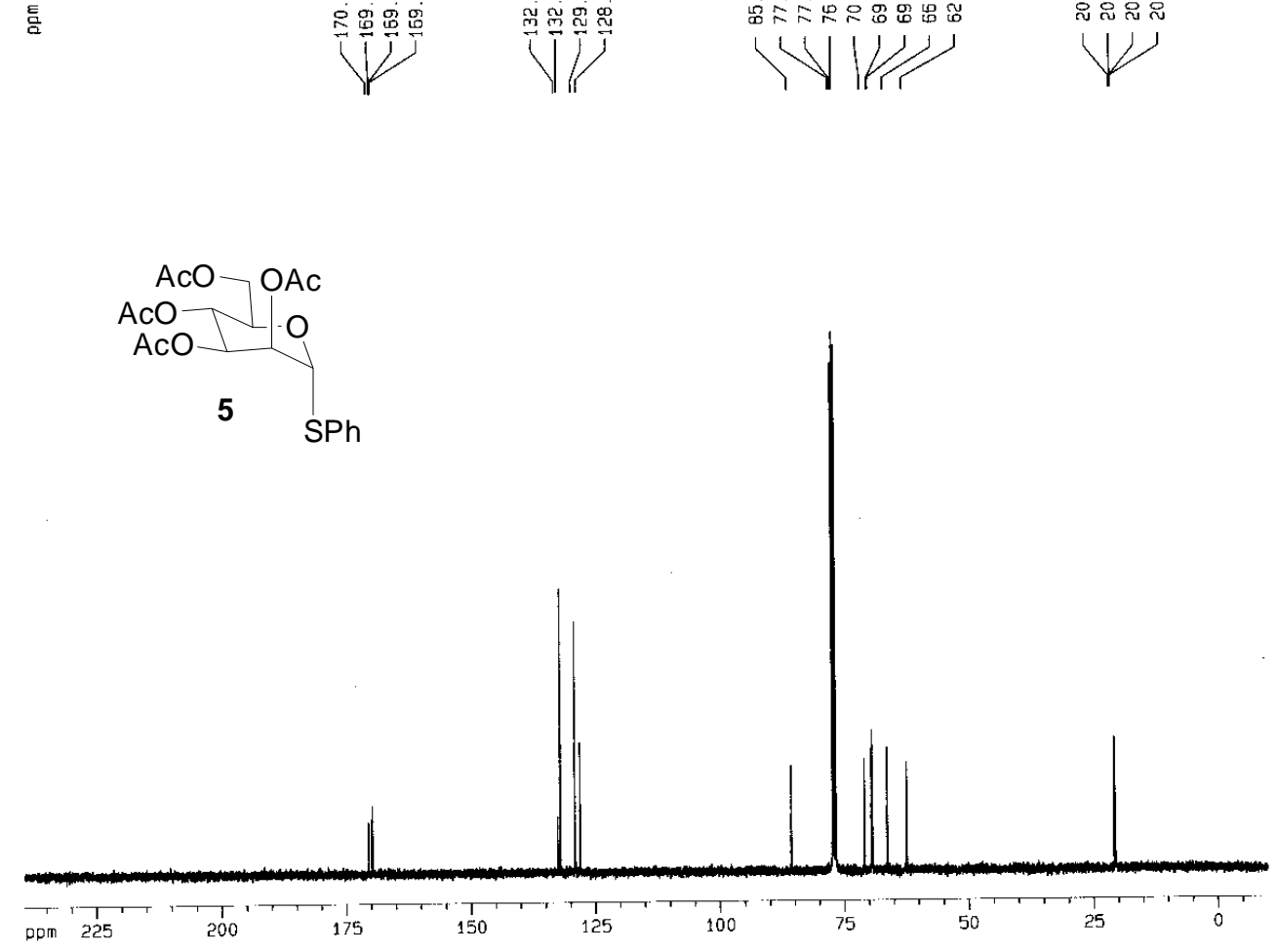

Current Data Parameters
NAME PhSmannoseAC

$\begin{array}{lr}\text { EXME } & \text { PhSmannoseAC } \\ \text { PROCNO } & 1 \\ \text { PRO } & 1\end{array}$

F2 - Acquisition Parameters

$\begin{array}{lr}\text { Date } & 20041122 \\ \text { Time } & 14.12\end{array}$

Time

spect
PROBHD $5 \mathrm{~mm}$ BBO BB-1H

$\begin{array}{lr}\text { PULPROG } & 2930 \\ \text { TD } & 16384\end{array}$

SOLVENT

16
DS

$\begin{array}{ll}\text { SWH } & 5995.204 \mathrm{~Hz} \\ \text { IDDRES } & 0.365918 \mathrm{~Hz}\end{array}$

$\begin{array}{lc}\text { AQ } & 1.3664756 \mathrm{sec} \\ \text { RG } & 406.4\end{array}$

$\begin{array}{lr}\text { RG } & 406.4 \\ \text { DW } & 83.400 \text { usec } \\ \text { DE } & 6.50 \text { usec }\end{array}$ 1. $50000000 \mathrm{sec}$

$z==z===$ CHANNEL $f 1===== \pm=$
NUC1

10.10 usec
$P_{1}$

$\begin{array}{lr}\text { PL1 } & 3.00 \mathrm{~dB} \\ \text { SF01 } & 400.1326008 \mathrm{MHz}\end{array}$

F2 - Processing parameters

$\begin{array}{lc}\text { SI } & 16384 \\ \text { SF } & 400.1300091 \mathrm{MHz} \\ \text { WDW } & \text { EM } \\ \text { SSB } & 0\end{array}$

$\begin{array}{lc}\text { LB } & 0 \\ G B & 0.10 \mathrm{~Hz}\end{array}$

10 NMR plot parameters
20.00
CX

$\begin{array}{ll}\text { CX } & 20.00 \mathrm{~cm} \\ \mathrm{CY} & 10.00 \mathrm{~cm}\end{array}$

$\begin{array}{lr}\text { F1P } & 10.500 \mathrm{ppm} \\ \text { F1 } & 4201.37 \mathrm{~Hz}\end{array}$

$-0.500 \mathrm{ppm}$

$0.55000 \mathrm{ppm} / \mathrm{cm}$
$220.07150 \mathrm{~Hz} / \mathrm{cm}$
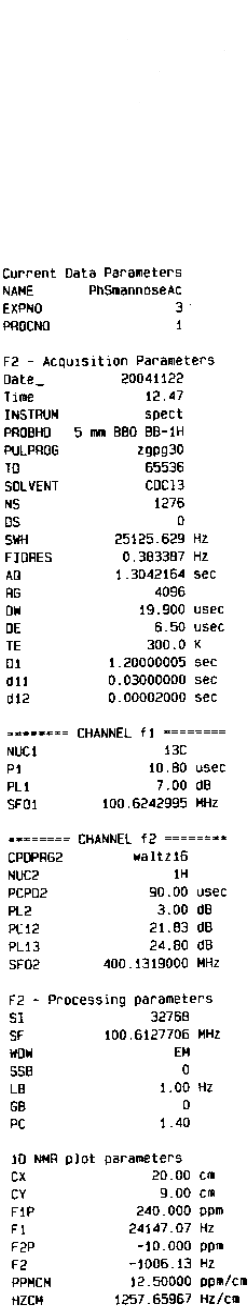

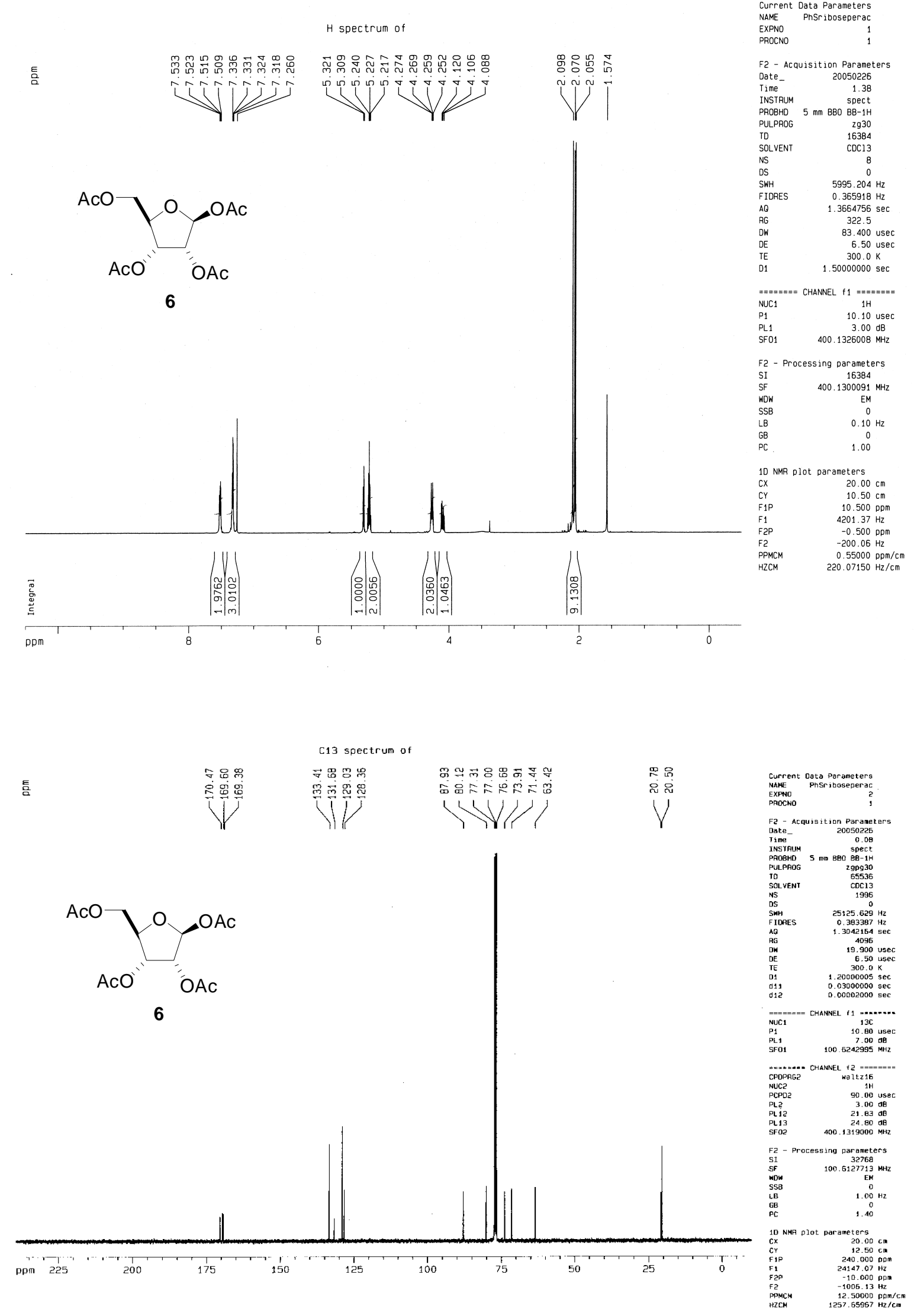


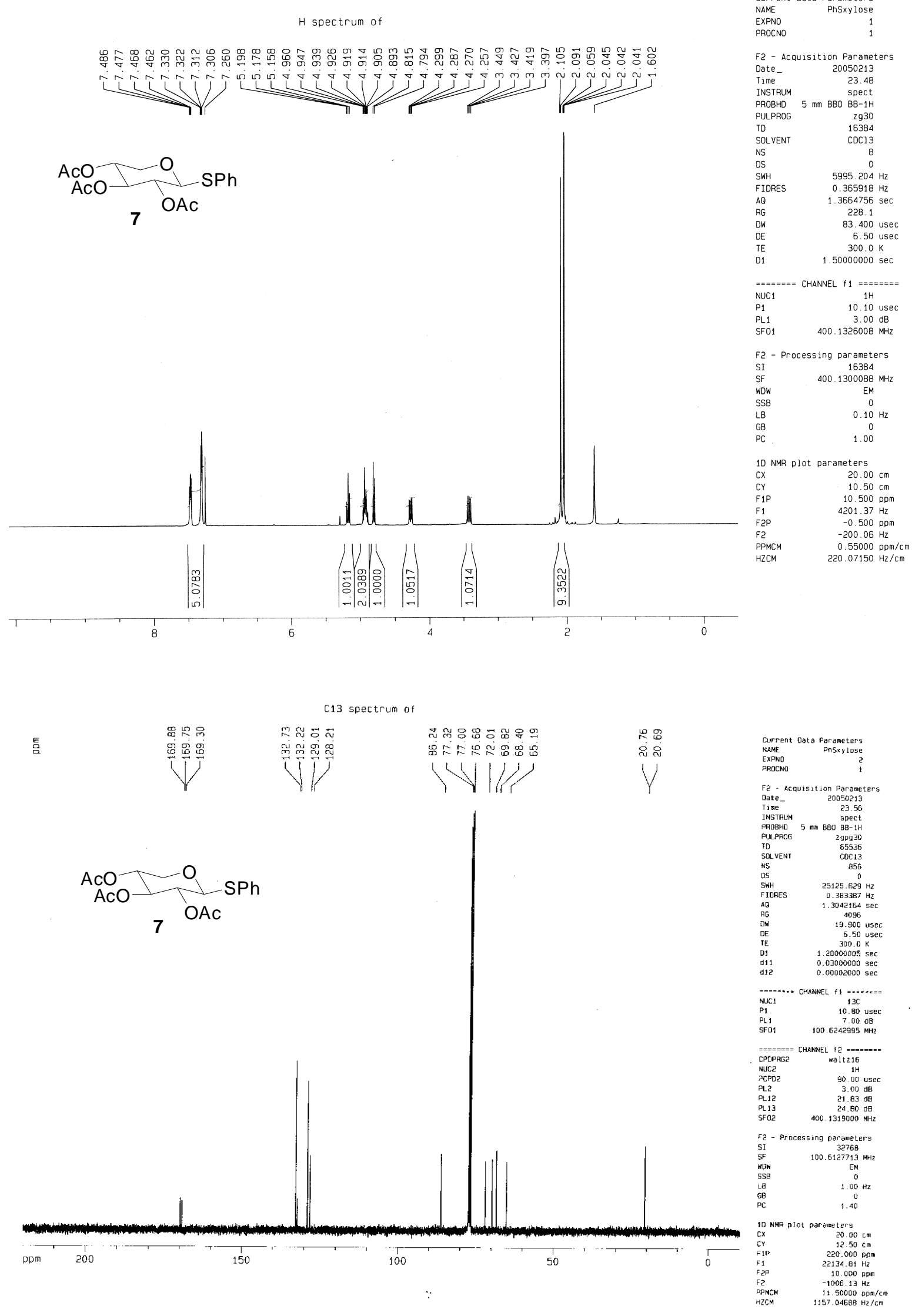


$H$ spectrum of

言

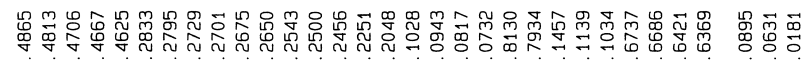

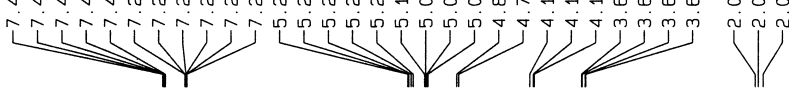<smiles>CC(=O)OC1CC(OC(C)=O)C(O)(c2ccccc2)C1OC(C)=O</smiles>

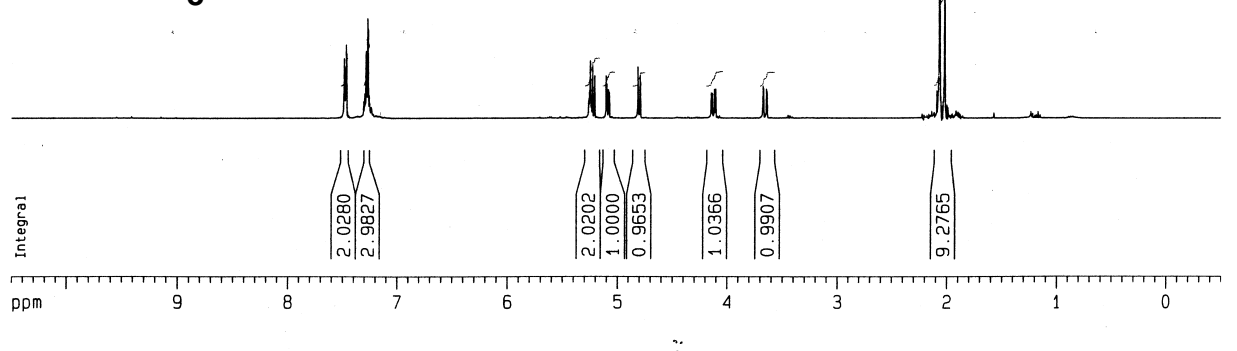

C13 spectrum of

镸
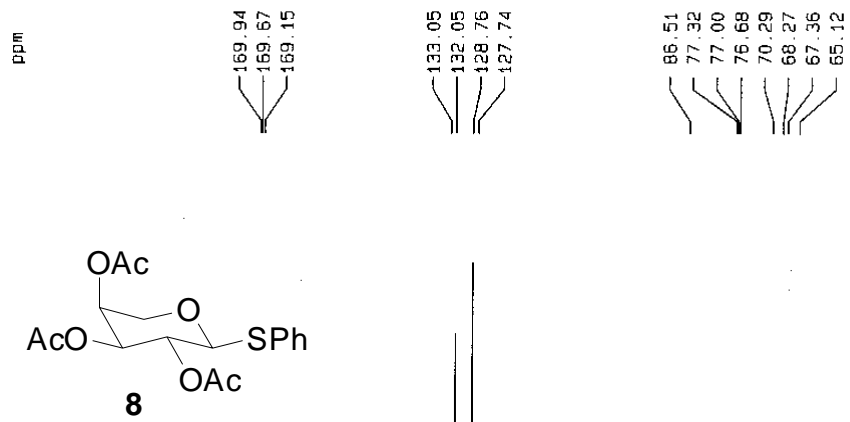

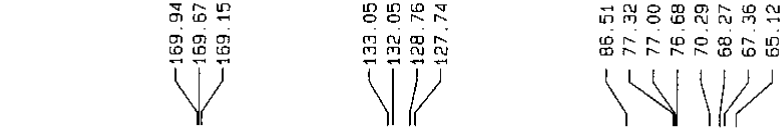

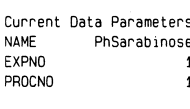

F2 - Acquisition Parameters

Date $\quad 20040427$

$\begin{array}{ll}\text { Time } & 22.36 \\ \text { INSTRUM } & \text { spect }\end{array}$ PROBHD $5 \mathrm{~mm}$ BBO BB-1H PULPROG $\quad 2930$ $\begin{array}{ll}\text { TD } & 16384 \\ \text { SOLVENT } & \text { CDC13 }\end{array}$ $\begin{array}{lr}\text { NS } & 1 \\ \text { DS } & 0\end{array}$ SWH $\quad 5995.204 \mathrm{~Hz}$ $\begin{array}{lc}\text { FIDRES } & 0.365918 \mathrm{~Hz} \\ \text { AQ } & 1.3664756 \mathrm{sec}\end{array}$

$\begin{array}{lr}\text { RG } & 22.6 \\ \text { DW } & 83.400 \mathrm{usec} \\ \text { DE } & 6.50 \mathrm{usec}\end{array}$

$50000000 \mathrm{sec}$

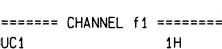

$\begin{array}{lc}\text { NuC1 } & 1 \mathrm{H} \\ \mathrm{P}_{1} & 10.10 \mathrm{usec} \\ \mathrm{P} & 3.00 \mathrm{~dB}\end{array}$

$\begin{array}{lr}\text { PL1 } & 3.00 \mathrm{~dB} \\ \text { SF01 } & 400.1326008 \mathrm{MHz}\end{array}$

F2 - Processing parameters

$\begin{array}{lr}\text { SI } & 16384 \\ \text { SF } & 400.1300050 \mathrm{MHz}\end{array}$

$\begin{array}{lr}\text { SF } & 400.1300050 \\ \text { WOW } & \text { EM } \\ \text { SSB } & 0\end{array}$

$\begin{array}{lc}S S B & 0 \\ L B & 0.10 \mathrm{~Hz} \\ \text { GB } & 0\end{array}$

$\begin{array}{ll}\text { GB } & 1.00\end{array}$

10 NMR plot parameters

$\begin{array}{ll}\text { CX } & 20.00 \mathrm{~cm} \\ \mathrm{cr} & 10.00 \mathrm{~cm}\end{array}$

$\begin{array}{ll}10.000 \mathrm{ppm} \\ \text { F1P } & 10.503 .37 \mathrm{~Hz}\end{array}$

\begin{tabular}{ll}
$-0.500 \mathrm{ppm}$ \\
\hline
\end{tabular}

$-200.07 \mathrm{~Hz}$

PPMCM $\quad 0.55000 \mathrm{ppm} / \mathrm{cm}$ $0.55000 \mathrm{ppm} / \mathrm{cm}$
$220.07152 \mathrm{~Hz} / \mathrm{cm}$
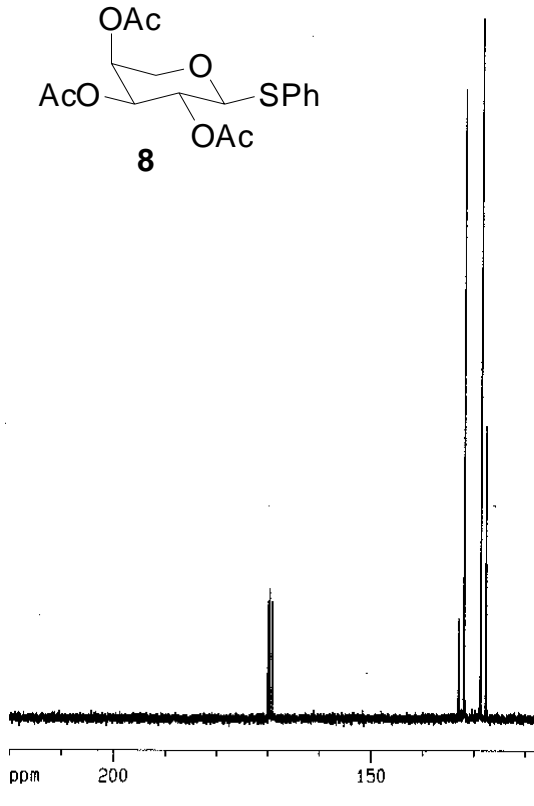

150

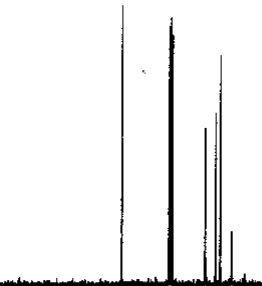

100
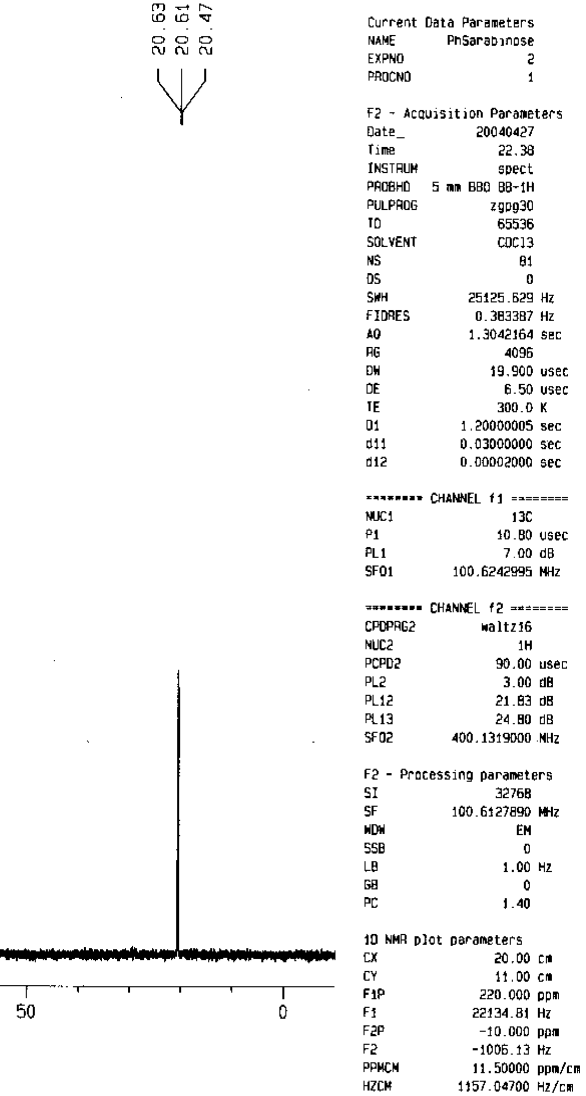

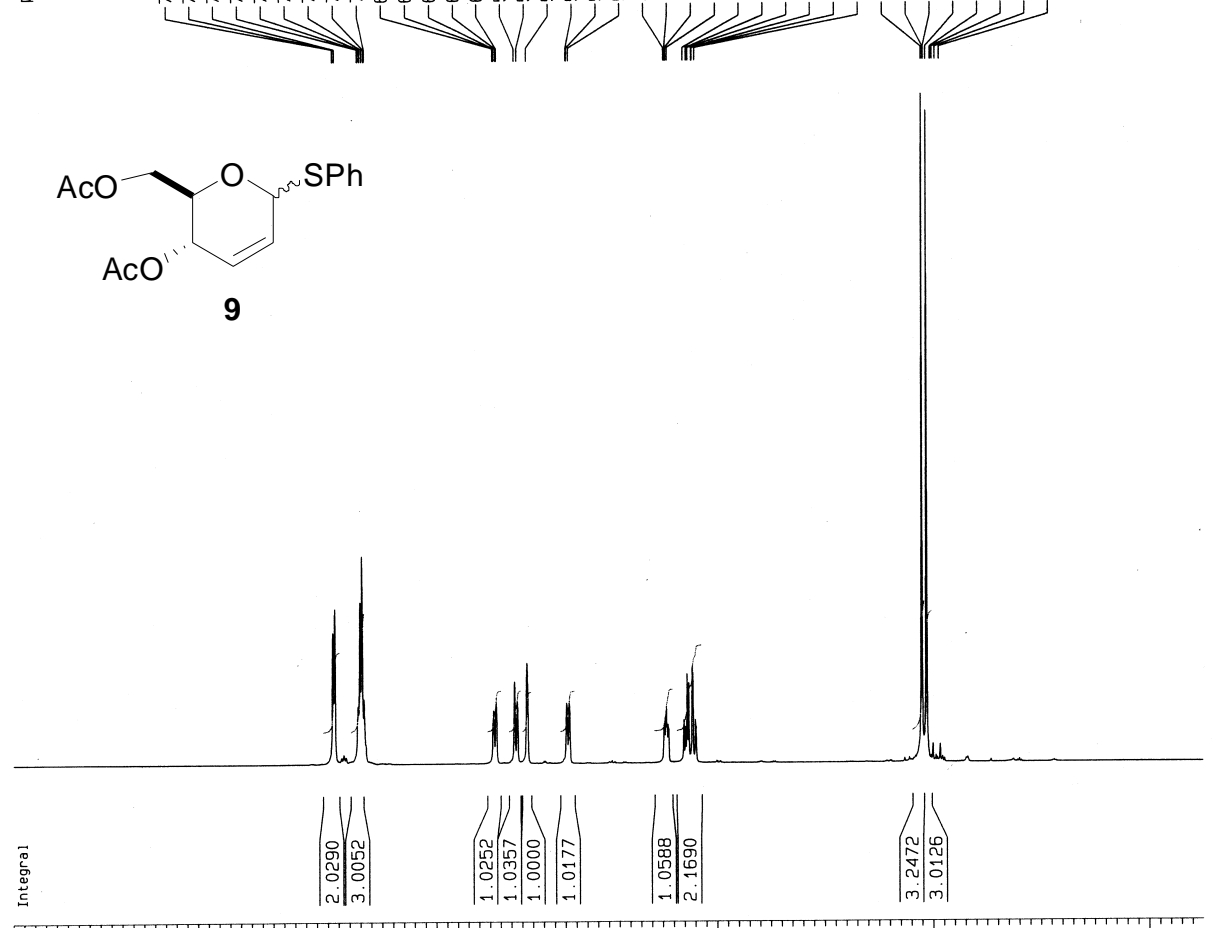

F2 - Acquisition Parameter

Date_ 2004112 ?

$\begin{array}{lr}\text { Date } & 20041122 \\ \text { Time } & 12.06\end{array}$

INSTRUM spect

PUOBHO $5 \mathrm{~mm}$ BBO BB-1H

$\begin{array}{lr}\text { PULPROG } & 2930 \\ \text { TD } & 16384 \\ \text { SOLVENT } & \text { COC13 }\end{array}$

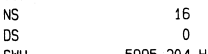

$\begin{array}{ll}\text { SWH } & 5995.204 \mathrm{~Hz} \\ \text { FIDRES } & 0.365918 \mathrm{~Hz}\end{array}$

AQ $\quad 1.3664756 \mathrm{sec}$

$\begin{array}{lr}\text { RG } & 71.8 \\ \text { DW } & 83.400 \text { usec }\end{array}$

TE $\quad 300.0 \mathrm{~K}$

$1.50000000 \mathrm{sec}$

$======$ CHANNEL $f 1=======$
NUC1 $1 \mathrm{H}$
$10.10 \mathrm{usec}$

$\begin{array}{lr}\text { PL1 } & 3.00 \mathrm{~dB} \\ \text { SF01 } & 400.1326008 \mathrm{MHz}\end{array}$

F2 - Processing parameters

SI 16384

$\begin{array}{cc}\text { WOW } & 400.1300088 \mathrm{MM} \\ \text { WSB } & \text { EM } \\ \text { SSB } & 0\end{array}$

$\begin{array}{ll}\text { LB } & 0.10 \mathrm{H} \\ G B & 0 \\ \text { GC } & 1.00\end{array}$

10 NMR plot parameters

10 NMR plot parameters
$\mathrm{CX}$
$20.00 \mathrm{~cm}$

F1P $\quad 10.500 \mathrm{ppm}$

F2P $\quad-0.500 \mathrm{pDm}$

$-200.06 \mathrm{~Hz}$

$\begin{array}{lr}\text { HPMCM } & 0.55000 \mathrm{pDm} / \mathrm{cm} \\ \mathrm{HZCM} & 220.07150 \mathrm{~Hz} / \mathrm{cm}\end{array}$

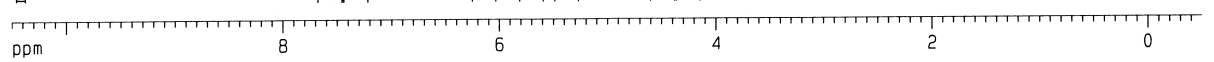

C13 spectrum of

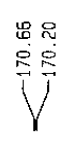

ミ三罚罢思

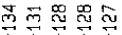

ij

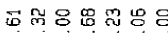

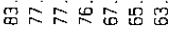

JYII

웅웅
용
$y$
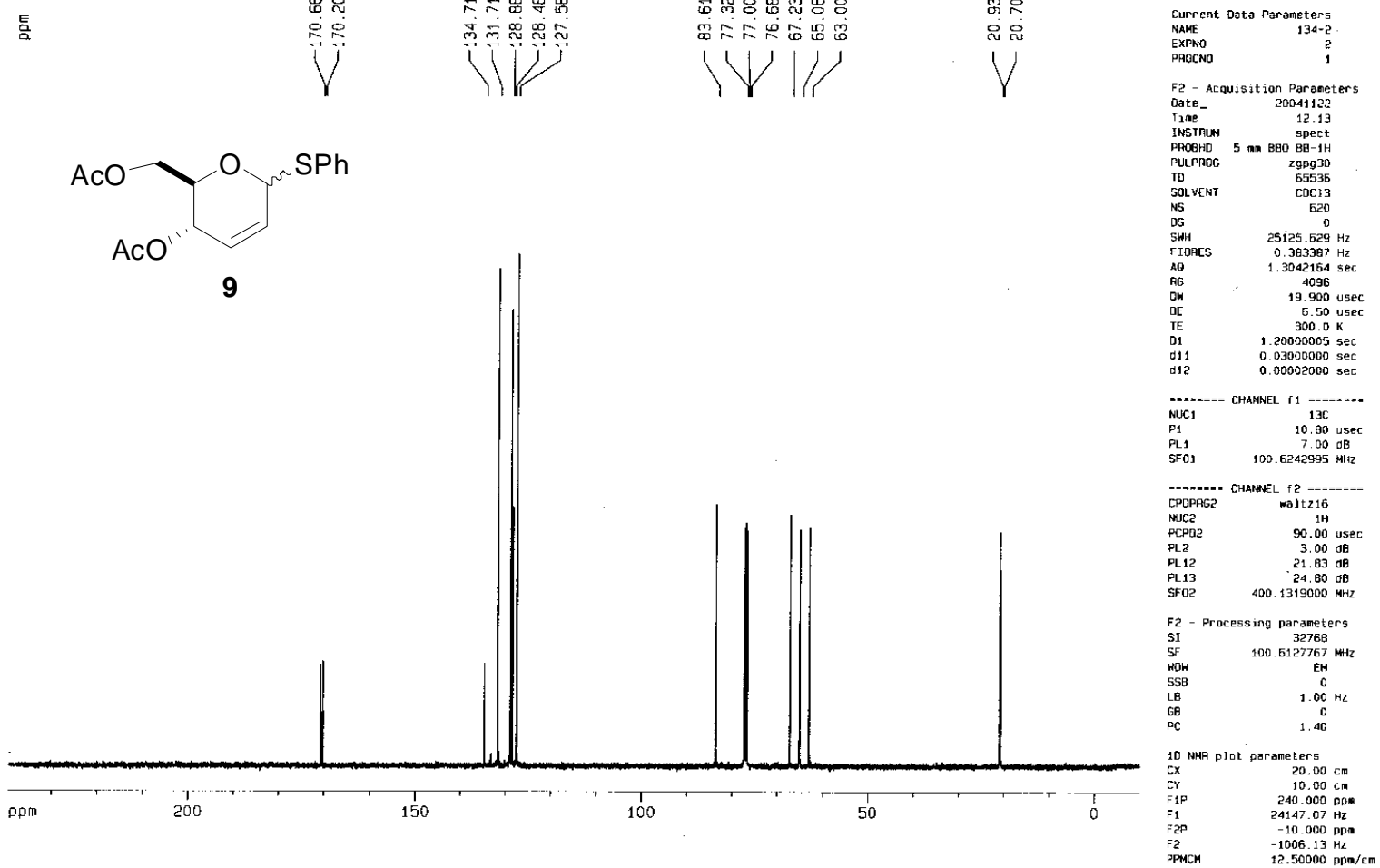
$H$ spectrum of

言
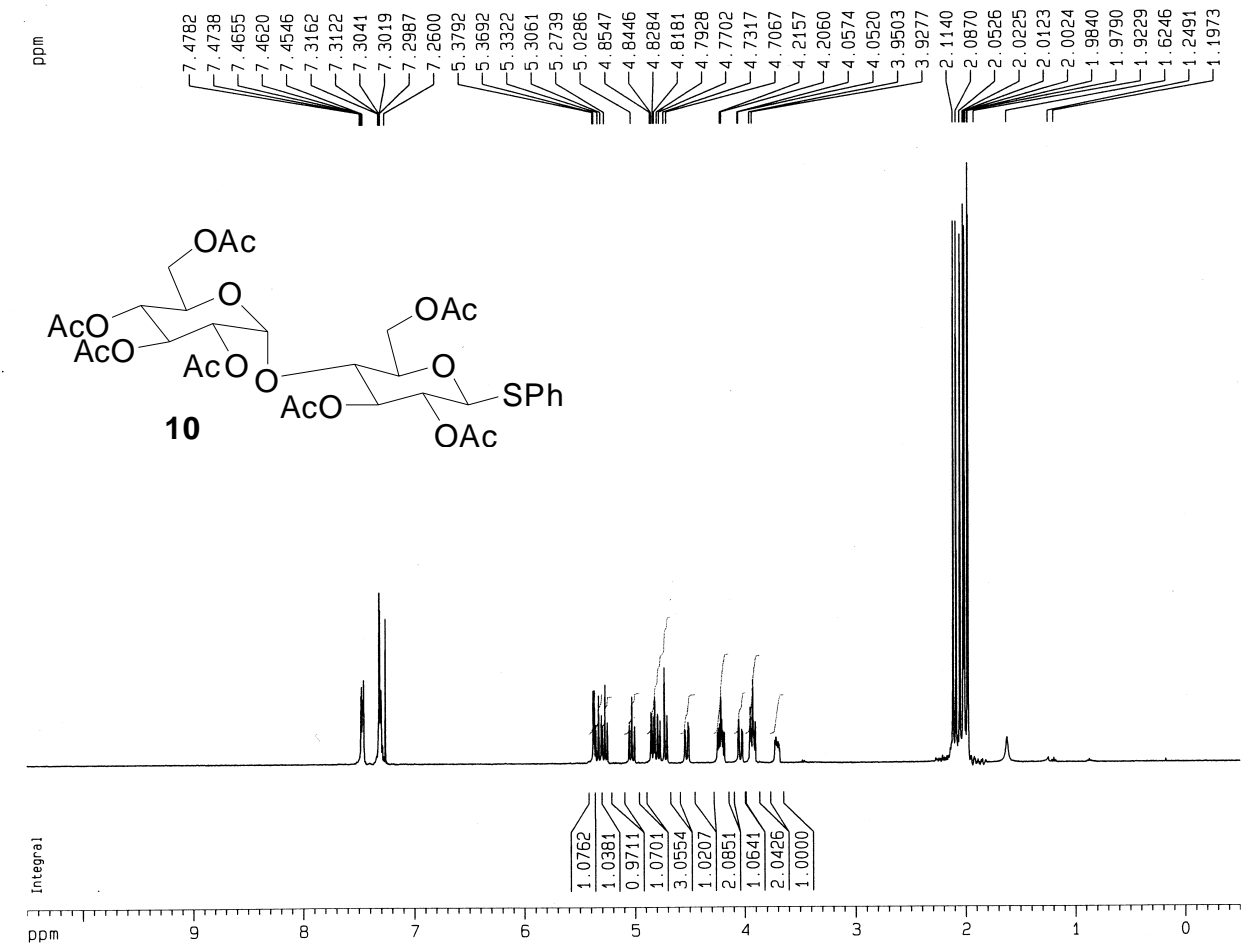

C13 spectrum of
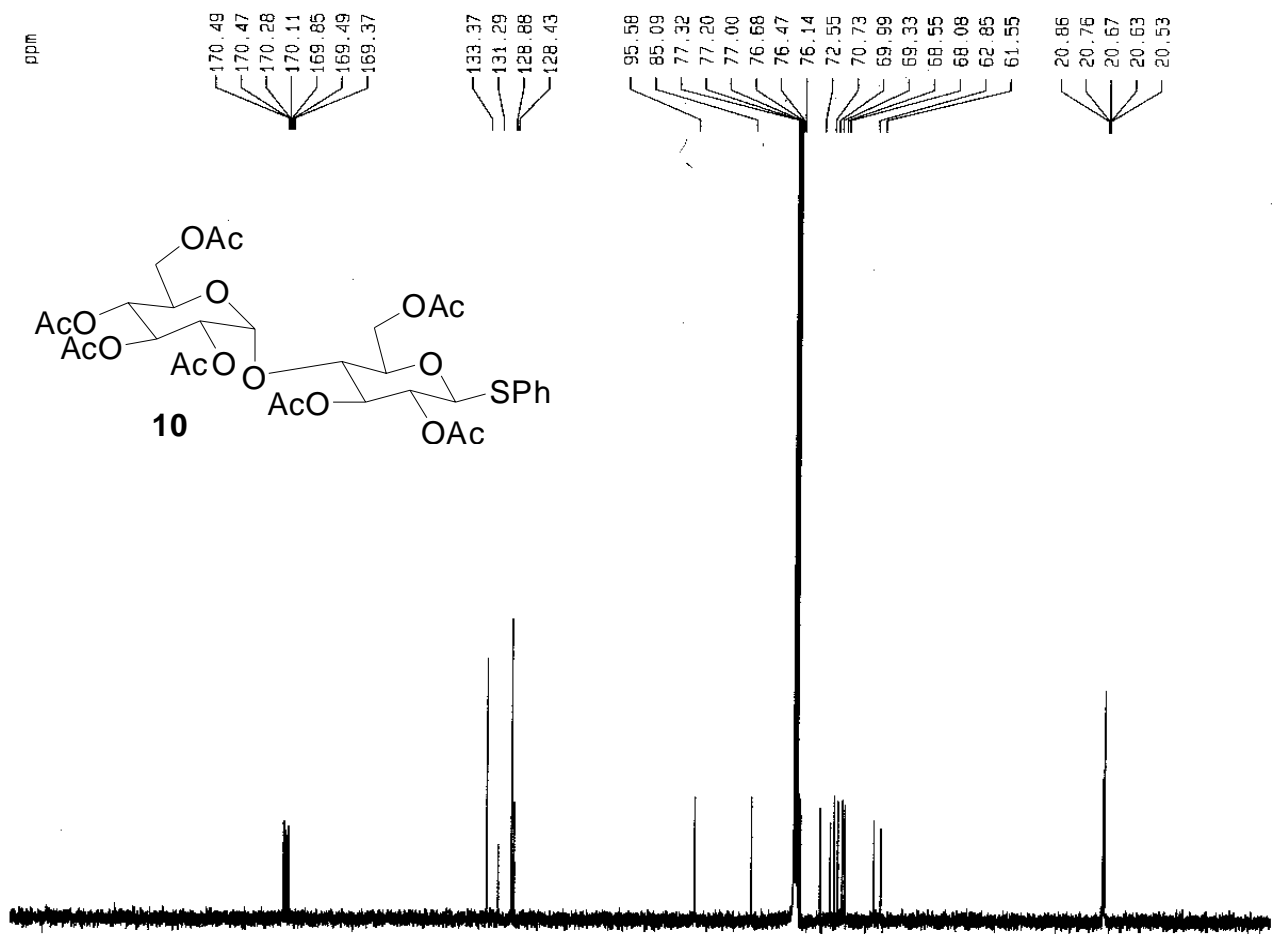

pp

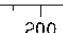

175

150

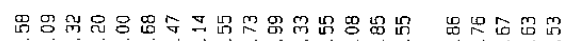

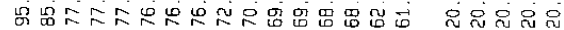

$L_{1}^{L L} \mid$

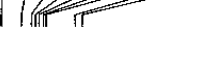

75

25

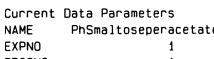

F2 - Acquisition Parameters

20040420
Time

spect
INSTRUM
PROBHD $5 \mathrm{~mm}$ BBO BB-1H

2930
PULPROG

$\begin{array}{lc}\text { NS } & \text { COC13 } \\ \text { NS } & 1 \\ \text { OS } & 0 \\ \text { SWH } & 5995.204 \mathrm{~Hz}\end{array}$

$\begin{array}{ll}\text { HDPES } & 0.365918 \mathrm{~Hz} \\ 1.3654756 \mathrm{se} & \end{array}$

$\begin{array}{lr}A Q & 1.3664756 \mathrm{sec} \\ \mathrm{AG} & 113.7 \\ \mathrm{PG} & 83.400 \mathrm{usec}\end{array}$

6.50 use
TE
$300.0 \mathrm{~K}$

....... CHANNEL $f 1=====$

$\begin{array}{ll}\text { NuC1 } & 1 \mathrm{H} \\ \text { P1 } & 14.30 \mathrm{usec} \\ \text { PL1 } & -5.00 \mathrm{~dB}\end{array}$

SF01 400.1326008 MH

F2 - Processing parameters

$\begin{array}{lc}\text { SI } & 16384 \\ \text { SF } & 400.1300095 \mathrm{MHz} \\ \text { KOW } & \mathrm{EM} \\ \text { SSB } & 0 \\ \text { LB } & 0.10 \mathrm{~Hz} \\ \text { GB } & 0 \\ \text { PC } & 1.00\end{array}$

10 NMR plot parameters

$\begin{array}{lr}10 \text { NMA plot parameters } \\ \text { CX } & 20.00 \mathrm{~cm} \\ \text { CY } & 10.50 \mathrm{~cm} \\ \text { F1P } & 10.500 \mathrm{ppm} \\ \text { F1 } & 4201.37 \mathrm{~Hz} \\ \text { F2P } & -0.500 \mathrm{ppm} \\ \text { F2 } & -200.07 \mathrm{~Hz} \\ \text { PPMCM } & 0.55000 \mathrm{pom} / \mathrm{cm} \\ \text { HZCM } & 220.07152 \mathrm{~Hz} / \mathrm{cm}\end{array}$

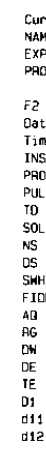

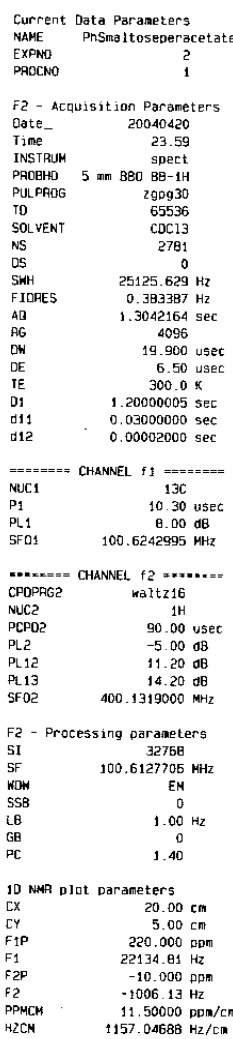




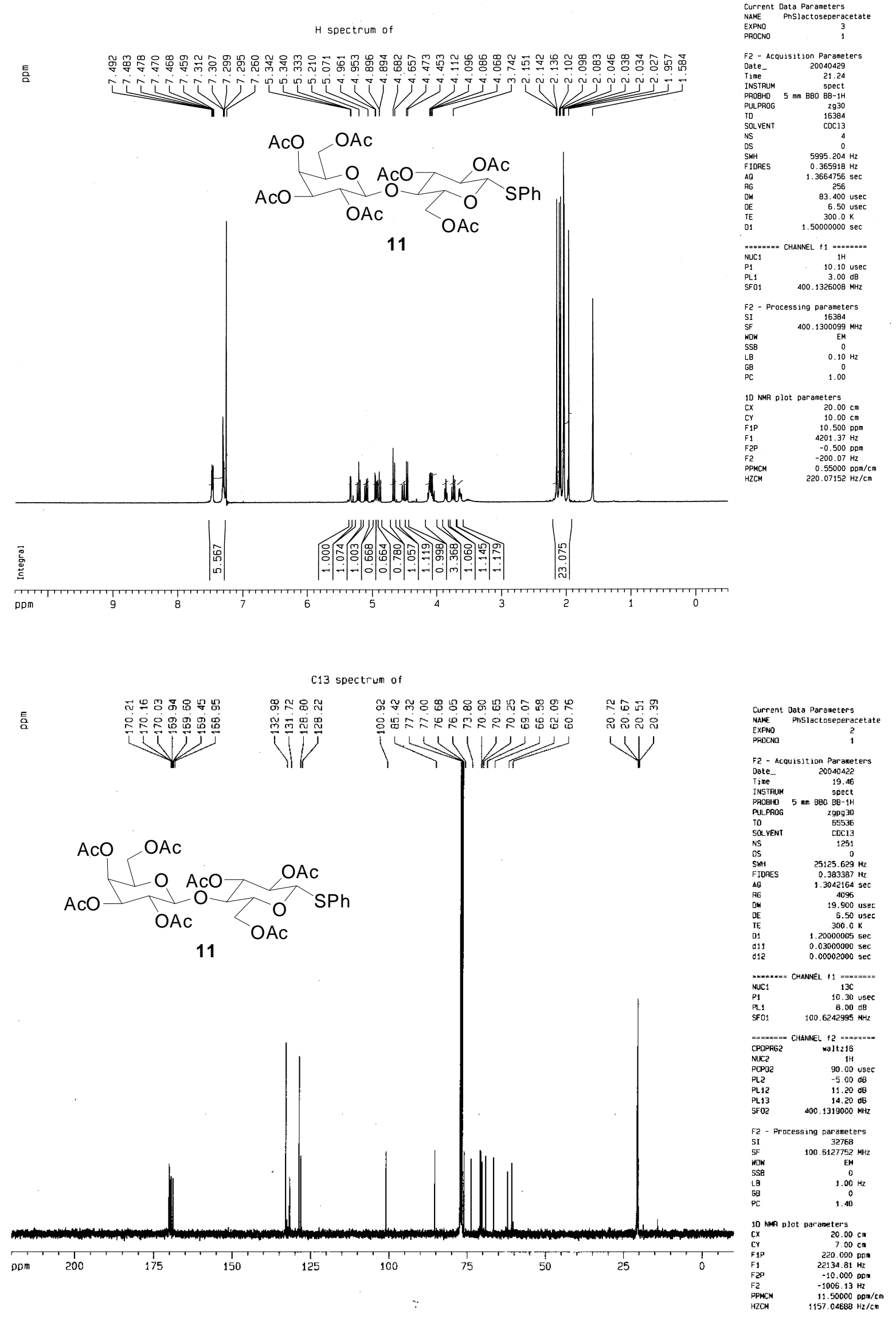


言

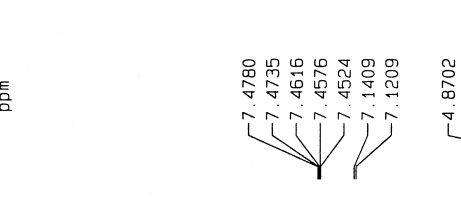

$H$ spectrum of
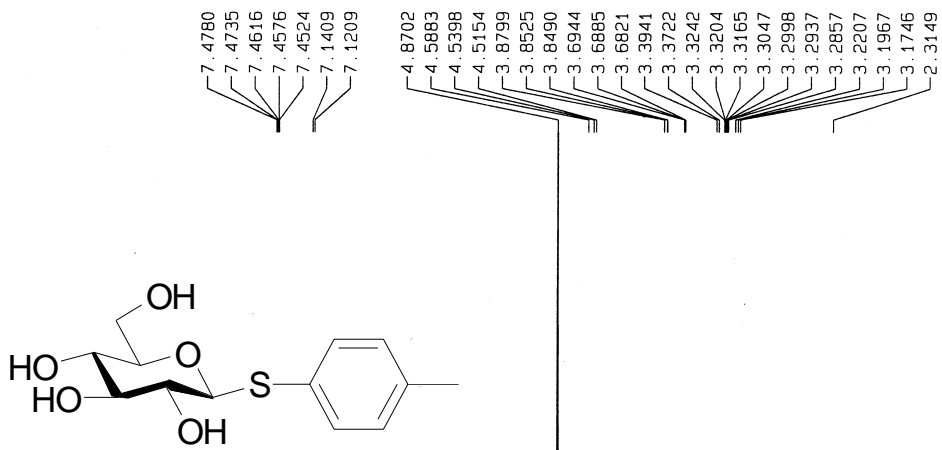

12'

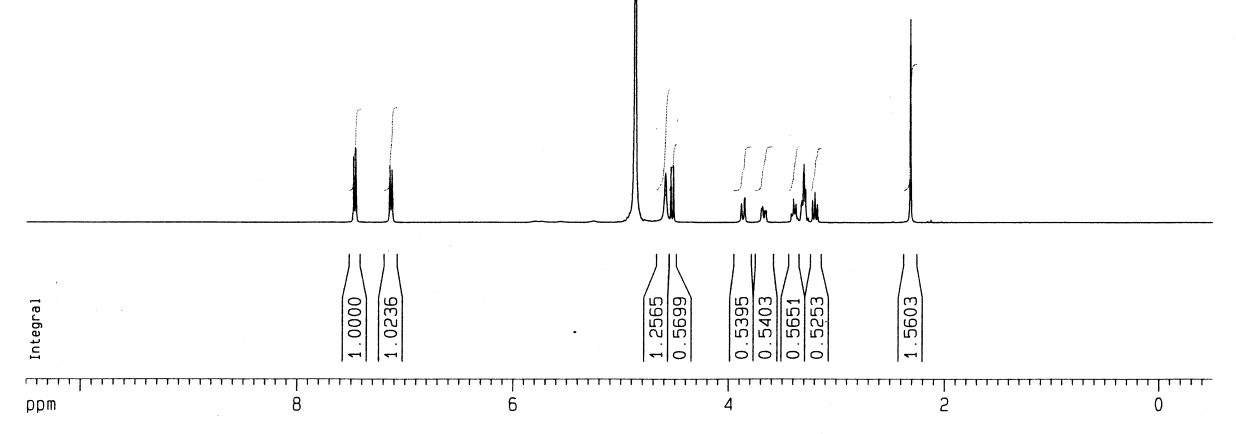

C13 spectrum of
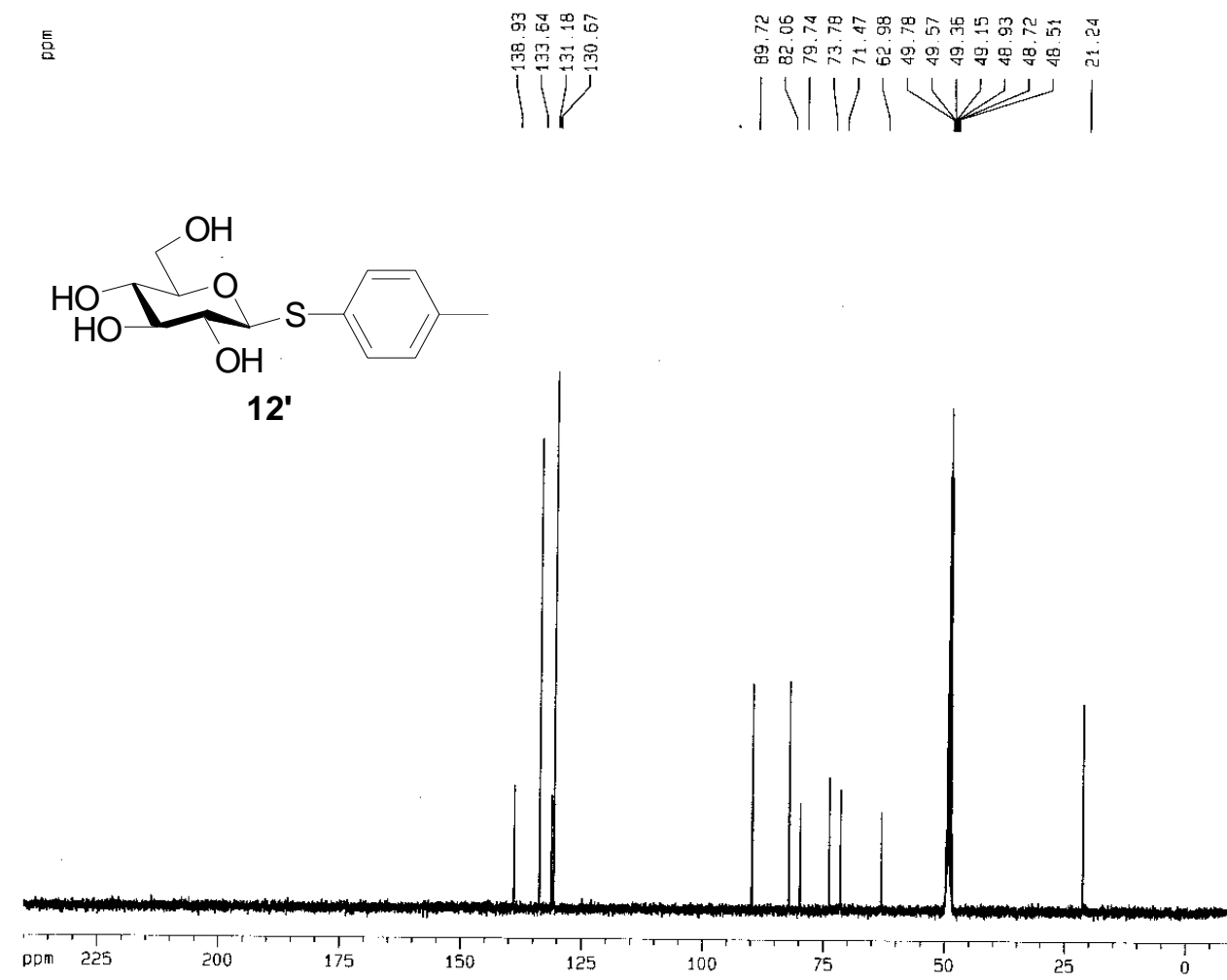

Current Data Parameters
NaME STolglucose

$\begin{array}{lr}\text { EXPNO } & \text { STOLIgluCOSE } \\ \text { PROCNO } & 1\end{array}$

F2 - Acquisition Parameters

Date_ 20040701

Time

INSTRUM spect

PULPROG $5 \mathrm{~mm}$ BBO BB-1

TD 16384

$\begin{array}{lr}\text { SOLVENT } & \text { MeOD } \\ \text { NS } & 8 \\ \text { DS } & 0\end{array}$

$\begin{array}{ll}\text { SWH } & 5995.204 \mathrm{~Hz} \\ \text { FIDRES } & 0.365918 \mathrm{~Hz}\end{array}$

AQ $\quad 1.3664756 \mathrm{sec}$

45.3
83.400 us

6.50 usec $1.50000000 \mathrm{sec}$

$==s=z==$ CHANNEL $f 1== \pm= \pm==$

$\begin{array}{ll}\text { NUC1 } & 1 \mathrm{H} \\ P_{1} & 10.10 \mathrm{usec}\end{array}$

$\begin{array}{lr}\text { PL1 } & 100 \mathrm{~dB} \\ \text { SFO } & 400.1325008 \mathrm{MHz}\end{array}$

F2 - Processing parameters

SI - Processing parameters 16384

$\quad 400.1300034 \mathrm{MHz}$

$\begin{array}{lc}\text { SSB } & 0 \\ L B & 0.10 \mathrm{~Hz}\end{array}$

$\begin{array}{lr}\text { GB } & 1.00\end{array}$

10 NMR plot parameters

CX

$20.00 \mathrm{~cm}$
$15.00 \mathrm{~cm}$ $10.500 \mathrm{ppm}$
$4201.37 \mathrm{~Hz}$ $-0.500 \mathrm{ppm}$

$-200.06 \mathrm{~Hz}$

$0.55000 \mathrm{ppm} / \mathrm{cm}$
$220.07150 \mathrm{~Hz} / \mathrm{cm}$ 
$H$ spectrum of

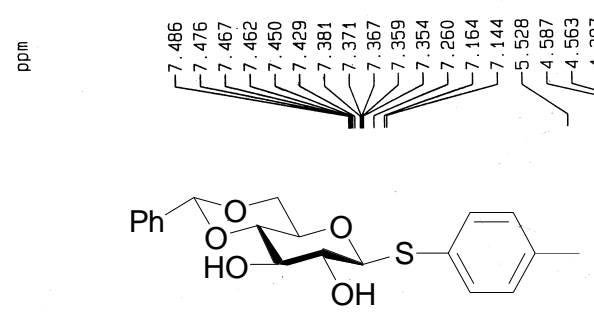

12

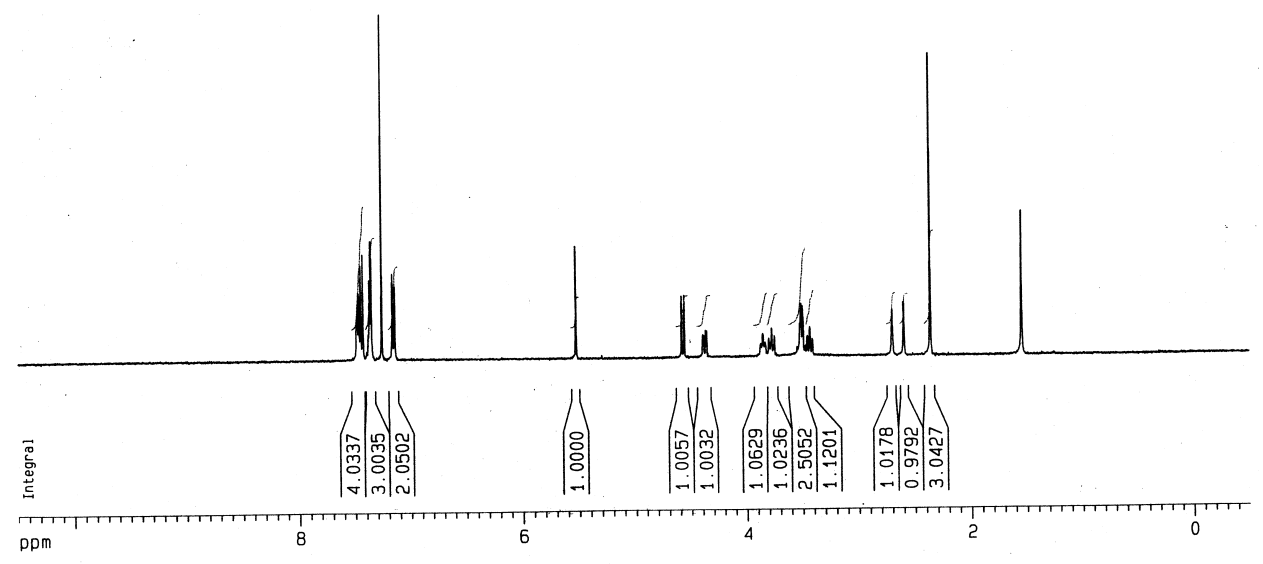

C13 spectrum of
Current Data Parameters

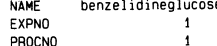

F2 - Acquisition Parameters

Date_ 20030731

Time

PROBHD $5 \mathrm{~mm}$ BBO BB-1H

$\begin{array}{lr}\text { PULPROG } & 2930 \\ & 16384\end{array}$

$\begin{array}{lr}\text { TO } & \text { COLNENT } \\ \text { NS } & \text { COC13 } \\ \text { NS } & 0\end{array}$

$\begin{array}{lc}\text { NS } & 2 \\ \text { DS } & 0 \\ & 5995.204 \\ \text { HZ }\end{array}$

$\begin{array}{ll}\text { FIDRES } & 0.365918 \mathrm{~Hz} \\ & 1.3664756 \mathrm{sec}\end{array}$

RG $\quad 406.4$

$\begin{array}{cc}\text { OW } & 83.400 \mathrm{usec} \\ \text { OE } & 6.50 \mathrm{usec} \\ \text { TE } & 300.0 \mathrm{~K}\end{array}$

$1.50000000 \mathrm{sec}$

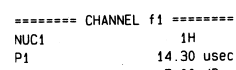

$\begin{array}{lr}\text { P1 } & 14.30 \text { usec } \\ \text { PL1 } & -5.00 \mathrm{~dB} \\ \text { SF01 } & 400.132500 \mathrm{MHz}\end{array}$

- Processing parameters

SI Processing parameters

WOW $\quad 400.1300095 \mathrm{MH}$

$\begin{array}{lc}\text { SSB } & 0 \\ \text { LB } & 0.10 . \mathrm{Hz} \\ \text { GB } & 0 \\ \text { PC } & 1.00 .\end{array}$

10 NMR plot parameters

$\begin{array}{lr}\text { CX } & 20.00 \mathrm{~cm} \\ \mathrm{CY} & 5.00 \mathrm{~cm}\end{array}$

$\begin{array}{lr}\text { F1P } & 10.500 \mathrm{ppm} \\ \text { F1 } & 4201.37 \mathrm{~Hz}\end{array}$

$\begin{array}{ll}\text { F2P } & -0.500 \mathrm{ppm} \\ \text { F2 } & -200.06 \mathrm{~Hz}\end{array}$

PPMCM $\quad 0.55000 \mathrm{ppm} / \mathrm{c}$
言

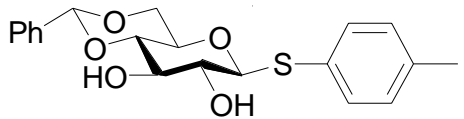

12

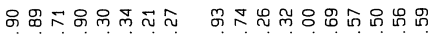

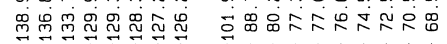

jरj

$\overrightarrow{1}$

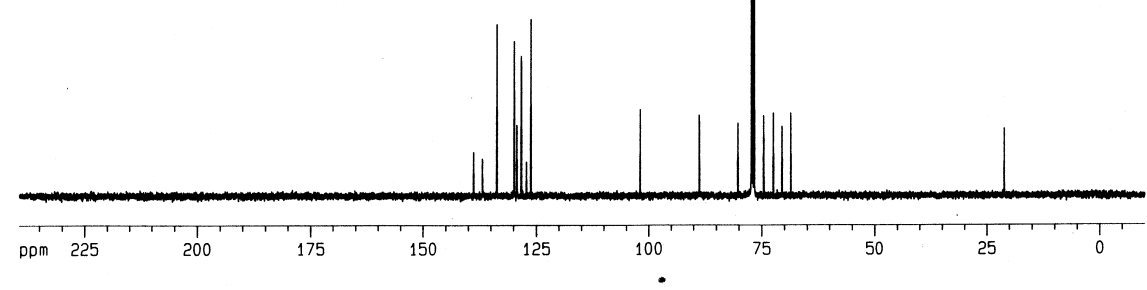

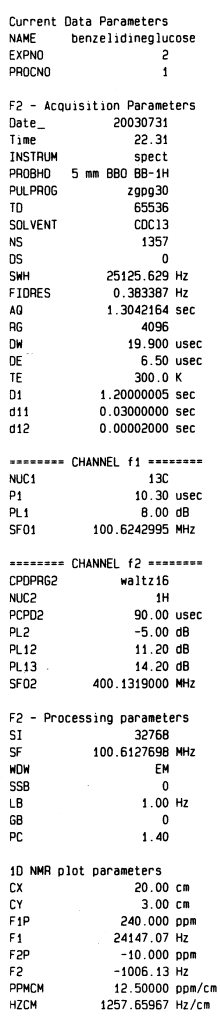



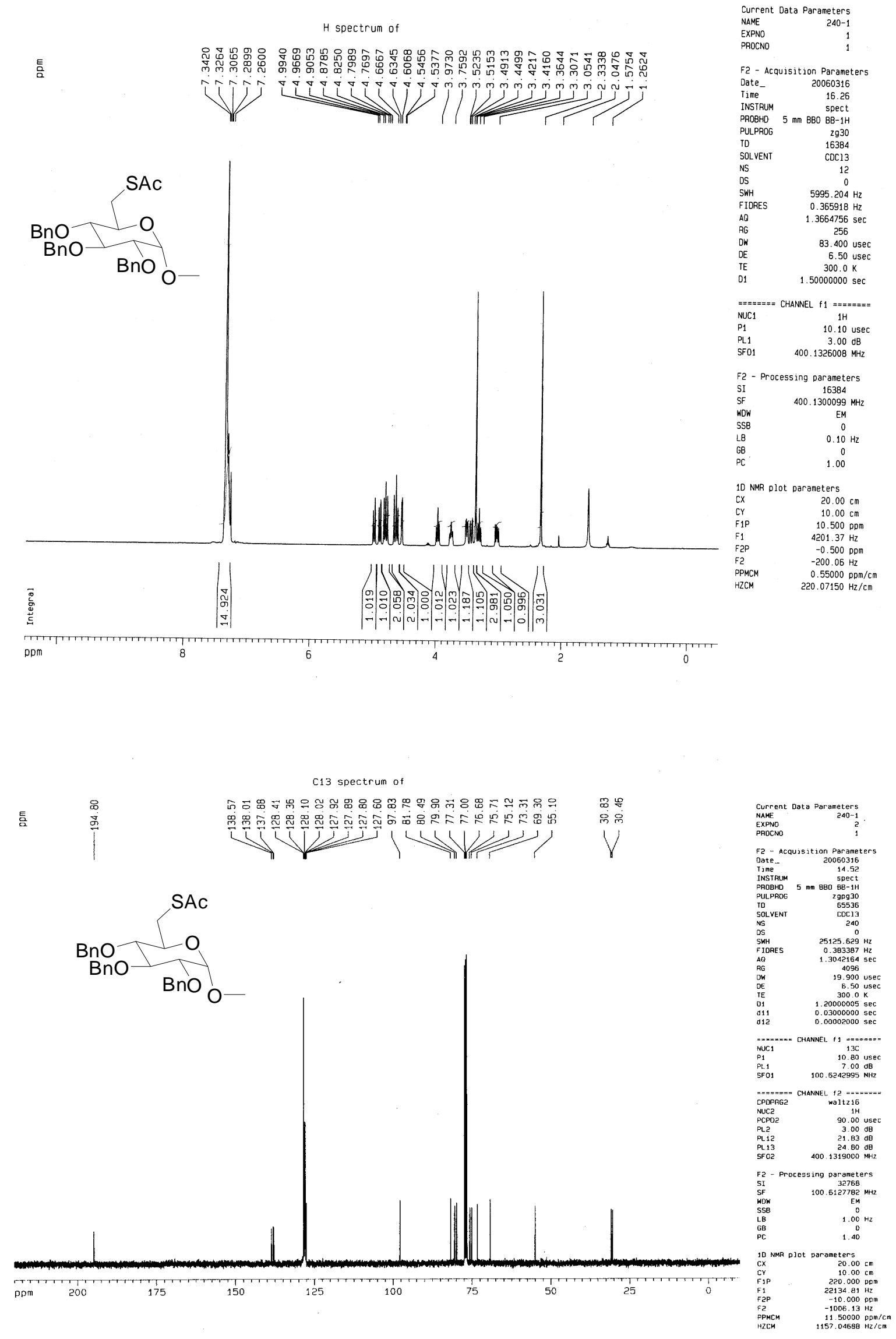
H spectrum of

言

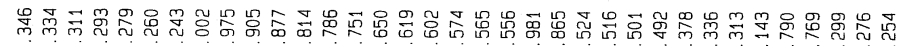

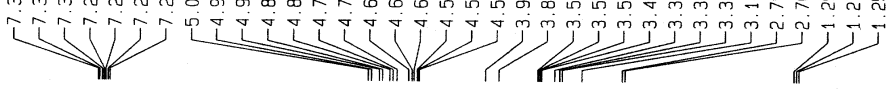

BnO

14
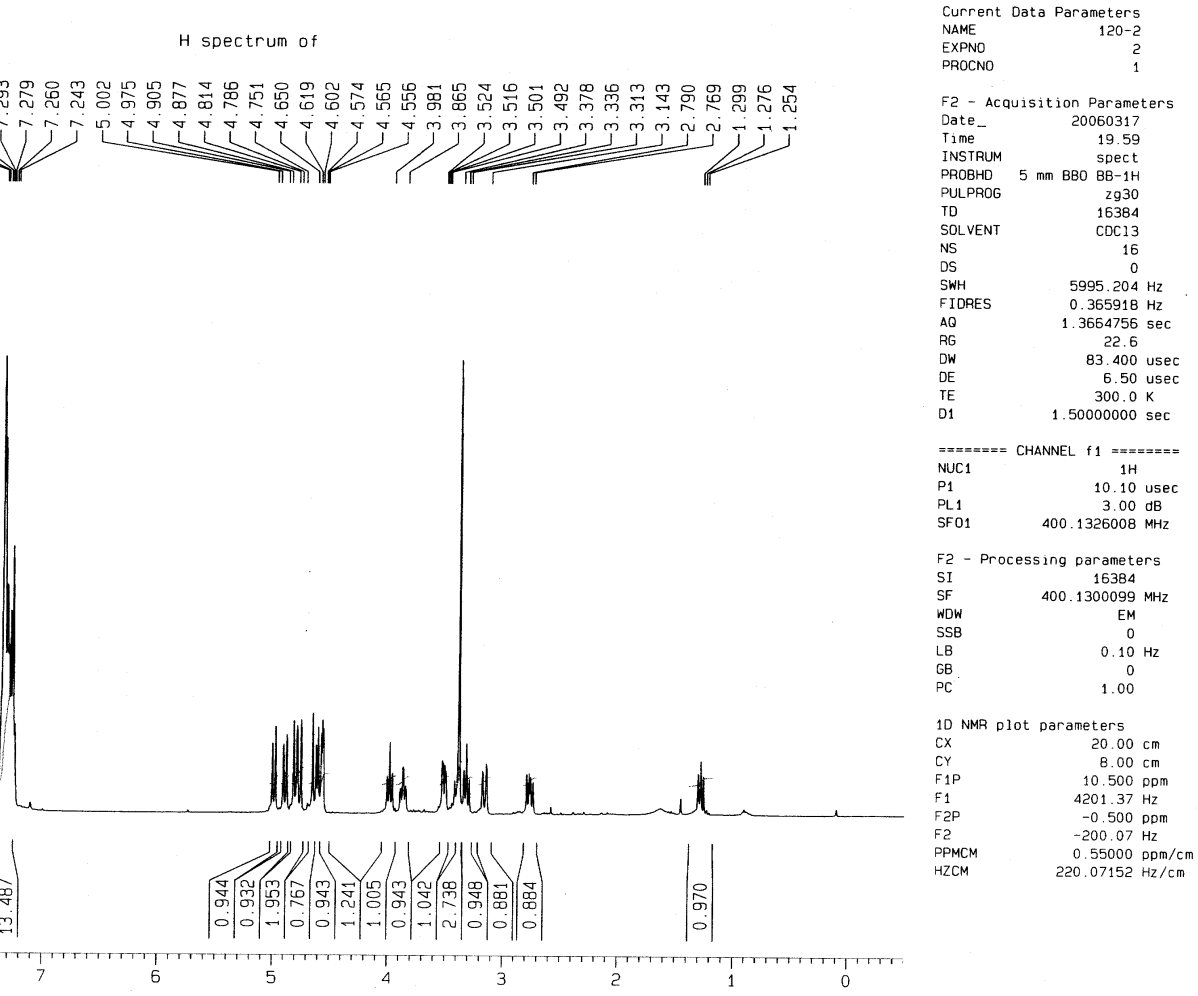

C13 spectrum of

言

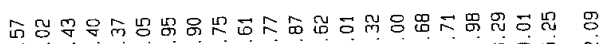

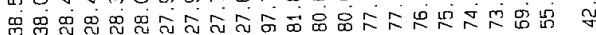

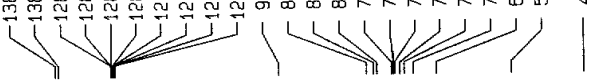<smiles>OC1C2COC(O2)C(Br)(Br)C1(Br)Br</smiles>

14
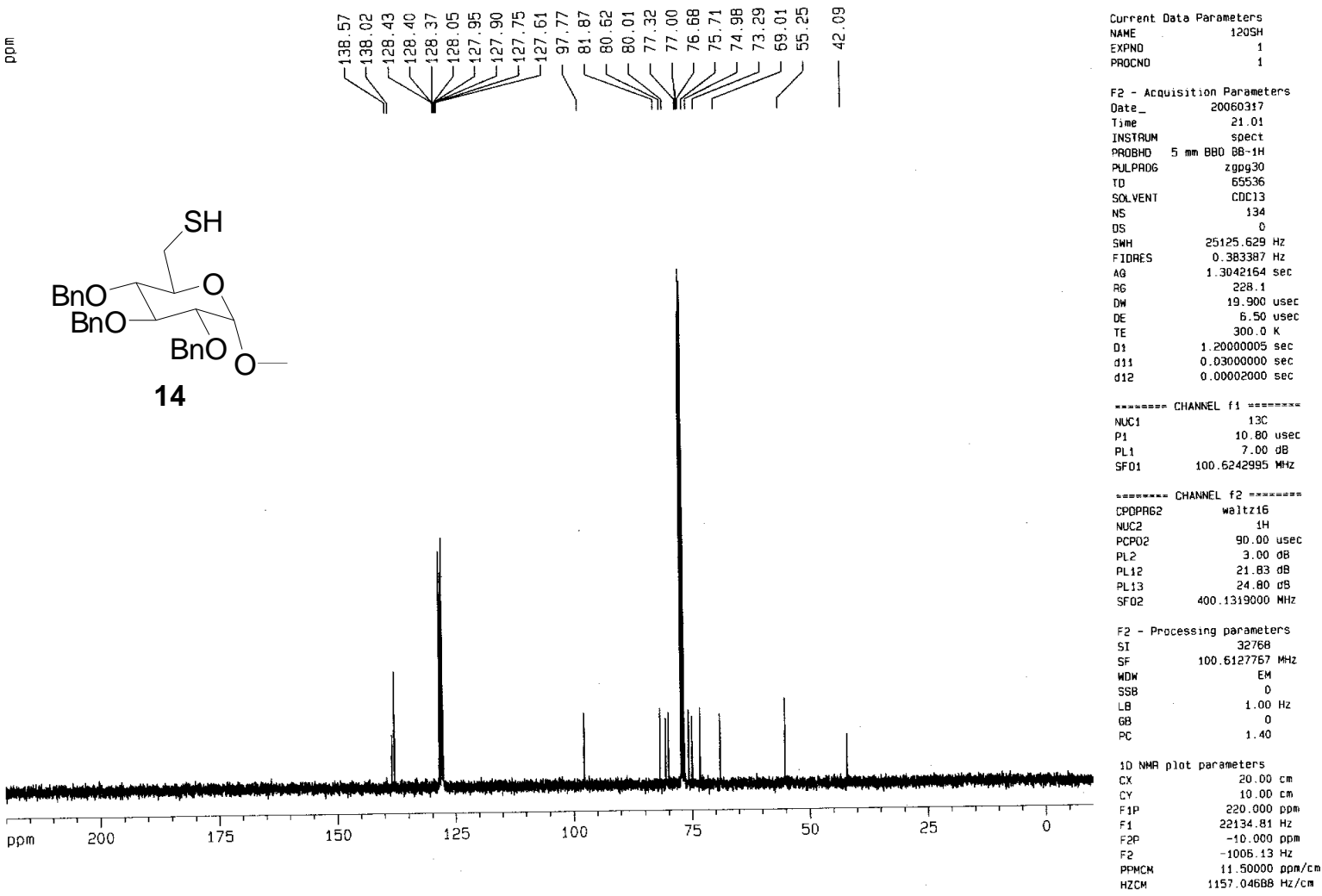


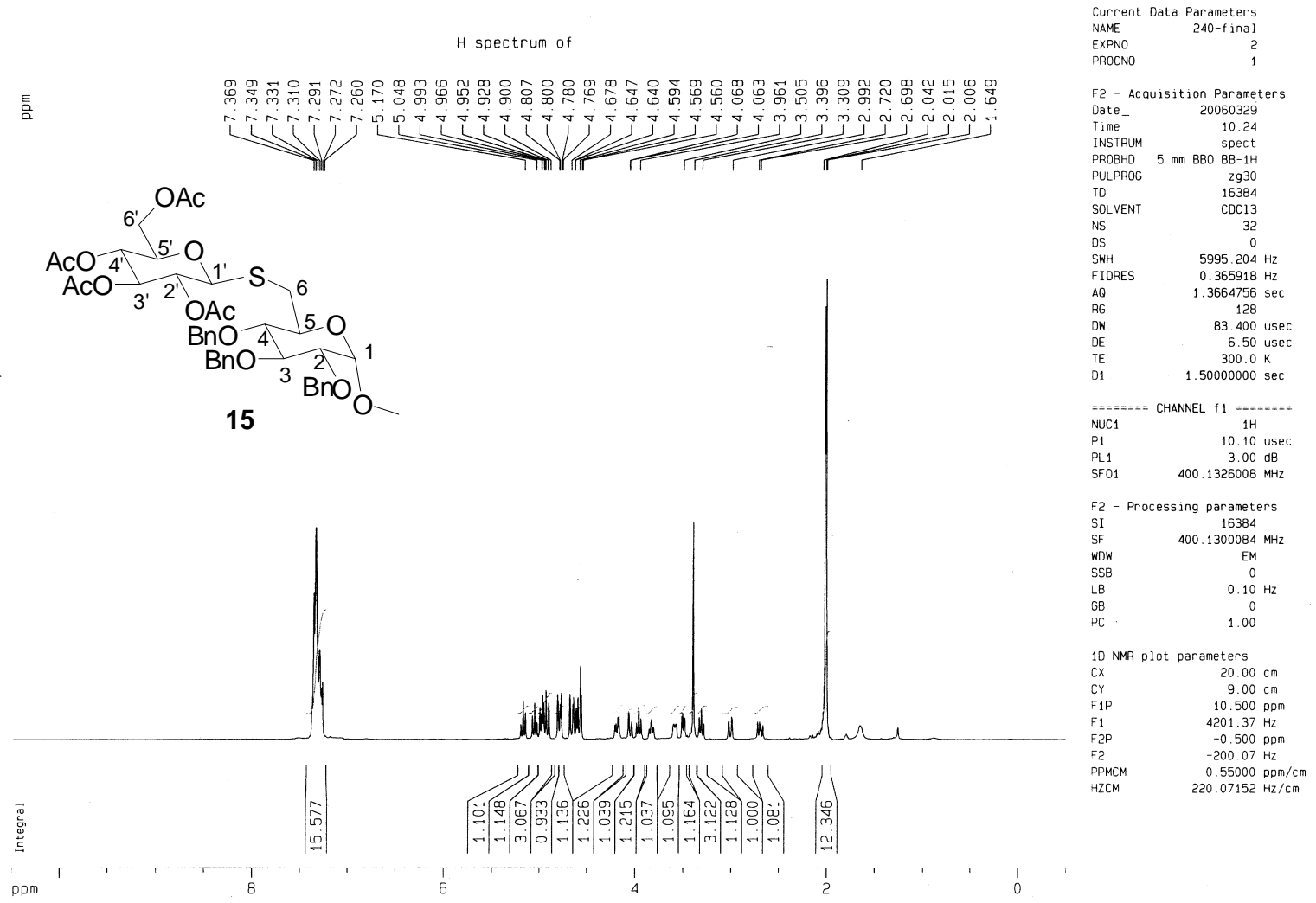

$H$ spectrum of

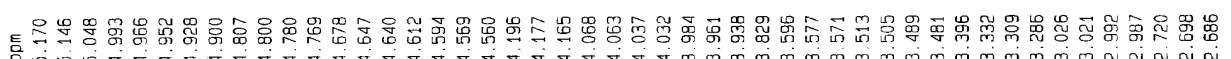
(1)

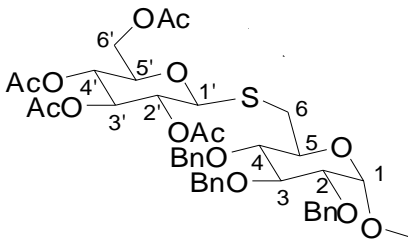
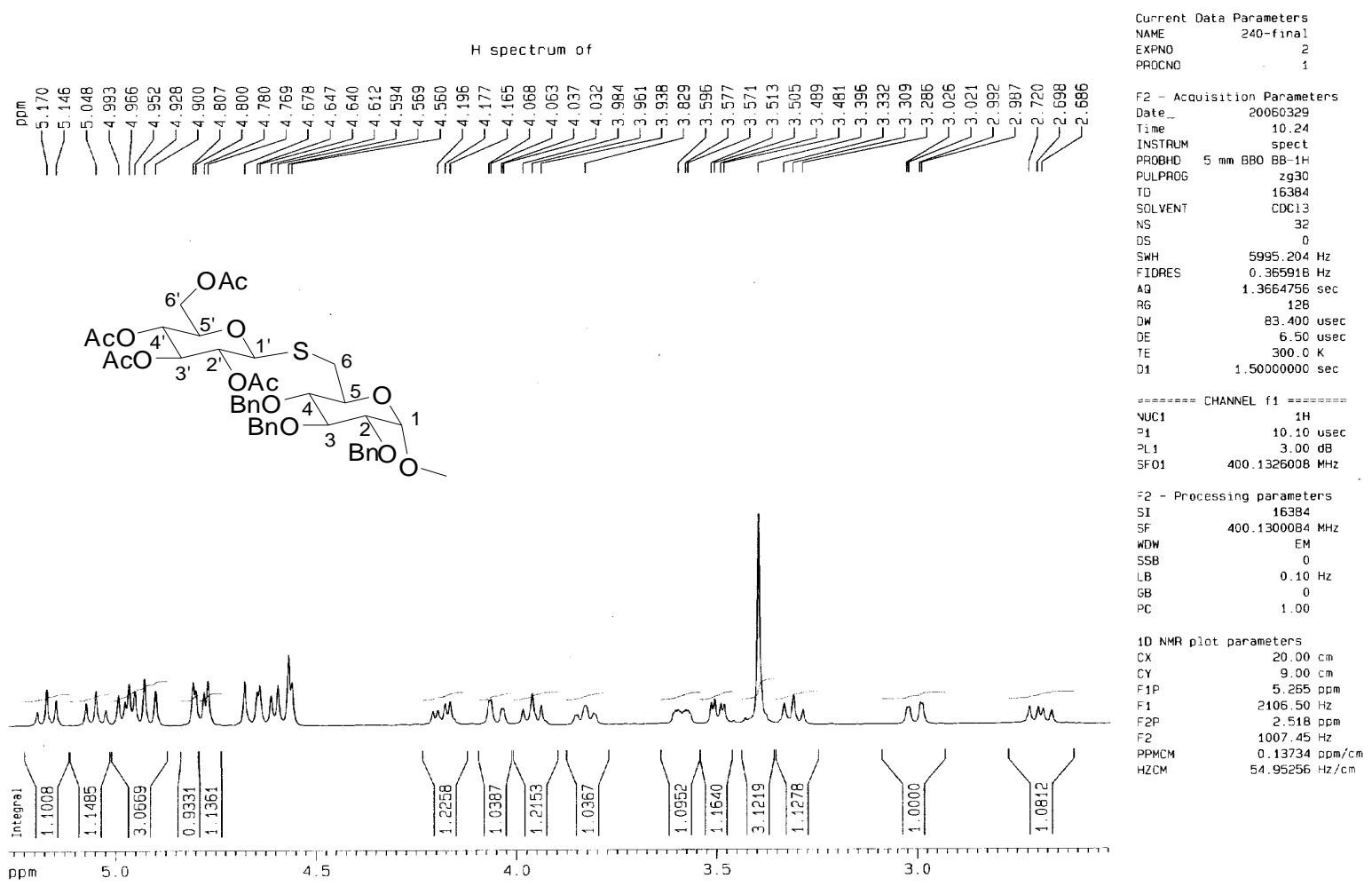


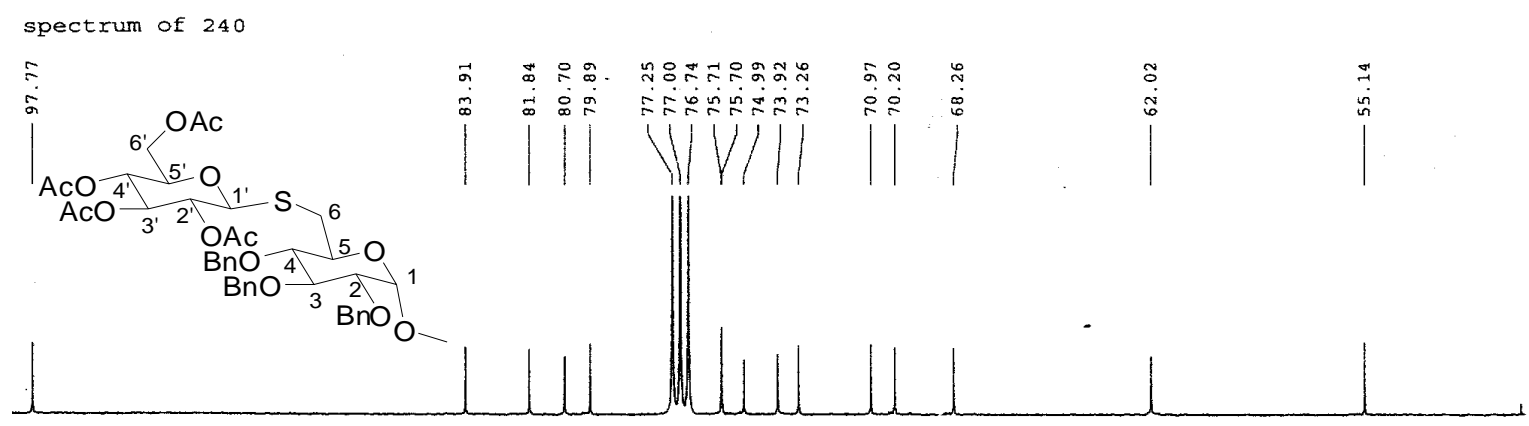

PT-135 spectrum of 240

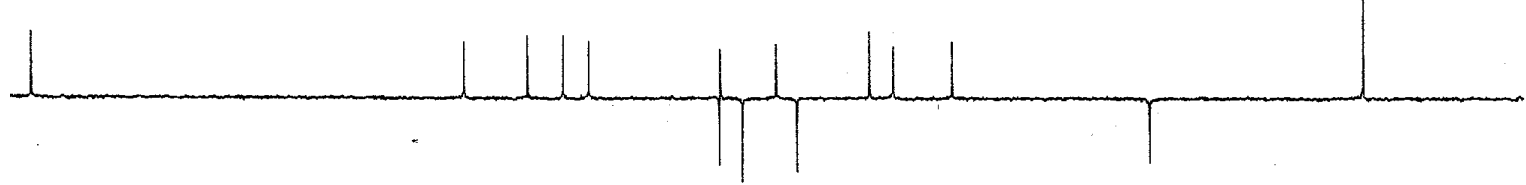

:PT-90 spectrum of 240

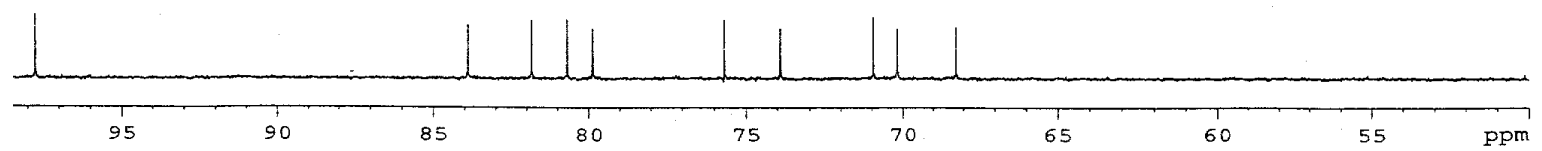

C13 spectrum of

틈

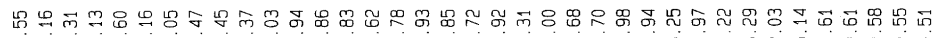

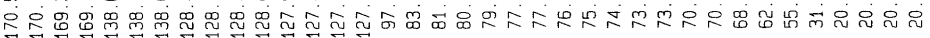
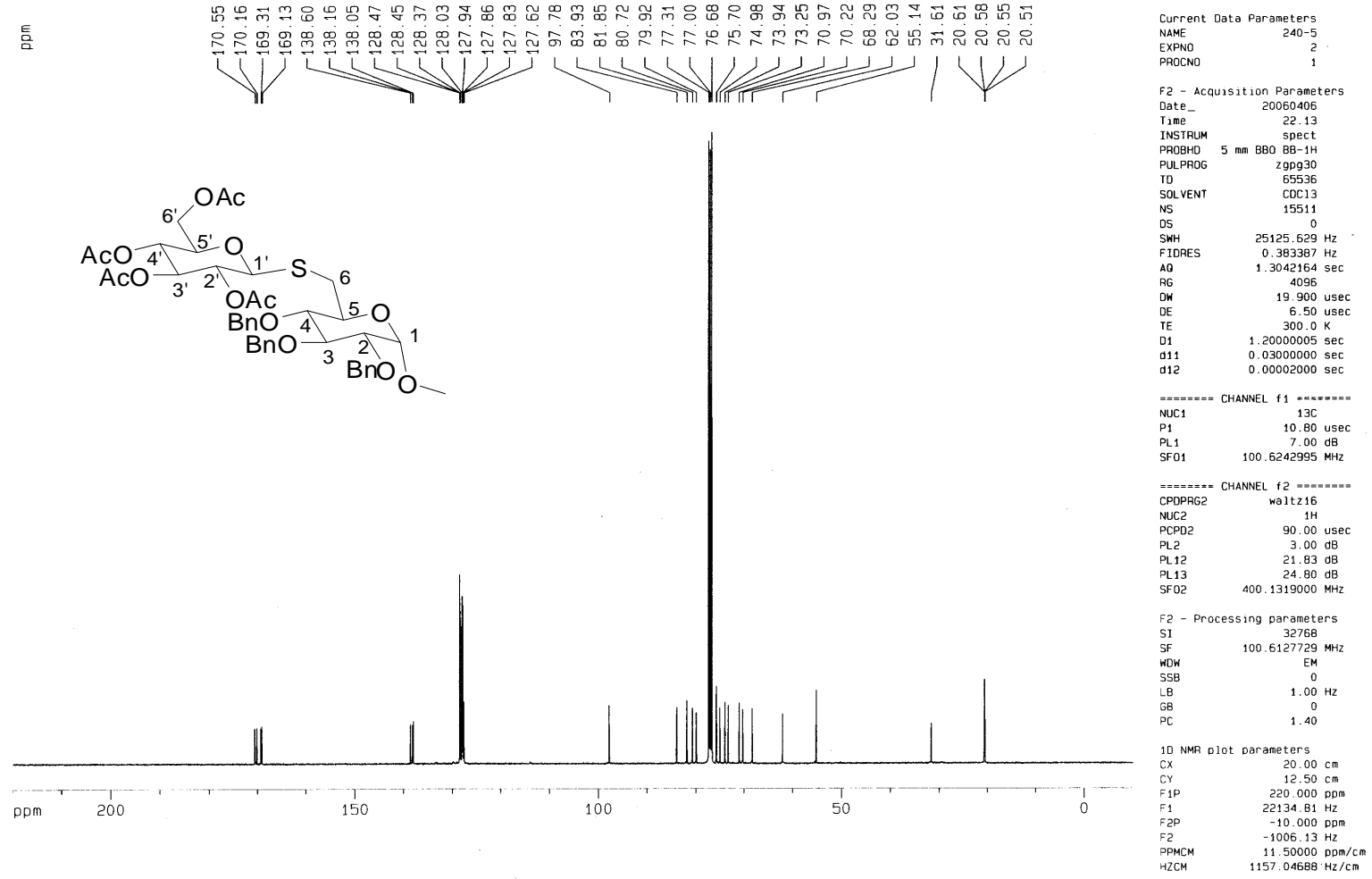

pp

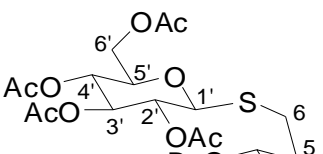

$$
\begin{aligned}
& \mathrm{BnO}^{\frac{4}{3} \mathrm{~B}^{2}}{ }^{\mathrm{BnO}}{ }^{1}
\end{aligned}
$$$$
\text { (1) }
$$ 

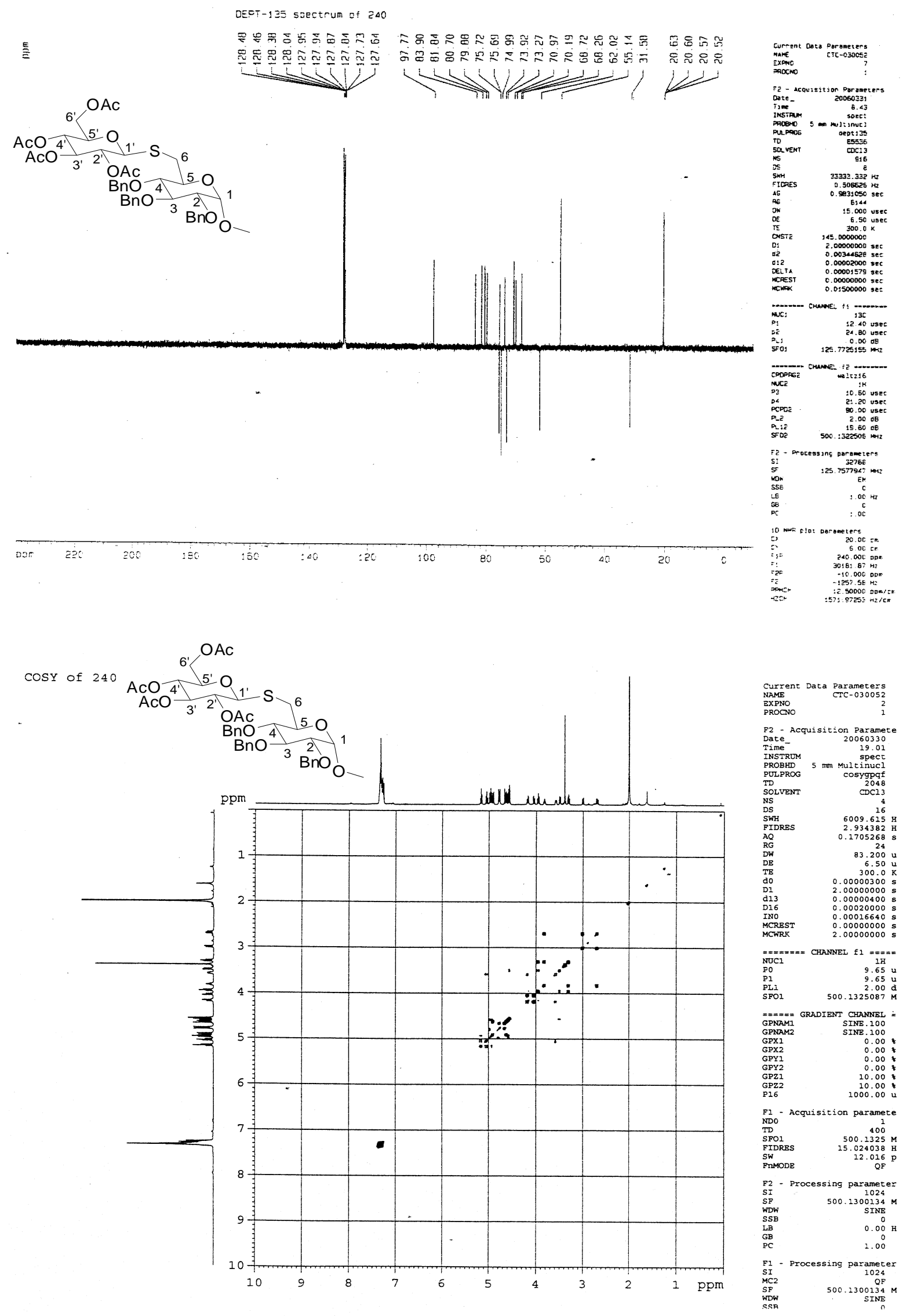

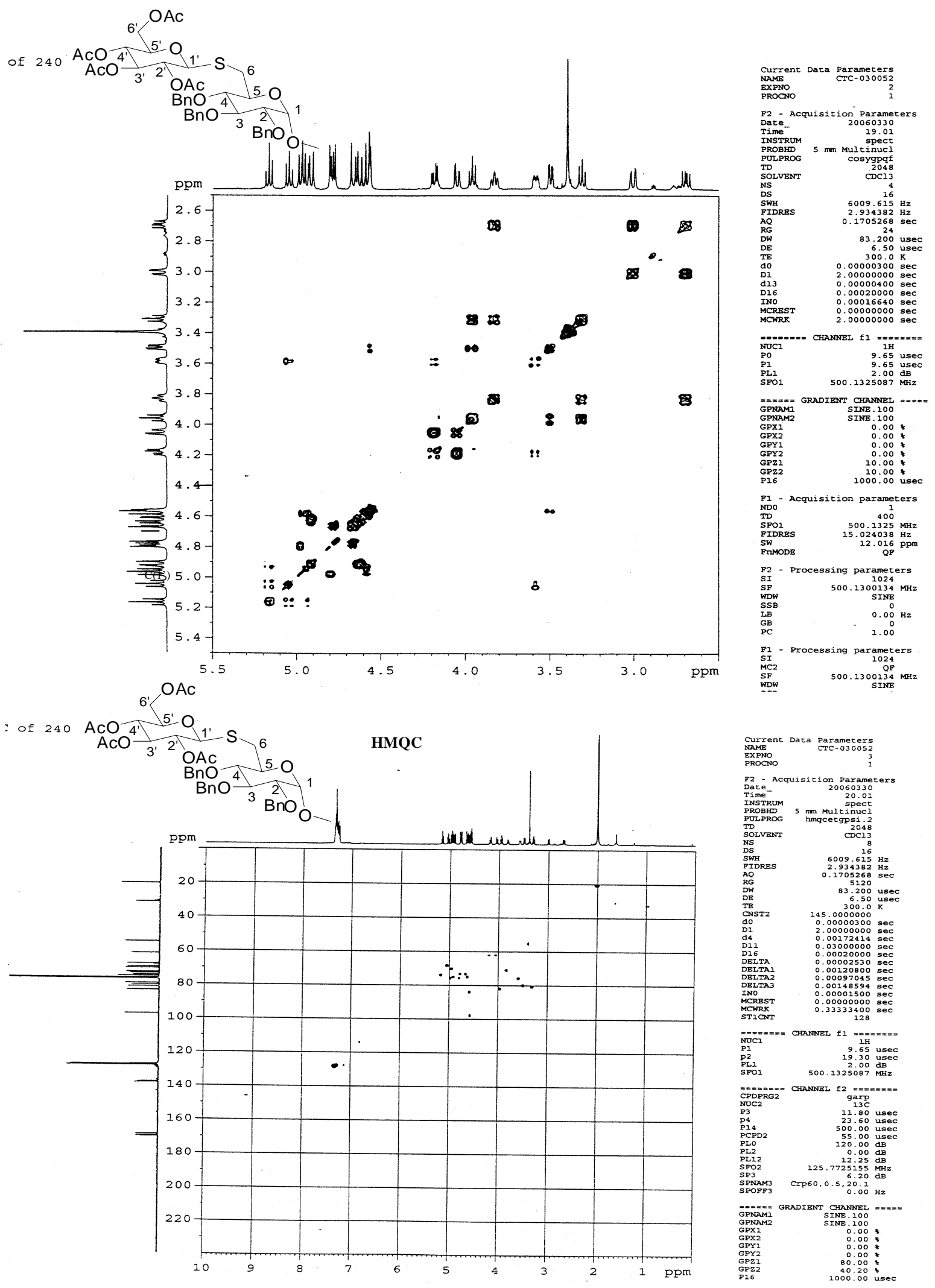

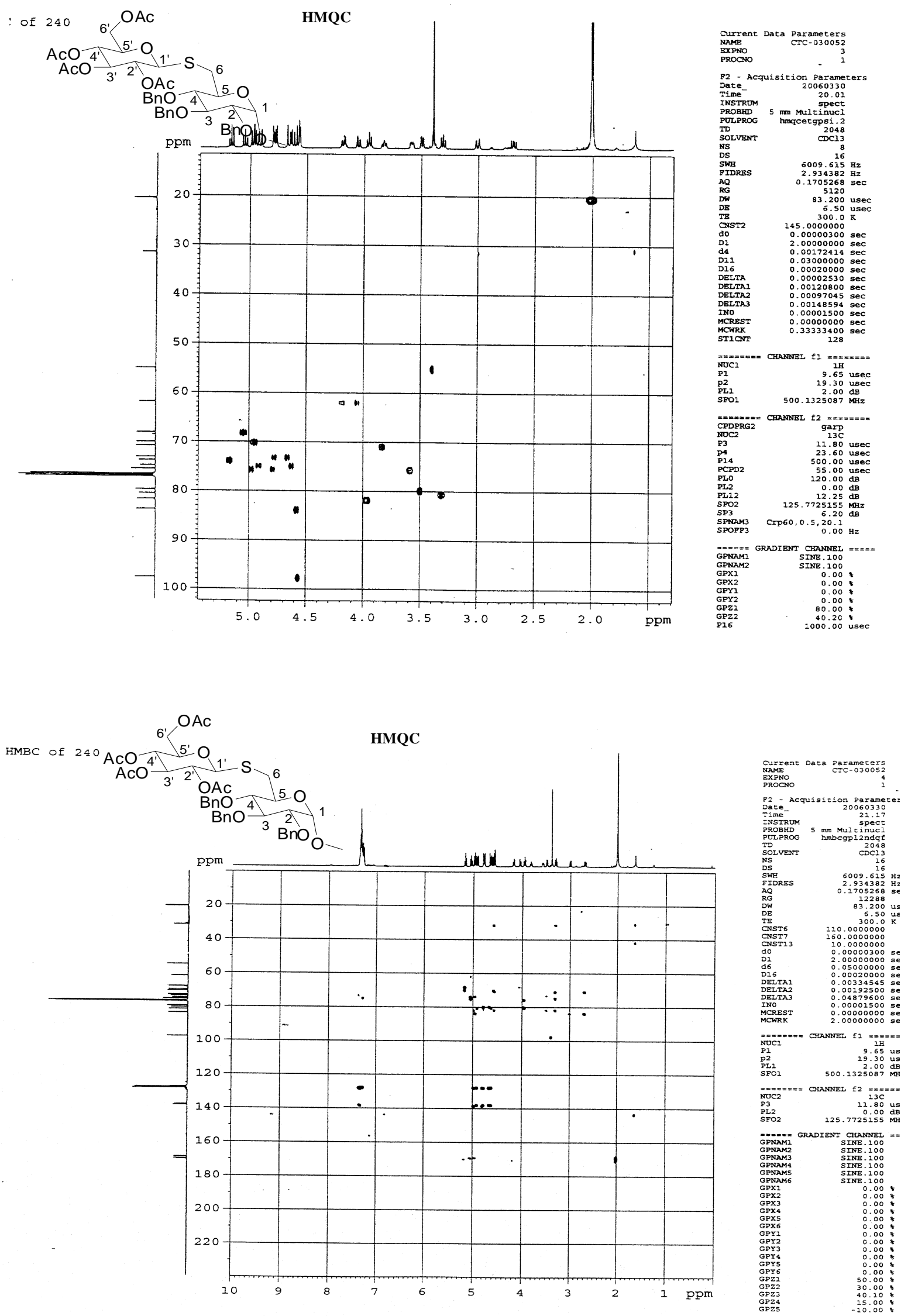